\title{
14. PETROLOGIC AND STABLE ISOTOPE CONSTRAINTS ON HYDROTHERMAL ALTERATION AND SERPENTINIZATION OF THE EPR SHALLOW MANTLE AT HESS DEEP (SITE 895) ${ }^{1}$
}

\author{
Gretchen L. Früh-Green, ${ }^{2}$ Alessio Plas, ${ }^{2}$ and Christophe Lécuyer ${ }^{3}$
}

\begin{abstract}
Leg 147 of the Ocean Drilling Program recovered sections of the East Pacific Rise lower crust and shallow mantle $(\approx 1 \mathrm{Ma})$, tectonically exposed at the western end of the Cocos-Nazca propagator of the Hess Deep Rift Valley. These rocks record a polyphase history of hydrothermal alteration and provide new constraints on the depth and mechanisms of hydrothermal circulation at fast-spreading ridges. A complex sequence of harzburgite-dunite-troctolite-gabbro recovered at Site 895 is considered to be the result of processes of melt migration and wall-rock reaction close to the mantle/crust boundary. The peridotites are extensively serpentinized $(50 \%-100 \%)$ and are cut by multiple generations of fracture-filling veins. In the gabbros, progressive alteration under greenschist to zeolite facies conditions is characterized by tremolite + chlorite + diopside + anorthite \pm prehnite assemblages in the least altered samples, and incipient rodingitization to prehnite + hydrogrossular + zeolite + clays as cataclastic deformation and veining increases.

Oxygen isotope ratios of mineral separates from the gabbros and peridotites from Site 895 show a depletion in ${ }^{18} \mathrm{O}$ relative to mantle values and are consistent with high-temperature exchange with aqueous fluids. Dunite/harzburgite ratios of chlorite, serpentine, and tremolite, together with $\delta^{13} \mathrm{C}$ values of $\mathrm{CO}_{2}$ extracted from completely serpentinized dunites, suggest at least two, but possibly three, components of the hydrothermal fluids: hydrothermally altered seawater; magmatic volatiles; and $\mathrm{H}_{2}$ released during serpentinization. These data combined with structural data imply that penetration of seawater and high-temperature hydrothermal alteration produced a low ${ }^{18} \mathrm{O}$ shallow mantle sequence at some distance off-axis of the East Pacific Rise, but at an early stage in the propagation of the Cocos-Nazca rift and formation of the Hess Deep Rift Valley. Mineral assemblages in the gabbroic rocks and the presence of antigorite at Hess Deep, combined with oxygen isotope ratios, suggest that faulting associated with the Cocos-Nazca propagator enhanced seawater penetration and hydrothermal alteration at temperatures above $350^{\circ} \mathrm{C}$ in this segment of the East Pacific Rise oceanic lithosphere. The results of this study suggest that seawaterperidotite interactions and high-temperature serpentinization processes may be an important contribution to the overall ${ }^{18} \mathrm{O}$ budget in the oceanic lithosphere nd may represent a significant sink for mantle $\mathrm{CO}_{2}$ and source of $\mathrm{H}_{2}$.
\end{abstract}

\section{INTRODUCTION}

In recent years, numerous studies of altered oceanic rocks and hydrothermal vent fluids have provided clear evidence that submarine hydrothermal systems play an important role in crustal evolution at mid-ocean ridge systems. In addition to the importance of fluid circulation in the transport of heat from young oceanic crust to the oceans, seawater-rock reactions control hydrothermal vent fluid chemistries and play a major role in buffering the chemical and isotopic compositions of seawater (e.g., Muehlenbachs and Clayton, 1976; Von Damm, et al., 1985; Alt et al., 1986b; Thompson, 1983). However, the depth to which seawater penetrates into the oceanic lithosphere and the controls on hydrothermal circulation in the mantle are still poorly understood. Hydration of the oceanic mantle has major consequences for geophysical properties in that serpentinization is accompanied by a decrease in bulk density that directly affects the physical properties of the mantle, gravity signatures, and seismic velocities. The formation of magnetite during the hydration process may also play a crucial role in contributing to marine magnetic anomalies as well as providing a source of hydrogen and methane to hydrothermal fluids. It is thus important to understand the controls on seawater penetration into the mantle and to determine the active or passive role of serpentinization; that is whether extensional structures are required for serpentinization processes to occur or whether ser-

'Mével, C., Gillis, K.M., Allan, J.F., and Meyer, P.S. (Eds.), 1996. Proc. ODP, Sci, Results, 147: College Station, TX (Ocean Drilling Program).

${ }^{2}$ Department of Earth Sciences, Eidgenössische Technische Hochschule-Zürich (ETH-Zurich), CH-8092 Zürich, Switzerland. gretli@erdw.ethz.ch

'Laboratoire de Géochemie Isotopique, Géosciences Rennes, CNRS UPR4661, Campus de Beaulieu, Université de Rennes 1, F-35042 Rennes, France. pentinite protrusions and diapirism are required for structures to form (e.g., Bonatti, 1976; Boillot et al., 1980; Francheteau et al., 1990). Fundamental to understanding the processes of serpentinization is the determination of the depth and temperature of seawater penetration, which in turn may shed light on the relative timing of serpentinization in the overall tectonic history of ocean basins. Stable isotope geochemistry, in particular, can be applied to monitor fluid-related processes in order to determine fluid sources and scales of fluid-rock interaction and the relationship to deformation. In addition, the isotopic signatures of modern oceanic rocks also allow the extent and temperatures of seawater circulation and the possible interaction between high-temperature metamorphism and late-stage magmatic processes to be inferred.

\section{Geologic and Tectonic Overview}

The Hess Deep Rift Valley is a tectonically complex, westwardpropagating oceanic rift valley located near the junction of the Pacific, Nazca, and Cocos plates west of the Galapagos Islands (Fig. 1). Hess Deep, the deepest part ( $>5400$ meters below seafloor [mbsf]) of this rift valley, is the result of deep faulting associated with the westward propagation of the east-west trending Cocos-Nazca spreading center that is opening up the eastern flank of young oceanic crust (0.5-1 Ma) created at the fast-spreading equatorial East Pacific Rise (EPR) (Lonsdale, 1988; Francheteau et al., 1990). A major, faultbounded, intra-rift ridge, approximately $8 \mathrm{~km}$ wide and $25 \mathrm{~km}$ long, with a flattened crest and an oval dome shape has formed between the Hess Deep and the northern scarp that bounds the valley. The intrarift ridge is oriented east-west, perpendicular to the EPR, and overlaps the western end of the Cocos-Nazca spreading center. The scarps that bound the Hess Deep Rift Valley and the intra-rift ridge were investigated during a Nautile dive program in 1988 (Francheteau et al., 


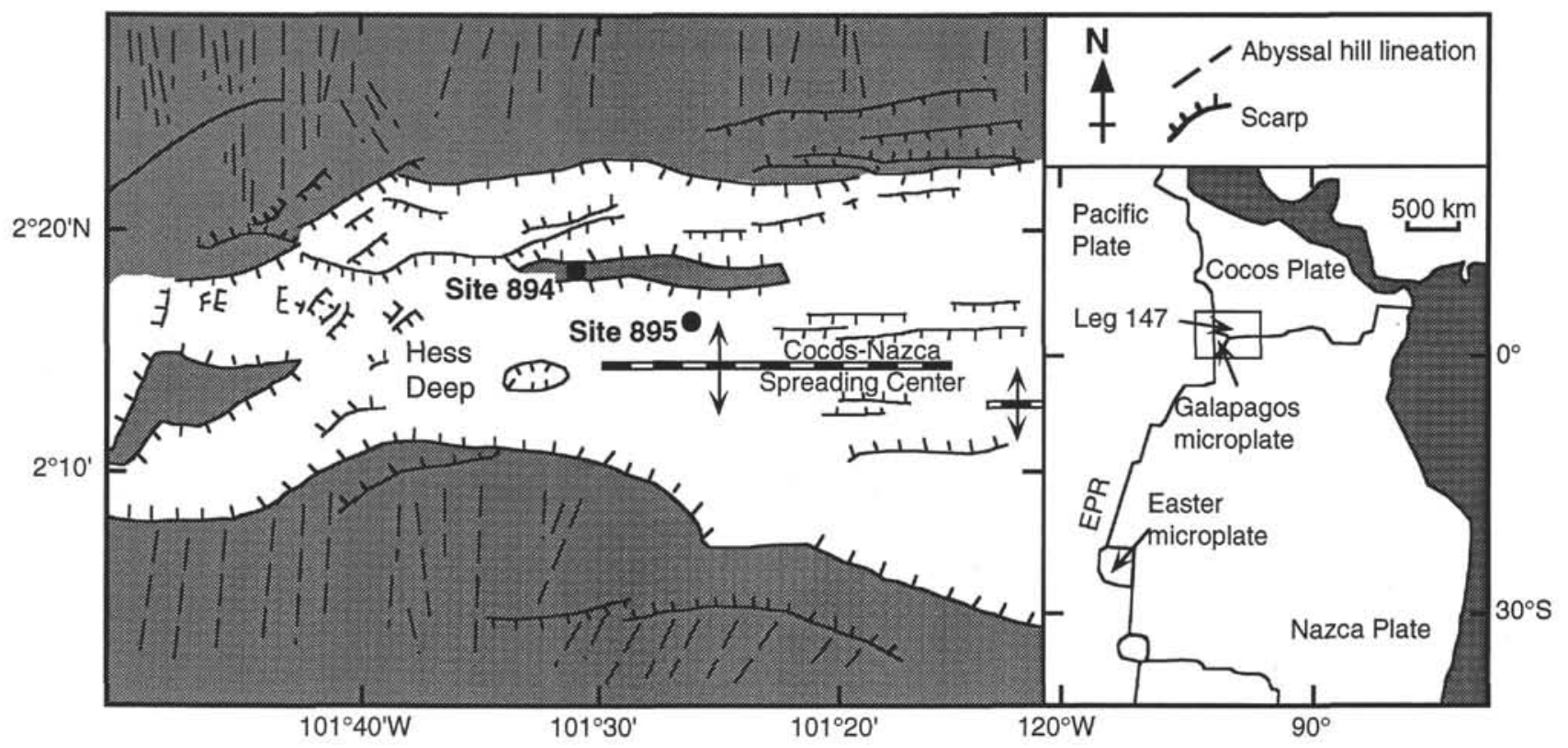

Figure 1. Tectonic location of Hess Deep at the western end of the Cocos-Nazca spreading center. After Lonsdale (1988) and Karson et al. (1992).

1990; Francheteau et al., 1992) and an Alvin dive program in 1989 (Karson et al., 1992). The results of these dive programs showed that a complete, relatively fresh, crustal section (consisting of volcanics, sheeted dikes, and minor gabbros) is exposed along the scarps that bound the rift valley. Similar; but more dismembered, sections of the EPR oceanic crust (including lavas, dikes, gabbros, and peridotites) are exposed on the floor of the Hess Deep Rift Valley and along the intra-rift ridge (Francheteau et al., 1990; Hekinian et al., 1993).

Two alternative rifting models have been proposed for the formation of the Hess Deep Rift Valley and the emplacement of deep crustal rocks at shallow levels in the oceanic crust (see fig. 6 in Francheteau et al., 1990). The first model places emphasis on the overall symmetry of the rift valley and suggests that the structure is a result of vertical movements during isostatic readjustment and diapiric uplift of serpentinized mantle. The second model invokes a mechanism of low-angle detachment to expose and entrain upper mantle and lower crustal rocks. This latter model implies that extension was accommodated by rotation of large crustal blocks along listric normal faults similar to those in rifted continental lithosphere and recently postulated for slow-spreading ridges and the Galicia margin (Karson et al., 1987; Boillot et al., 1980).

Leg 147 took advantage of the tectonic exposure at Hess Deep and successfully cored two sites, recovering continuous sections of $\approx 1$ Ma lower crust and upper mantle that were created at the EPR. The first site, Site $894\left(2^{\circ} 18^{\prime} \mathrm{N}, 101^{\circ} 31.5^{\prime} \mathrm{W}\right)$, is located near the summit at the western end of the intra-rift ridge in water depths between 3023 and $3044 \mathrm{~m}$, close to the site where gabbroic rocks were recovered during the 1988 Nautile dive campaign. The second site, Site 895 $\left(2^{\circ} 16.7^{\prime} \mathrm{N}, 101^{\circ} 26.7^{\prime} \mathrm{W}\right)$, is located $9 \mathrm{~km}$ southeast of Site 894 , along the southern slope of the intra-rift ridge in an area where the Nautile recovered peridotites interpreted to represent the EPR shallow mantle. Six holes ( $895 \mathrm{~A}$ to $895 \mathrm{~F}$ ) were drilled at three different locations at depths between 3820 and $3692 \mathrm{~m}$ and penetrated a total of 272.9 $\mathrm{m}$, recovering $64.56 \mathrm{~m}$ of upper mantle rocks with an average recovery of $27.5 \%$. Substantial penetration was obtained only in Holes $895 \mathrm{C}(37.9 \mathrm{~m}), 895 \mathrm{D}(93.7 \mathrm{~m})$ and $895 \mathrm{E}(87.6 \mathrm{~m})$, with a total recovery of $5.7 \mathrm{~m}, 20.0 \mathrm{~m}$, and $32.9 \mathrm{~m}$, respectively. Serpentinized harzburgites and dunites, the most abundant rock type recovered, were intercalated with troctolites, olivine gabbros, gabbros, and minor basaltic dikes. The proportion of the four major rock types varied among the holes that had significant penetration and may indicate that the shallow mantle below the EPR is laterally heterogeneous on a scale of tens to hundreds of meters. The association of harzburgitedunite-gabbro, similar to the transition zone in many ophiolite complexes, as well as the relative abundance of dunite, suggests that Site 895 was drilled close to the mantle/crust boundary (Gillis, Mével, Allan, et al., 1993; Shipboard Scientific Party, 1993a, 1993b).

Paleomagnetic measurements from Hole 895D show widely scattered stable magnetic inclination, suggesting that the cores may have penetrated several large blocks that have been rotated to different extents. In contrast, the relatively constant stable magnetic inclinations from Hole $895 \mathrm{E}$ are similar to those obtained from gabbros at Hole $894 \mathrm{G}$. These data suggest that the tectonic rotation took place after the major serpentinization event (Gillis, Mével, Allan, et al., 1993; Shipboard Scientific Party, 1993a, 1993b; Pariso et al., this volume; Kelso et al., this volume).

In this study, petrologic and stable isotope data are presented to characterize the hydrothermal alteration and deformation history of the shallow mantle sequence at Site 895 and to document the extent, mechanisms, and relative timing of seawater penetration into oceanic lithosphere at Hess Deep. The study of alteration and serpentinization of mantle peridotites provides information as to the role of serpentinization processes in the overall tectonic evolution of the Hess Deep area. Furthermore, the relative timing of the gabbroic intrusions and their alteration may have had important consequences on the degree to which magmatic volatiles contributed to the isotopic and chemical composition of circulating hydrothermal fluids.

\section{ANALYTICAL METHODS}

A set of 59 polished thin sections from Holes $895 \mathrm{C}, 895 \mathrm{D}$, and $895 \mathrm{E}$ was used for petrographic descriptions and electron microprobe analyses. Mineral chemistries were determined using a Cameca SX50 electron microprobe with natural minerals for standards and a standard correction program for oxides and silicates. Routine operating conditions were $15 \mathrm{kV}$ accelerating voltage, $20 \mathrm{nA}$ sample current, $20-30$ s/element counting time and a $1-10 \mu \mathrm{m}$ spot size. Analyses of hydrogen, oxygen, and carbon isotopes were carried out on mineral separates and vein material. Mineral separates were obtained 
from crushed, sieved, and ultrasonically cleaned samples, using a Frantz magnetic separator and standard heavy liquid techniques. The mineral separates were further rinsed in $3 \mathrm{M} \mathrm{HCl}$ to remove carbonates and surface contamination and rinsed in distilled water before hand picking. Aragonite samples were obtained directly from veins using a hand drill, then crushed in an agate mortar. Purity of the separates (in most cases better than 95\%) and fine-grained alteration phases were determined by X-ray diffraction (XRD) analysis on a Scintag X-ray diffractometer. Detailed XRD analyses were also made on representative rock- and vein-serpentine separates to better characterize the serpentine minerals and to determine which of the three common serpentine-group minerals is most prevalent. Particular attention was made to eliminate crosscutting veins and veinlets from rock-serpentine separates. Nevertheless, minor amounts of bastite-derived serpentine and very thin (less than $0.5 \mathrm{~mm}$ ) veinlet-serpentine may be included in the separates. The samples were run between $2^{\circ}$ and $65^{\circ} 2 \theta, 40 \mathrm{kV} / 20 \mathrm{~mA}$, using $\mathrm{CuK} \alpha$ radiation, and a scan speed of $2 \% \mathrm{~min}$, with quartz as an internal standard.

Carbon and oxygen isotope ratios from a set of ten aragonite veins were determined on $\mathrm{CO}_{2}$ gas liberated by reaction with phosphoric acid (McCrea, 1950). Oxygen was liberated from silicate samples by reaction with $\mathrm{ClF}_{3}$ at $600^{\circ} \mathrm{C}$ (Borthwick and Harmon, 1982) and converted to $\mathrm{CO}_{2}$ by reaction with heated carbon. For hydrogen isotope analysis, hydrous mineral separates were dried at $150^{\circ} \mathrm{C}$ under vacuum overnight, then heated in a vacuum to $>1100^{\circ} \mathrm{C}$ to liberate $\mathrm{H}_{2}$ and $\mathrm{H}_{2} \mathrm{O}$. Molecular hydrogen was converted to water by reaction with copper oxide. The resulting total water was quantitatively converted to hydrogen by reaction with zinc. The isotopic ratios of mineral separates and vein phases were determined by conventional mass spectrometric analysis on VG OPTIMA and PRISM dual-inlet mass spectrometers at the Eidgenössische Technische Hochschule-Zürich (ETH), calibrated against Vienna-Standard Mean Ocean Water (VSMOW), Standard Light Antarctic Precipitation (SLAP), and Greenland Ice Sheet Precipitation (GISP). Oxygen isotope analyses of magnetite separates were carried out using laser-based microanalytical techniques (Sharp, 1990) at the University of Lausanne, Switzerland.

$\mathrm{CO}_{2}$ for carbon isotope analysis was extracted from completely serpentinized harzburgite Sample 147-895E-8R-2 (Piece 7, 73-77 $\mathrm{cm}$ ) and dunite Sample 147-895E-2R-2 (Piece 9, 62-65 cm) by thermal decrepitation. Samples weighing from 300 to $400 \mathrm{mg}$ were degassed at $70^{\circ}-80^{\circ} \mathrm{C}$ for a minimum of three hours under vacuum. $\mathrm{CO}_{2}$ was extracted from the dunite sample one time at a temperature of $600^{\circ} \mathrm{C}$ and twice at a temperature of $800^{\circ} \mathrm{C}$. The harzburgite sample was heated one time to temperatures of $600^{\circ}$ and $1000^{\circ} \mathrm{C}$, and twice to a temperature of $800^{\circ} \mathrm{C}$. To evaluate the possible effect of trace amounts of carbonate on the carbon isotope compositions of $\mathrm{CO}_{2}$ liberated by the two peridotite samples and to compare isotopic ratios of $\mathrm{CO}_{2}$-derived marine carbonates, an aragonite vein taken from Sample 147-895E-3R (Piece 9, 96-98 cm) was also heated at temperatures of $600^{\circ}$ and $800^{\circ} \mathrm{C}$. Heating rates were set at $30^{\circ}-35^{\circ} \mathrm{C} / \mathrm{min} . \mathrm{CO}_{2}$ was separated cryogenically and its volume measured with a manometer to a precision of $5 \mu \mathrm{mol}$. The liberated $\mathrm{CO}_{2}$ was analyzed on a VG SIRA 10 mass spectrometer at the University of Rennes I.

All isotope values are reported in standard $\delta$-notation in per mil (\%o) relative to the Pee Dee Belemnite (PDB) isotopic standard for carbon and relative to V-SMOW for oxygen and hydrogen. During the time period from June 1993 to June 1994 in the ETH laboratory, the international quartz standard NBS- 28 gave a value of $9.57 \%$ with a $1 \sigma$ standard deviation of $0.25 \%$. An internal laboratory standard, calibrated against NBS- 30 with a value of $-65 \%$, was used for hydrogen isotope analyses. Repeat measurements of individual samples yielded an overall reproducibility of $\pm 0.2 \%$ for oxygen isotope ratios and $\pm 1 \%$ for hydrogen isotope ratios. The overall reproducibility for carbon isotope ratios was $\pm 0.1 \%$.

\section{PETROLOGY AND MINERAL CHEMISTRY}

The following section describes the petrology and metamorphic evolution of the ultramafic and gabbroic rocks recovered in Holes $895 \mathrm{C}, 895 \mathrm{D}$, and $895 \mathrm{E}$. A summary of mineral modes, degree of alteration and deformation characteristics of the investigated samples are given in Table 1. Due to the small sample size and the heterogeneous nature of alteration, only ranges in modal compositions are presented. These data provide evidence for a complex hydrothermal history involving multiple phases of deformation, fluid infiltration, and veining under varying thermal and tectonic conditions.

\section{Ultramafic Lithologies}

The ultramafic rocks recovered at Site 895 consist of variably altered porphyroclastic harzburgites and dunites, with less-abundant plagioclase-impregnated dunites that locally become more mafic and reach troctolitic compositions (Fig. 2). The dunites and troctolitic rocks are invariably associated with intervals in which gabbroic lithologies are more abundant. Although Hole $895 \mathrm{C}$ consists primarily of porphyroclastic harzburgite, a striking gradational sequence from harzburgite through dunite to troctolite and olivine gabbro is observed in Core 147-895C-4R. The transition from ultramafic to mafic lithologies is particularly well preserved in Sections 147 $895 \mathrm{C}-4 \mathrm{R}-1$ through $4 \mathrm{R}-3$, and is marked by a gradual increase in modal proportions of interstitial plagioclase and clinopyroxene as impregnations in the dunites.

A considerable difference in the proportion of the major lithologies is observed between Holes $895 \mathrm{D}$ and $895 \mathrm{E}$, with a predominance of harzburgite in Hole $895 \mathrm{D}$ and an abundance of dunite and gabbroic rocks in Hole 895E. In the upper part of Hole 895D (from Cores 147$895 \mathrm{D}-1 \mathrm{R}$ to $6 \mathrm{R}$ ), the dominant lithology is pervasively altered, greenish to dark gray, weakly porphyroclastic harzburgite with minor intervals of dunitic rocks; whereas in Cores 147-895D-8R and 9R the abundance of dunites become proportionally greater than harzburgite. The average degree of alteration, characterized by the serpentinization of olivine, ranges from $50 \%$ to $100 \%$ in the harzburgites and from $80 \%$ to $100 \%$ in dunites. In Hole $895 \mathrm{E}$ the ultramafic igneous rocks mainly consist of extensively to totally altered, mesh-textured dunites with minor amounts of highly $(90 \%-100 \%)$ serpentinized harzburgites.

\section{Alteration of Ultramafic Rocks}

The primary assemblage of the Site 895 dunitic and harzburgitic rocks consists of olivine, enstatite, augite, spinel, and trace amounts of plagioclase. In the harzburgites, the primary clinopyroxene content is usually lower than 1 modal $\%$, but may locally reach $2 \%-3 \%$. The modal proportion of relict primary phases never exceeds $40 \%$ $50 \%$ in the harzburgites and $20 \%$ in the dunites (Table 1). Replacement is dominated by the serpentinization of olivine. In addition, serpentine pseudomorphs (commonly called bastite) usually replace primary enstatite grains.

\section{Olivine}

Olivine is the most abundant primary phase in the ultramafic rocks and commonly exhibits highly variable and multiple phases of alteration. In the least-altered harzburgites, fresh olivine occurs as individual, small (from a few $\mu \mathrm{m}$ to $2 \mathrm{~mm}$ ), subrounded grains with thin rims $(<0.1 \mathrm{~mm})$ of mesh-textured serpentine along the grain boundaries, or as clusters of unaltered, optically continuous kernels $(0.1-0.3 \mathrm{~mm})$, surrounded by an anastomosing network of veinlets composed of antigorite(?) \pm chlorite \pm clay, with secondary magnetite in the center of the veinlets. The veinlets represent early microfrac- 


\begin{tabular}{|c|c|c|c|c|c|c|c|c|c|c|c|c|c|c|c|c|c|c|c|c|c|c|c|c|c|}
\hline \multirow[b]{2}{*}{$\begin{array}{l}\text { Core, section, } \\
\text { interval (cm) }\end{array}$} & \multirow[b]{2}{*}{ Piece } & \multirow[b]{2}{*}{$\begin{array}{l}\text { Depth } \\
\text { (mbsf) }\end{array}$} & \multirow[b]{2}{*}{ Lith. } & \multirow[b]{2}{*}{$\begin{array}{l}\text { Alteration } \\
(\%)\end{array}$} & \multicolumn{5}{|c|}{ Relict primary phases } & \multicolumn{14}{|c|}{ Secondary phases } & \multirow[b]{2}{*}{ Veins } & \multirow[b]{2}{*}{ Texture/fabric } \\
\hline & & & & & olv & opx & $\mathrm{cpx}$ & plg & sp/ox & tr & scpx & spl & chl & serp & $\mathrm{tlc}$ & br & $\mathrm{mt} / \mathrm{ox}$ & pre & hgr & cly & zeo & cz/ep cc/arg & hox & & \\
\hline $\begin{array}{l}147-895 \mathrm{C}- \\
1 \mathrm{R}-1,32-35\end{array}$ & 5 & 0.32 & $\mathrm{HZ}$ & $70-90$ & $\mathrm{OO}$ & $\mathrm{T}$ & $\mathrm{T}$ & & $\mathrm{T}$ & T & $\mathrm{T}$ & & $\mathrm{T}$ & $\mathrm{xX}$ & $\mathrm{T}$ & & $\mathrm{OO}$ & & & $\mathrm{OO}$ & & & & V/MV: serp $+\mathrm{mt} \pm \mathrm{chl}$ & porph vnd \\
\hline $3 R-1,90-93$ & 15 & 19.2 & $\mathrm{HZ}$ & $80-100$ & 0 & $\mathrm{~T}$ & $\mathrm{~T}$ & & $\mathrm{~T}$ & 0 & $\mathrm{~T}$ & & o & $\mathrm{xx}$ & & $\mathrm{T}$ & $\mathrm{OO}$ & & & 0 & & & & $\begin{array}{l} \pm \mathrm{dc} \\
\mathrm{V} / \mathrm{MV}: \mathrm{serp}+\mathrm{mt} \text { serp }+ \\
\mathrm{mt} \pm \mathrm{br}\end{array}$ & porph svnd \\
\hline $4 \mathrm{R}-1,52-54$ & ID & 28.42 & IDU & $60-100$ & M & & 0 & & & oO & $\mathrm{o}$ & & $\mathrm{x}$ & $\mathrm{x}$ & & $\mathrm{T}$ & 0 & & & $\mathrm{~T}$ & & o & & $\begin{array}{l}\text { V: serp }+ \text { chl } \pm \mathrm{mt} \text {; chl } \\
\quad+\mathrm{tr}\end{array}$ & svnd \\
\hline $4 \mathrm{R}-1,54-57$ & IE & 28.44 & IMDU & $90-100$ & $\mathrm{OO}$ & & & $\mathrm{T}$ & $\mathrm{T}$ & OO & $\mathrm{o}$ & & M & M & $\mathrm{T}$ & & $\mathrm{o}$ & & $\mathrm{T}$ & $\mathrm{OO}$ & & & & $\begin{array}{l}\mathrm{MG} / \mathrm{MV} \text { : serp, chl, cly, } \\
\mathrm{mt}, \mathrm{cc}\end{array}$ & $\bmod$ fr \\
\hline $\begin{array}{l}4 R-2,40-45 \\
4 R-3,13-16\end{array}$ & $\begin{array}{c}7 \\
2 \mathrm{~A}\end{array}$ & $\begin{array}{l}29.38 \\
30.61\end{array}$ & $\begin{array}{l}\text { MGB } \\
\text { IDU }\end{array}$ & $\begin{array}{c}100 \\
70-90\end{array}$ & $\begin{array}{l}\mathrm{T} \\
\mathrm{M}\end{array}$ & & $\mathrm{T}$ & $\mathrm{T}$ & $\mathrm{T}$ & $\begin{array}{l}\mathrm{X} \\
\mathrm{T}\end{array}$ & $\underset{\mathrm{T}}{\mathrm{OO}}$ & & $\begin{array}{c}\mathrm{X} \\
\mathrm{OO}\end{array}$ & $\stackrel{\mathrm{OO}}{\mathrm{x}}$ & $?$ & $\mathrm{~T}$ & $\begin{array}{c}\mathrm{T} \\
\mathrm{OO}\end{array}$ & & $\mathrm{T}$ & $\mathrm{T}$ & & $\mathrm{T}$ & & $\begin{array}{l}\text { V: serp + dio /MV: arg } \\
\text { V: serp + chl + mt/MV: } \\
\text { serp + mt + br }\end{array}$ & shrd; def veins; cont spt \\
\hline \multicolumn{26}{|l|}{ 147-895D- } \\
\hline $2 \mathrm{R}-2,29-32$ & 2 & 17.71 & $\mathrm{HZ}$ & $70-90$ & $\mathrm{OO}$ & $\mathrm{O}$ & $\mathrm{T}$ & & $\mathrm{T}$ & $\mathrm{T}$ & T & & $\mathrm{T}$ & $\mathrm{XX}$ & $\mathrm{T}$ & & o & & & $\mathrm{T}$ & & & & MV: $t c ;$ serp + mt & porph mesh txt \\
\hline $3 R-1,123-126$ & 15 & 27.23 & $\mathrm{HZ}$ & $65-95$ & $\mathrm{OO}$ & & $\mathrm{T}$ & & $\mathrm{T}$ & $\mathrm{T}$ & $\mathrm{T}$ & & $\mathrm{T}$ & $\mathrm{xx}$ & & $\mathrm{T}$ & 0 & & & 0 & & & & $\begin{array}{l}\text { V: } \operatorname{serp}+m t / M V: \\
\text { serp }+b r+m t\end{array}$ & porph vnd \\
\hline $4 \mathrm{R}-2,45-49$ & $7 \mathrm{~B}$ & 36.04 & DU & $80-100$ & OO & $T$ & $T$ & & $\mathrm{~T}$ & $T$ & $\mathrm{~T}$ & & $\mathrm{O}$ & $\mathrm{XX}$ & & $\mathrm{T}$ & o & & & o & & & & V/MV: serp + mt + chl & vnd mesh $\mathrm{txt}$ \\
\hline $4 R-2,126-129$ & 18 & 36.79 & $\mathrm{HZ}$ & $50-90$ & M & $\mathrm{T}$ & $\mathrm{T}$ & & $\mathrm{T}$ & $\mathrm{T}$ & $T$ & & $T$ & & & & o & & & o & & & & $\mathrm{V} / \mathrm{MV}:$ serp $+\mathrm{mt}$ & porph \\
\hline $4 \mathrm{R}-4,5-9$ & $2 \mathrm{~A}$ & 3.5 & $\mathrm{HZ}$ & $50-80$ & $\mathrm{M}$ & OO & $\mathrm{T}$ & & 0 & $\mathrm{~T}$ & $\mathrm{~T}$ & & $\mathrm{~T}$ & $\mathrm{xx}$ & $\mathrm{T}$ & & $\mathrm{o}$ & & & $\mathrm{O}$ & & & & $\begin{array}{l}\text { V/MV: serp }+\mathrm{mt}+\mathrm{chl} / \\
\text { MV: } \mathrm{tc}\end{array}$ & porph vnd \\
\hline $4 \mathrm{R}-4,39-43$ & 5 & 38.84 & $\mathrm{HZ}$ & $85-100$ & $\mathrm{OO}$ & $\mathrm{T}$ & $\mathrm{T}$ & & $\mathrm{T}$ & $\mathrm{T}$ & $\mathrm{T}$ & & $\mathrm{T}$ & $\mathrm{XX}$ & $\mathrm{T}$ & $\mathrm{T}$ & M & & & 0 & & $\mathrm{~T}$ & & MV: serp $+m t \pm b r$ & porph mesh txt \\
\hline $4 R-4,96-99$ & 14 & 39.41 & $\mathrm{HZ}$ & $95-100$ & $\mathrm{~T}$ & $\mathrm{~T}$ & $\mathrm{~T}$ & & $\mathrm{~T}$ & $\mathrm{~T}$ & $\mathrm{~T}$ & & $\mathrm{~T}$ & $\mathrm{xx}$ & $\mathrm{T}$ & & 0 & & & $\mathrm{~T}$ & & $\mathrm{~T}$ & & $\begin{array}{l}\mathrm{V}: \arg / \mathrm{MV}: \text { serp }+\mathrm{mt} \\
\mathrm{tc}\end{array}$ & porph vnd \\
\hline $5 \mathrm{R}-2,41-49$ & 8 & 43.67 & $\mathrm{HZ}$ & $60-70$ & M & & & & $\mathrm{T}$ & $\mathrm{T}$ & $\mathrm{T}$ & & $\mathrm{T}$ & $\mathrm{XX}$ & & & o & & & $\mathrm{O}$ & & & & $\mathrm{V}: \mathrm{serp}+\mathrm{mt}$ & svnd mesh txt \\
\hline $5 R-2,44-51$ & 7 & 45.18 & $\mathrm{HZ}$ & $75-95$ & OO & 0 & $\mathrm{~T}$ & & $\mathrm{~T}$ & $\mathrm{~T}$ & $\mathrm{~T}$ & & $\mathrm{~T}$ & $\mathrm{xX}$ & $\mathrm{T}$ & & 0 & & & 0 & & & & $\begin{array}{l}\text { V: serp (asb) / MV: serp } \\
+\mathrm{mt}\end{array}$ & porph mesh txt \\
\hline $5 R-2,107-108$ & 14 & 45.81 & $\mathrm{HZ}$ & $80-100$ & $\mathrm{OO}$ & 0 & $\mathrm{~T}$ & & $\mathrm{~T}$ & $\mathrm{~T}$ & $\mathrm{~T}$ & & $\mathrm{~T}$ & $\mathrm{xx}$ & $\mathrm{T}$ & & 0 & & & $\mathrm{OO}$ & & 0 & & $\begin{array}{l}\mathrm{V}: \arg / \mathrm{MV}: \text { serp + mt; } \\
\text { tc }\end{array}$ & vnd mesh txt \\
\hline $7 \mathrm{R}-1,81-84$ & 13 & 65.41 & TRO & $75-90$ & oo & & & $\mathrm{T}$ & $\mathrm{T}$ & 00 & o & & M & M & $\mathrm{T}$ & & 0 & $\mathrm{~T}$ & o & 0 & & & & $\begin{array}{l}\text { MG/MV: serp; chl; cly; } \\
\text { mt; dio; tr }\end{array}$ & $\bmod f \mathrm{r}$ \\
\hline $\begin{array}{l}8 R-1,52-59 \\
8 R-1,122-126\end{array}$ & $\begin{array}{r}8 \\
19\end{array}$ & $\begin{array}{l}74.82 \\
75.52\end{array}$ & $\begin{array}{c}\text { OLGB } \\
\mathrm{HZ}\end{array}$ & $\begin{array}{c}60-90 \\
95-100\end{array}$ & $\begin{array}{l}\mathrm{O} \\
\mathrm{O}\end{array}$ & $\mathrm{T}$ & $\mathrm{T}$ & M & $\mathrm{T}$ & $\stackrel{\mathrm{M}}{\mathrm{T}}$ & $\mathrm{OO}$ & 0 & $\underset{\mathrm{T}}{\mathrm{OO}}$ & $\mathrm{xX}$ & $\mathrm{T}$ & $\mathrm{T}$ & $\mathrm{OO}$ & 0 & 0 & $\begin{array}{l}\mathrm{O} \\
\mathrm{O}\end{array}$ & $\mathrm{T}$ & $\mathrm{OO}$ & & $\begin{array}{l}\text { V: pre } \\
\text { V: serp }+\mathrm{mt} \pm \text { chl; arg / } \\
\text { MV: serp + br }\end{array}$ & $\begin{array}{l}\text { wk fr } \\
\text { porph svnd }\end{array}$ \\
\hline $8 R-2,14-19$ & 2 & 75.82 & GB & $85-90$ & & & 00 & 0 & & OO & 0 & & M & & & & & M & $\mathrm{o}$ & $\mathrm{o}$ & & & boem & $\begin{array}{l}\text { V: pre + hgr / MV: chl; } \\
\text { cly }\end{array}$ & $\mathrm{dlg} ; \bmod \mathrm{fr} ; \mathrm{kb}$ (cpx) \\
\hline $\begin{array}{l}8 \mathrm{R}-2,32-37 \\
8 \mathrm{R}-2,82-86\end{array}$ & $\stackrel{4}{4}$ & 76 & OLGB & $80-90$ & $\mathrm{OO}$ & & & $\mathrm{O}$ & & OO & $\mathrm{O}$ & & $\mathrm{M}$ & & & & 0 & $\mathrm{M}$ & 0 & $\mathrm{O}$ & 0 & & & MG/MV: chl; tr; cly & $\bmod \mathrm{fr}$ \\
\hline $\begin{array}{l}8 \mathrm{R}-2,82-86 \\
8 \mathrm{R}-2,145-149\end{array}$ & $\begin{array}{l}10 \mathrm{~B} \\
19\end{array}$ & $\begin{array}{l}76.5 \\
77.13\end{array}$ & $\begin{array}{l}\mathrm{HZ} \\
\mathrm{MGB}\end{array}$ & $\begin{array}{c}95-100 \\
100\end{array}$ & $\mathrm{~T}$ & $\mathrm{~T}$ & $\begin{array}{l}\mathrm{T} \\
\mathrm{T}\end{array}$ & & $\begin{array}{l}\mathrm{T} \\
\mathrm{T}\end{array}$ & $\stackrel{T}{x}$ & $\begin{array}{l}\mathrm{T} \\
\mathrm{T}\end{array}$ & & $\begin{array}{l}\mathrm{T} \\
\mathrm{X}\end{array}$ & $\underset{T}{\mathrm{XX}}$ & $\stackrel{T}{T}$ & & 0 & & T? & $\begin{array}{l}\mathrm{T} \\
\mathrm{T}\end{array}$ & & T? & $T$ & $\begin{array}{l}\text { MV: serp + mt; tc } \\
\text { V: serp (atg?) + chl }\end{array}$ & $\begin{array}{l}\text { porph mesh txt } \\
\text { shrd; sb; fol }\end{array}$ \\
\hline $9 \mathrm{R}-1,125-129$ & 19 & 85.25 & DU & 100 & & & & & $\mathrm{~T}$ & & & & $\mathrm{~T}$ & $\mathrm{xx}$ & & $\mathrm{T}$ & $\mathrm{OO}$ & & & 0 & & 0 & & $\begin{array}{l}\text { MV: serp + mt; serp + } \\
\quad \mathrm{br}+\mathrm{mt} / \mathrm{V}: \mathrm{arg}\end{array}$ & vnd mesh txt \\
\hline $\begin{array}{l}9 \mathrm{R}-1,148-150 \\
\mathrm{l}\end{array}$ & 22 & 85.48 & $\mathrm{HZ}$ & 100 & $T$ & & & & $T$ & & & & $\mathrm{~T}$ & $\mathrm{XX}$ & $\mathrm{T}$ & & $\mathrm{O}$ & & & $\mathrm{T}$ & & & & MV: serp + mt & porph mesh txt \\
\hline $\begin{array}{l}\text { 10R-1, 21-25 } \\
\text { 10R-1, 63-70 }\end{array}$ & $\begin{array}{l}3 \\
8\end{array}$ & $\begin{array}{l}93.91 \\
94.33\end{array}$ & $\begin{array}{l}\text { DU } \\
\text { MGB }\end{array}$ & $\begin{array}{l}\text { Up to } 100 \\
100\end{array}$ & $\mathrm{~T}$ & & ${ }_{T}^{T}$ & $\mathrm{~T}$ & T & $\mathrm{x}$ & 0 & & $\begin{array}{l}\mathrm{T} \\
\mathrm{x}\end{array}$ & $\mathrm{xX}$ & & $\mathrm{T}$ & $\mathrm{OO}$ & & & $\mathrm{OO}$ & & $\mathrm{T} ?$ & & MV: serp $+\mathrm{mt} \pm$ br & vnd mesh $\mathrm{xt}$ \\
\hline $147-895 \mathrm{E}-$ & & & & & & & & & & & & & & & & & & & & & & ${ }^{*}$ & & & STIU, 80, Wh TOO \\
\hline IR-1, 1-3 & 1 & 0.01 & MGB & 100 & & & $\mathrm{~T}$ & & & $x \mathrm{x}$ & $\mathrm{T}$ & & $\mathrm{x}$ & $\mathrm{OO}$ & $\mathrm{T}$ & & & & $\mathrm{T}$ ? & $\mathrm{T}$ & & $\mathrm{T}$ ? & $\mathrm{T}$ & $\begin{array}{l}\text { MV: chl } \pm \text { serp; cly; } \\
\text { zeo?; Fe-hox }\end{array}$ & shrd; sb; fol; cont spt \\
\hline $\begin{array}{l}\text { IR-1, 83-87 } \\
\text { IR-1, 105-109 }\end{array}$ & $\begin{array}{r}9 \\
12\end{array}$ & 0.83 & $\begin{array}{l}\text { OLGB } \\
\text { OLGB }\end{array}$ & $\begin{array}{l}35-45 \\
55-65\end{array}$ & $\stackrel{M}{T}$ & & & $\begin{array}{l}\mathrm{x} \\
\mathrm{x}\end{array}$ & T & $\stackrel{\text { OO }}{x}$ & $\begin{array}{l}\text { OO } \\
0\end{array}$ & $T$ & $\begin{array}{l}\mathrm{M} \\
\mathrm{M}\end{array}$ & $\stackrel{T}{T}$ & & & & & & & & & & MV: chl; tr; dio & $\begin{array}{l}\text { local sz } \\
\text { mfre inge sh }\end{array}$ \\
\hline $\begin{array}{l}\text { IR-1, } 105-109 \\
\text { IR-3,71-73 }\end{array}$ & $3 \mathrm{~B}$ & $\begin{array}{l}1.05 \\
3.57\end{array}$ & $\underset{\mathrm{HZ}}{\mathrm{OLGB}}$ & $\begin{array}{l}55-65 \\
95-100\end{array}$ & $\mathrm{~T}$ & $\mathrm{~T}$ & $\mathrm{~T}$ & $\lambda$ & $\mathrm{T}$ & $\begin{array}{l}X \\
T\end{array}$ & 0 & 1 & $\mathrm{O}$ & $\mathrm{xx}$ & $\mathrm{T}$ & $\mathrm{T}$ & 0 & & & $\mathrm{~T}$ & & & & $\mathrm{~V}:$ serp + mt + C /MV: & $\begin{array}{l}\text { mif; incp sh } \\
\text { porph mesh txt }\end{array}$ \\
\hline $2 \mathrm{R}-1,91-95$ & 14 & 20.51 & OLGB & $90-100$ & & & & $\mathrm{OO}$ & & $\mathrm{OO}$ & 0 & 0 & $\mathrm{OO}$ & & & & 0 & M & OO & 0 & o & & 0 & $\begin{array}{l}\text { serp }+\mathrm{mt} \pm \text { tr } \\
\mathrm{v}: \text { pre }+\mathrm{hgr}+\mathrm{Fe} \text {-hox; } \\
\text { zeo }\end{array}$ & fr; local sz/sb \\
\hline $2 \mathrm{R}-1,124-127$ & 18 & 20.84 & OLGB & 85-95 & 0 & & $\mathrm{~T}$ & $\mathrm{OO}$ & $\mathrm{T}$ & M & $\mathrm{o}$ & $\mathrm{T}$ & $\mathrm{x}$ & o & & & $\mathrm{o}$ & o & $\mathrm{o}$ & $\mathrm{T}$ & & & & MV: chl; tr; dio & $\mathrm{mfr} ; \mathrm{msz}$ \\
\hline
\end{tabular}


Table 1 (continued).

\begin{tabular}{|c|c|c|c|c|c|c|c|c|c|c|c|c|c|c|c|c|c|c|c|c|c|c|c|c|c|}
\hline \multirow[b]{2}{*}{$\begin{array}{l}\text { Core, section, } \\
\text { interval }(\mathrm{cm})\end{array}$} & \multirow[b]{2}{*}{ Piece } & \multirow[b]{2}{*}{$\begin{array}{l}\text { Depth } \\
\text { (mbsf) }\end{array}$} & \multirow[b]{2}{*}{ Lith. } & \multirow[b]{2}{*}{$\begin{array}{l}\text { Alteration } \\
(\%)\end{array}$} & \multicolumn{5}{|c|}{ Relict primary phases } & \multicolumn{14}{|c|}{ Secondary phases } & \multirow[b]{2}{*}{ Veins } & \multirow[b]{2}{*}{ Texture/fabric } \\
\hline & & & & & olv & opx & cpx & plg & $\mathrm{sp} / \mathrm{ox}$ & tr & $\operatorname{scpx}$ & $\mathrm{spl}$ & chl & serp & $\mathrm{tc}$ & br & $\mathrm{m} t / \mathrm{ox}$ & pre & hgr & cly & zeo & czlep cc/arg & hox & & \\
\hline $2 R-2,6-10$ & 1 & 21.02 & OLGB & $80-85$ & $\mathrm{~T}$ & & & M & $\mathrm{T}$ & M & $\mathrm{o}$ & T & $\mathrm{x}$ & & & & $\mathrm{T}$ & & & & o & & & V/MV: zeo & mfr; incp sh \\
\hline $2 \mathrm{R}-2,49-51$ & 8 & 21.45 & $\mathrm{DU}$ & 100 & & & & & $\mathrm{~T}$ & $\mathrm{~T}$ & & & $\mathrm{~T}$ & $x \mathrm{x}$ & & $\mathrm{T}$ & $\mathrm{OO}$ & & & $\mathrm{o}$ & & $\mathrm{OO}$ & & $\begin{array}{l}\text { V/MV: serp + mt / V: } \\
\text { arg }\end{array}$ & vnd mesh txt \\
\hline $3 R-1,46-49$ & 8 & 30.06 & DU & 100 & & & & & $\mathrm{~T}$ & & $\mathrm{~T}$ & & $\mathrm{~T}$ & $\mathrm{xx}$ & & $\mathrm{T}$ & $\mathrm{OO}$ & & & $\mathrm{T}$ & & oo & & $\begin{array}{l}\text { V/MV: serp }+\mathrm{mt} \pm \mathrm{br} \pm \\
\mathrm{chl} / \mathrm{V} \text { : arg }\end{array}$ & vnd mesh txt \\
\hline $3 R-2,112-116$ & 11 & 32.09 & DU & 100 & & & & & $\mathrm{~T}$ & & & & $\mathrm{~T}$ & $\mathrm{xX}$ & & $\mathrm{T}$ & $\mathrm{OO}$ & & & $\mathrm{OO}$ & & o & & V/MV: serp + mt/ V: & vnd mesh txt \\
\hline $4 R-2,50-54$ & 4 & 41.44 & IDU & 100 & & & & $\mathrm{~T}$ & $\mathrm{~T}$ & T & & & $\mathrm{T}$ & $x x$ & & $\mathrm{~T}$ & $\mathrm{o}$ & & & OO & & & & $\begin{array}{l}\text { V: serp + mt + chl/ } \\
\text { MV: serp + mt }\end{array}$ & vnd mesh txt \\
\hline 4R-2, 139-141 & 17 & 42.33 & OLGB & $90-100$ & $\mathrm{~T}$ & & $\mathrm{~T}$ & $\mathrm{OO}$ & $\mathrm{T}$ & M & 0 & & M & & & & $\mathrm{T}$ & M & $\mathrm{T}$ & $\mathrm{T}$ & $\mathrm{T}$ & & $\mathrm{T}$ & $\begin{array}{l}\text { V: pre + hgr + Fe-hox, } \\
\text { MV: zeo }\end{array}$ & shrd; sb; mod fol \\
\hline $4 \mathrm{R}-3,29-31$ & 4 & 42.7 & GB & $80-85$ & & & $\mathrm{OO}$ & o & & M & 0 & & M & & & & $\mathrm{T}$ & $\mathrm{T}$ & & & & $\mathrm{T}$ ? & & & $\begin{array}{l}\mathrm{dlg} ; \bmod \mathrm{fr} ; \mathrm{msz} / \mathrm{sb} ; \mathrm{kb} \\
\text { (cpx) }\end{array}$ \\
\hline $5 \mathrm{R}-1,56-60$ & 11 & 49.46 & GB & $85-95$ & & & $\mathrm{OO}$ & $\mathrm{T}-\mathrm{O}$ & & $\mathrm{OO}$ & $\mathrm{T}$ & & M & & & & & $\mathrm{x}$ & & & $\mathrm{T}$ & & boem & V/MV: pre; chl; zeo & $\begin{array}{l}\text { dlg; shrd/sb; mod fol; } \\
\text { kb (cpx; plg); fr }\end{array}$ \\
\hline $5 R-2,30-34$ & 7 & 50.7 & DU & $90-100$ & o & & & & $\mathrm{T}$ & & $\mathrm{T}$ & & $\mathrm{T}$ & $\mathrm{xx}$ & & $\mathrm{T}$ & o & & & $\mathrm{OO}$ & & & & $\begin{array}{l}\text { V: serp }+\mathrm{mt}+\mathrm{chl} / \\
\text { MV: serp }+\mathrm{mt}\end{array}$ & vnd mesh txt \\
\hline $6 \mathrm{R}-1,15-17$ & $4 \mathrm{~A}$ & 58.75 & GB & $85-90$ & & & $\mathrm{~T}-\mathrm{O}$ & $\mathrm{OO}$ & & M & $\mathrm{T}$ & & M & & & & $\mathrm{T}$ & $\mathrm{OO}$ & & $\mathrm{T}$ & o & & $\mathrm{T}$ & V/MV: pre; chl; zeo & $\begin{array}{l}\text { dlg; fr; incp sh/sb; kb } \\
\text { (cpx; plg) }\end{array}$ \\
\hline $6 \mathrm{R}-1,59-61$ & 11 & $\begin{array}{l}59.19 \\
5938\end{array}$ & DU & 100 & T & & & & $T$ & & & & $\mathrm{~T}$ & $\mathrm{xx}$ & & $\mathrm{T}$ & O & & & $\mathrm{T}$ & & $\mathrm{OO}$ & & $\mathrm{V}: \mathrm{serp}+\mathrm{mt} \pm \mathrm{br}$; arg & nnd mesh txt \\
\hline $\begin{array}{l}6 \mathrm{R}-1,78-80 \\
6 \mathrm{R}-2,50-54\end{array}$ & $\begin{array}{l}14 \\
5 \mathrm{~B}\end{array}$ & $\begin{array}{l}59.38 \\
60.6\end{array}$ & $\begin{array}{l}\mathrm{MG} \\
\mathrm{DU}\end{array}$ & $\begin{array}{c}100 \\
95-100\end{array}$ & 0 & & $\mathrm{~T}$ & & ${ }_{\mathrm{T}}^{\mathrm{T}}$ & $x$ & 0 & & $\begin{array}{l}X \\
T\end{array}$ & $\mathrm{xX}$ & & $\mathrm{T}$ & $\begin{array}{l}\mathrm{T} \\
\mathrm{O}\end{array}$ & & & 0 & & & & $\begin{array}{l}\text { V: chl + dio (def) } \\
\text { V: serp + mt + chl } \pm \text { cly }\end{array}$ & $\begin{array}{l}\text { shrd; fol } \\
\text { vnd mesh txt }\end{array}$ \\
\hline $6 R-3,26-31$ & IC & 61.57 & DU & $95-100$ & 0 & & & & $\mathrm{~T}$ & $\mathrm{~T}$ & & & 0 & $x x$ & & $\mathrm{~T}$ & $\mathrm{O}$ & & & 0 & & & & $\begin{array}{l}\text { /MV: serp + mt } \\
\text { V: serp }+\mathrm{mt}+\mathrm{chl} / \\
\text { MV: serp }+\mathrm{mt} \pm \mathrm{br}\end{array}$ & vnd mesh $\mathrm{txt}$ \\
\hline $6 \mathrm{R}-4,53-57$ & $2 \mathrm{~B}$ & 62.95 & DU & & OO & & & & $\mathrm{T}$ & & & & 0 & $x \mathrm{x}$ & & $\mathrm{o}$ & 0 & & & 0 & & & & $\begin{array}{l}\mathrm{V}: \operatorname{serp}+\mathrm{mt}+\mathrm{chl} / \mathrm{MV}: \\
\text { serp }+\mathrm{mt}+\mathrm{br}\end{array}$ & vnd mesh $\mathrm{xt}$ \\
\hline $\begin{array}{l}6 \mathrm{R}-4,116-120 \\
7 \mathrm{R}-1,59-63\end{array}$ & $\begin{array}{l}7 \\
7 \mathrm{~B}\end{array}$ & $\begin{array}{l}63.58 \\
68.79\end{array}$ & $\begin{array}{l}\mathrm{HZ} \\
\mathrm{IDU}\end{array}$ & $\begin{array}{l}95-100 \\
\text { Up to } 100\end{array}$ & $\mathrm{~T}$ & & & $\mathrm{~T}$ & 0 & & $\mathrm{~T}$ & $\mathrm{~T}$ & $\begin{array}{l}\mathrm{T} \\
\mathrm{O}\end{array}$ & $\begin{array}{l}X X \\
X X\end{array}$ & & $\stackrel{T}{T}$ & $\begin{array}{c}\mathrm{O} \\
\mathrm{OO}\end{array}$ & & $\mathrm{T}$ & $\begin{array}{c}\mathrm{T} \\
\mathrm{OO}\end{array}$ & & $\mathrm{T}$ & & $\begin{array}{l}\text { V/MV: serp + mt + chl } \\
\text { V: serp +mt } / M V: \text { serp } \\
\quad+m t+c h l \pm b r\end{array}$ & $\begin{array}{l}\text { porph vnd } \\
\text { vnd mesh txt }\end{array}$ \\
\hline $7 \mathrm{R}-2,50-55$ & 6 & 70.2 & DU & Up to 100 & $\mathrm{~T}$ & $\mathrm{~T}$ ? & $\mathrm{T}$ & $\mathrm{T}$ ? & $\mathrm{T}$ & $\mathrm{o}$ & $\mathrm{T}$ & & $\mathrm{OO}$ & $\mathrm{XX}$ & & $\mathrm{T}$ & $\mathrm{OO}$ & & & $\mathrm{T}$ & & & & $\begin{array}{l}\text { V: serp }+\mathrm{mt} \pm \mathrm{tr} \pm \mathrm{chl} / \\
\text { MV: } \operatorname{serp}+\mathrm{mt} \pm \mathrm{br}\end{array}$ & vnd mesh txt \\
\hline $7 \mathrm{R}-3,2-8$ & IB & 71.22 & DU? & 100 & & & & & $\mathrm{~T}$ & $\mathrm{~T}$ & & & $\mathrm{~T}$ & $\mathrm{xx}$ & & $\mathrm{o}$ & $\mathrm{O}$ & & & $\mathrm{o}$ & & $\mathrm{OO}$ & & $\begin{array}{l}\text { V/MV: serp }+m t \pm b r / \\
\text { V: arg }\end{array}$ & vnd mesh txt \\
\hline $8 R-3,7-10$ & 1 & 80.87 & DU & Up to 100 & $\mathrm{~T}$ & & & & $\mathrm{~T}$ & & & & $\mathrm{~T}$ & $\mathrm{xx}$ & & $\mathrm{T}$ & 0 & & & $\mathrm{OO}$ & & & & $\begin{array}{l}\mathrm{V}: \text { serp }+\mathrm{mt}+\mathrm{chl} / \\
\mathrm{MV}: \operatorname{serp}+\mathrm{mt}\end{array}$ & vnd mesh txt \\
\hline $8 R-4,59-60$ & 9 & 82.89 & MG & 100 & & & $\mathrm{~T}$ & & $\mathrm{~T}$ & M & 0 & & $\mathrm{x}$ & $\mathrm{OO}$ & & & $\mathrm{T}$ & & & $\mathrm{O}$ & & & 0 & $\begin{array}{l}\text { V/MV: chl + serp/atg? } \\
\text { (def); cly + Fe-hox }\end{array}$ & $\begin{array}{l}\text { shrd; fol; } \mathrm{kb} \text { (cpx; chl; } \\
\text { tre); cont spt }\end{array}$ \\
\hline $8 R-4,135-141$ & 19 & 83.65 & DU & $95-100$ & $\mathrm{~T}$ & & & & $\mathrm{~T}$ & $\mathrm{~T}$ & $\mathrm{~T}$ & & $\mathrm{~T}$ & $\mathrm{xx}$ & & $\mathrm{T}$ & $\mathrm{o}$ & & & $\mathrm{T}$ & & 0 & & $\begin{array}{l}\mathrm{V}: \arg +\mathrm{tr}+\mathrm{chl} / \mathrm{MV}: \\
\text { serp + mt }\end{array}$ & mesh txt \\
\hline
\end{tabular}

Notes: Mineral abbreviations: olv = olivine; opx =orthopyroxene; $\mathrm{cpx}=$ clinopyroxene; $\mathrm{plg}=$ plagioclase; sp/ox = spinel/oxide; $\mathrm{tr}=$ tremolitic amphibole; scpx = secondary clinopyroxene; spl = secondary plagioclase; chl = chlorite; serp $=$ serpentine minerals; $\mathrm{tlc}=\mathrm{talc} ; \mathrm{br}=$ brucite; $\mathrm{mt} / \mathrm{ox}=$ secondary magnetite or oxides; pre $=$ prehnite; $\mathrm{hgr}=$ hydrogrossular $($ including transitional phases to prehnite); cly $=$ clay minerals; zeo $=$ zeolite; $\mathrm{cz} / \mathrm{ep}=$ clinozoisite/epidote; $\mathrm{ccl} /$ arg = calcite/ aragonite; hox = hydroxides; boem = boehmite. Lithologic abbreviations: $\mathrm{HZ}=$ harzburgite; $\mathrm{DU}=$ dunite; $\mathrm{IDU}=$ impregnated dunite; $\mathrm{TRO}=$ troctolite; $\mathrm{OLGB}=$ olivine gabbro; $\mathrm{GB}=$ gabbro; $\mathrm{MGB}=$ metagabbro. Modal percentages: $\mathrm{T}=\langle 1 \% ; \mathrm{O}=$ to $5 \% ; \mathrm{OO}=5-15 \% ; \mathrm{M}=15-30 \% ; \mathrm{X}=30-50 \% ; \mathrm{XX}=>50 \%$. Vein assemblages are divided into discrete vein $(\mathrm{V})$ phases and microvein (MV) phases; $\mathrm{MG} / \mathrm{MV}=$ multiple generations of microveins are present. Peridotite textures: porph mesh txt $=$ porphyroclastic mesh textured, which can be veined (vnd) or strongly veined (svnd), and vnd mesh txt $=$ veined mesh textured. Textures of mafic rocks: $\mathrm{wk}$ ir $=$ weakly fractured; mod fr $=$ moderately fractured; $\mathrm{fr}=\mathrm{highl}$ fractured; shrd $=$ sheared; $\mathrm{sb}=$ shear bands; incip $\mathrm{sh}=$ incipient shearing; incip sh/sb $=$ incipient shearing with local development of shear bands; msz/sb $=$ microshear zones and shear bands; def veins
$=$ veins are deformed; $\mathrm{kb}=$ kink bands in mineral indicated in parentheses; $\mathrm{wk}$ fol $=$ weakly foliated; mod fol = moderately foliated; fol $=$ strongly foliated; dial $=$ coarse-grained to pegmatitic diallage-textured gabbro; cont spt = contact = veins are deformed; $\mathrm{kb}=\mathrm{kin} \mathrm{k}$ bands in mine
to serpentinized peridotite observed in sample. 


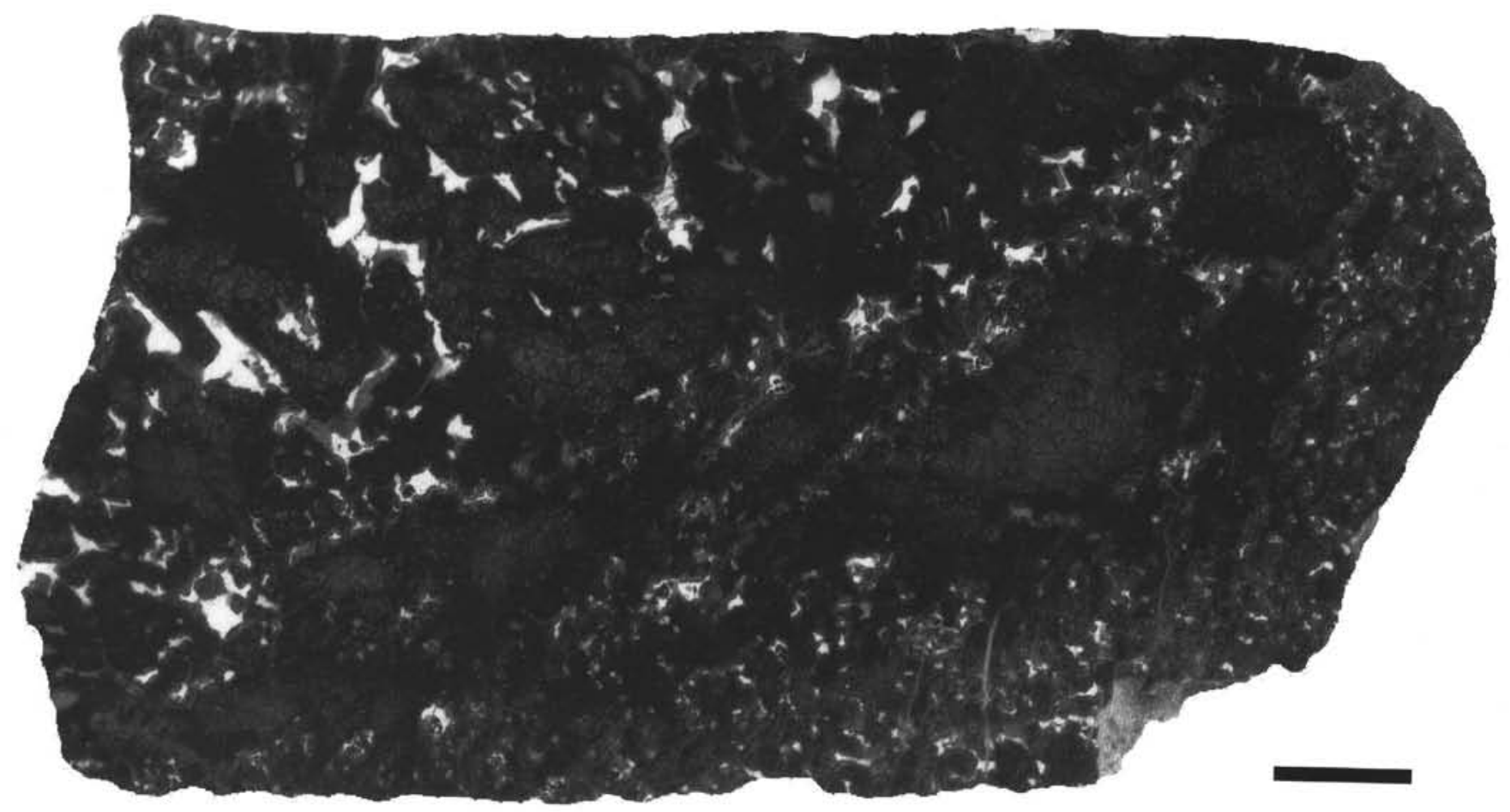

Figure 2. Plagioclase and clinopyroxene impregnations in Site 895 dunite Sample 147-895D-7R-1 (Piece 8, 44-56 cm). Scale bar is $1 \mathrm{~cm}$.

ture-fillings in originally larger olivine grains and along grain boundaries. In more extensively altered samples, the original olivine grains are marked by subrounded to rounded, completely pseudomorphed patches, consisting of mesh-textured lizardite \pm chrysotile, finegrained magnetite, brucite, and clays. When abundant, chlorite is generally concentrated in fibrous patches, often associated with veins. It is usually colorless to very pale yellowish green, with normal to anomalous blue birefringence.

Serpentine generally makes up more than 50 modal\% of the sampled dunites and harzburgites. The first stage of olivine replacement takes place along grain boundaries and fractures of originally larger olivine crystals. As the process continues, a well-developed mesh texture forms, and the olivine grain size decreases until no more olivine remains. From the uniform extinction of clusters of several olivine kernels, the original grain size of olivine can be estimated to be $1-4 \mathrm{~mm}$. Secondary very fine-grained magnetite is either disseminated or concentrated in trails along the anastomosing serpentine network. Yellowish to red-brownish patches of clay minerals locally fill the cores of the pseudomorphed olivine grains.

The results of XRD analyses of serpentine separates from representative rock- and vein-serpentine show similar mineralogies and indicate a lizardite- and chrysotile-dominated mixture, with a prevalence of lizardite in the rock-serpentine separates (Wicks and O'Hanley, 1988; Whittaker and Zussman, 1956). High-intensity reflections were observed at $d(\AA)=7.30-7.35 ; 4.60-4.61 ; 3.65-3.66 ; 2.50$ $2.51 ; 2.45-2.46$ (not always detected), $1.53-1.54$; and $1.50-1.51$ (not always detected). Reflections with relatively lower intensities were rarely observed at $\mathrm{d}(\AA)=2.52-2.53 ; 2.16-2.17$; and $1.56-1.57$. The 330 reflections at $1.56-1.57 \AA$ are particularly unique to antigorite (Whittaker and Zussman, 1956), and may indicate the presence of varying amounts of this phase. Preliminary investigations using highresolution transmission electron microscopy (HRTEM) confirm the presence of antigorite in these samples (Früh-Green et al., 1995). The antigorites show relatively uniform, short wave-length HRTEM images, indicative of crystallization temperatures above approximately $400^{\circ} \mathrm{C}$ (Mellini et al., 1987).

\section{Orthopyroxene}

Enstatite generally occurs as porphyroclasts, and is characterized by a large variation in grain size, ranging from less than $1 \mathrm{~mm}$ to more than $20 \mathrm{~mm}$, with an average grain size of approximately $5 \mathrm{~mm}$. A spectrum of morphologies is observed, varying from subrounded, nearly isometric grains, to tabular crystals, to irregularly shaped, densely fractured and veined patches altered to lizardite and/or chrysotile, tremolite, chlorite, and locally talc. A preferred orientation of the orthopyroxene porphyroclasts is poorly defined on a thin section scale. Inclusions (predominantly olivine and clinopyroxene) occur locally in relatively fresh orthopyroxene grains. Enstatite and its alteration products commonly make up 10-15 modal\% of the harzburgites and constitute trace components in the dunitic rocks. Grain boundaries to the surrounding olivine grains are often ill-defined and are interconnected by a series of microfractures filled with antigorite(?) and chlorite.

Orthopyroxene alteration is highly variable and ranges from rare relicts of nearly fresh, individual grains in some harzburgites to completely altered pseudomorphs in harzburgites and dunites. Alteration is selective and produced several alteration assemblages, and is generally higher in dunites and invariably more pervasive in veined samples. Replacement is characterized by thin talc coronas and fracturefillings (generally observed in nearly fresh grains; PI. 1, Fig. 1), by fine-grained tremolite and tremolite-chlorite overgrowths, and by undeformed or slightly kinked lizardite and/or chrysotile forming bastite pseudomorphs (PI. 1, Fig. 2). In the bastite pseudomorphs, elongated grains of clinopyroxene and tremolite commonly have preferred orientation along the (100) crystallographic plane of the original enstatite grains.

\section{Clinopyroxene}

Primary augite contents in the ultramafic rocks rarely exceed 1 modal \%. Primary clinopyroxene occurs in multiple forms: as pseudovermicular intergrowths with spinel; as coarse-grained, locally isolated, undeformed poikilitic crystals in impregnated dunites (Pl. 1., Fig. 3 ); as fine-grained coronas (up to a few $\mu \mathrm{m}$ ) outlining altered plagio- 
clase in impregnated dunites (Pl. 1., Fig. 3); and as fine-grained (up to $1 \mathrm{~mm}$ ) interstitial crystals along the margins of enstatite grains. Alteration is moderate and consists of very fine-grained magnetite microfracture-fillings and fine-grained, colorless, fibrous tremolite overgrowths. Coarse-grained, undeformed poikilitic primary clinopyroxene often contains diffuse, very fine-grained magnetite exsolutions. Tremolite can extend from clinopyroxene margins to the neighboring grains, forming wide pervasive patches, locally associated with abundant chlorite. Fibrous tremolite enrichments are also observed along some late aragonite crosscutting veins and occur, together with chlorite, as patches ( $1-2 \mathrm{~mm}$ across) between the serpentine-olivine groundmass and the vein. Secondary diopside is less common, occurring locally as acicular grains in serpentine and in serpentine-chlorite crack-seal veins (see Site 895 fig. 20 in Gillis, Mével, Allan, et al., 1993).

\section{Spinel}

Disseminated spinels in the harzburgites and dunites are deep, dark red- to brownish red-colored $\mathrm{Mg}$-Al-chromites, typically characterized by alteration rims and irregular replacements of submicroscopic intergrowths of opaque ferrite-chromite with chlorite, and/or fine-grained magnetite overgrowths. In both harzburgites and dunites, spinel exhibits a wide spectrum of shapes, ranging from rare, euhedral isometric grains, to subrounded, predominantly fine-grained granular crystals, to irregular elongate, or atoll-like forms. Extremely irregularly shaped grain boundaries and pseudo-vermicular intergrowths with primary augite are common. Spinel abundancies only locally exceed 1 modal\%. Slightly enriched zones occur in dunites and locally exhibit elongated spinel grains with preferred orientation. Alteration phases include ferrite-chromite, magnetite (locally forming very fine-grained radiating rims interfingered with the serpentine groundmass), localized overgrowths of fibrous chlorite patches, and fracture-fillings of brucite, serpentine and chlorite.

\section{Plagioclase}

Plagioclase impregnations are common in the dunitic rocks recovered at Site 895 and range from trace amounts of interstitial plagioclase to a few modal\%. They typically have a turbid appearance and are partially to completely replaced by secondary phases. They are generally undeformed, with only local, very weak undulatory extinction, and rarely show well-defined twinning planes. Replacement textures are characterized by a thin $(10-50 \mu \mathrm{m})$ primary clinopyroxene corona, outlining a chlorite \pm serpentine \pm nearly opaque prehnite/hydrogrossular pseudomorph assemblage after plagioclase ( $\mathrm{Pl}$. 1, Fig. 3). Occasionally the replacement is monomineralic and is composed of blue birefringent chlorite rimmed by radiating fibers of gray birefringent chlorite.

\section{Mineral Chemistry of Primary and Secondary Phases}

The chemical composition of relict olivine is relatively homogeneous throughout the samples, with no detectable core-rim variations. Electron microprobe analyses indicate constant high $\mathrm{Mg}$ contents with approximately $90 \mathrm{~mol} \%$ forsterite (Fo) and high Ni contents, ranging from $0.35-0.44 \mathrm{wt} \% \mathrm{NiO}$, typical of mantle peridotites and dunite cumulates (Bonatti and Michael, 1989; Bonatti et al., 1990; see Table 2). Ca contents range from 0.02 to $0.12 \mathrm{wt} \% \mathrm{CaO}$. In one plagioclase-impregnated dunite sample (147-895C-4R-1 (Piece $2 \mathrm{~A}, 13-16 \mathrm{~cm})$ ) olivine shows slightly lower forsterite $\left(\mathrm{Fo}_{88}\right)$ and $\mathrm{Ni}$ contents $(0.32-0.37 \mathrm{wt} \% \mathrm{NiO})$. Individual plagioclase grains have constant compositions, with an average An content of 85 mole percent.

Primary orthopyroxene compositions range from 79 to $83 \mathrm{~mol} \%$ enstatite (En), 3 to $7 \mathrm{~mol} \%$ wollastonite (Wo) and 6 to $9 \mathrm{~mol} \%$ Ferrosilite (Fs), with a moderate $\mathrm{Al}_{2} \mathrm{O}_{3}$ content of 2.10 to $2.51 \mathrm{wt} \%$ (Table 3 ). Primary and secondary clinopyroxenes show two distinct
Table 2. Representative olivine compositions in Site $\mathbf{8 9 5}$ ultramafic and mafic lithologies.

\begin{tabular}{|c|c|c|c|c|c|c|c|c|}
\hline $\begin{array}{l}\text { Hole: } \\
\text { Core, section: } \\
\text { Interval (cm): } \\
\text { Piece: } \\
\text { Lithology: }\end{array}$ & $\begin{array}{c}895 \mathrm{C} \\
4 \mathrm{R}-3 \\
13-16 \\
2 \mathrm{~A} \\
\text { IDU }\end{array}$ & $\begin{array}{c}895 \mathrm{D} \\
2 \mathrm{R}-2 \\
29-32 \\
2 \\
\mathrm{HZ}\end{array}$ & $\begin{array}{c}895 \mathrm{D} \\
2 \mathrm{R}-2 \\
29-32 \\
2 \\
\mathrm{HZ}\end{array}$ & $\begin{array}{c}895 \mathrm{D} \\
7 \mathrm{R}-1 \\
81-84 \\
13 \\
\text { TRO }\end{array}$ & $\begin{array}{c}895 \mathrm{D} \\
7 \mathrm{R}-1 \\
81-84 \\
13 \\
\text { TRO }\end{array}$ & $\begin{array}{c}895 \mathrm{E} \\
1 \mathrm{R}-1 \\
83-87 \\
9 \\
\text { OLGB }\end{array}$ & $\begin{array}{c}895 \mathrm{D} \\
4 \mathrm{R}-2 \\
126-129 \\
18 \\
\mathrm{HZ}\end{array}$ & $\begin{array}{c}895 \mathrm{D} \\
4 \mathrm{R}-2 \\
126-129 \\
18 \\
\mathrm{HZ}\end{array}$ \\
\hline $\begin{array}{l}\mathrm{SiO}_{2} \\
\mathrm{TiO}_{2} \\
\mathrm{Al}_{2} \mathrm{O}_{3} \\
\mathrm{Cr}_{2} \mathrm{O}_{3} \\
\mathrm{FeO} \\
\mathrm{MnO} \\
\mathrm{NiO} \\
\mathrm{MgO} \\
\mathrm{CaO} \\
\mathrm{Na}_{2} \mathrm{O} \\
\mathrm{K}_{2} \mathrm{O} \\
\mathrm{Cl} \\
\text { Total }\end{array}$ & $\begin{array}{r}40.92 \\
0.00 \\
0.02 \\
0.00 \\
11.15 \\
0.22 \\
0.37 \\
48.52 \\
0.03 \\
0.02 \\
0.00 \\
0.03 \\
101.28\end{array}$ & $\begin{array}{r}40.71 \\
0.01 \\
0.02 \\
0.01 \\
8.89 \\
0.09 \\
0.40 \\
49.19 \\
0.02 \\
0.00 \\
0.01 \\
0.02 \\
99.37\end{array}$ & $\begin{array}{r}40.66 \\
0.00 \\
0.03 \\
0.04 \\
8.86 \\
0.14 \\
0.44 \\
49.16 \\
0.07 \\
0.02 \\
0.01 \\
0.02 \\
99.43\end{array}$ & $\begin{array}{r}40.84 \\
0.01 \\
0.03 \\
0.01 \\
10.53 \\
0.19 \\
0.35 \\
48.65 \\
0.08 \\
0.00 \\
0.00 \\
0.01 \\
100.70\end{array}$ & $\begin{array}{r}40.79 \\
0.00 \\
0.00 \\
0.00 \\
10.61 \\
0.21 \\
0.39 \\
48.70 \\
0.10 \\
0.03 \\
0.02 \\
0.00 \\
100.86\end{array}$ & $\begin{array}{r}40.98 \\
0.01 \\
0.04 \\
0.03 \\
11.16 \\
0.14 \\
0.29 \\
48.43 \\
0.05 \\
0.01 \\
0.01 \\
0.01 \\
101.16\end{array}$ & $\begin{array}{r}41.14 \\
0.01 \\
0.02 \\
0.03 \\
9.29 \\
0.18 \\
0.39 \\
49.96 \\
0.10 \\
0.00 \\
0.00 \\
0.00 \\
101.12\end{array}$ & $\begin{array}{r}40.93 \\
0.01 \\
0.04 \\
0.01 \\
9.16 \\
0.13 \\
0.43 \\
49.90 \\
0.10 \\
0.01 \\
0.00 \\
0.00 \\
100.71\end{array}$ \\
\hline $\begin{array}{l}\mathrm{Si} \\
\mathrm{Ti} \\
\mathrm{Al} \\
\mathrm{Cr} \\
\mathrm{Fe}^{2+} \\
\mathrm{Mn} \\
\mathrm{Ni} \\
\mathrm{Mg} \\
\mathrm{Ca} \\
\mathrm{Na} \\
\mathrm{K}\end{array}$ & $\begin{array}{l}1.00 \\
0.00 \\
0.00 \\
0.00 \\
0.23 \\
0.00 \\
0.01 \\
1.76 \\
0.00 \\
0.00 \\
0.00\end{array}$ & $\begin{array}{l}1.00 \\
0.00 \\
0.00 \\
0.00 \\
0.18 \\
0.00 \\
0.01 \\
1.80 \\
0.00 \\
0.00 \\
0.00\end{array}$ & $\begin{array}{l}1.00 \\
0.00 \\
0.00 \\
0.00 \\
0.18 \\
0.00 \\
0.01 \\
1.80 \\
0.00 \\
0.00 \\
0.00\end{array}$ & $\begin{array}{l}1.00 \\
0.00 \\
0.00 \\
0.00 \\
0.22 \\
0.00 \\
0.01 \\
1.77 \\
0.00 \\
0.00 \\
0.00\end{array}$ & $\begin{array}{l}1.00 \\
0.00 \\
0.00 \\
0.00 \\
0.22 \\
0.00 \\
0.01 \\
1.77 \\
0.00 \\
0.00 \\
0.00\end{array}$ & $\begin{array}{l}1.00 \\
0.00 \\
0.00 \\
0.00 \\
0.23 \\
0.00 \\
0.01 \\
1.76 \\
0.00 \\
0.00 \\
0.00\end{array}$ & $\begin{array}{l}1.00 \\
0.00 \\
0.00 \\
0.00 \\
0.19 \\
0.00 \\
0.01 \\
1.80 \\
0.00 \\
0.00 \\
0.00\end{array}$ & $\begin{array}{l}0.99 \\
0.00 \\
0.00 \\
0.00 \\
0.19 \\
0.00 \\
0.01 \\
1.81 \\
0.00 \\
0.00 \\
0.00\end{array}$ \\
\hline $\begin{array}{l}\text { Forsterite } \\
\text { Fayalite }\end{array}$ & $\begin{array}{l}0.88 \\
0.11\end{array}$ & $\begin{array}{l}0.90 \\
0.09\end{array}$ & $\begin{array}{l}0.90 \\
0.09\end{array}$ & $\begin{array}{l}0.89 \\
0.11\end{array}$ & $\begin{array}{l}0.89 \\
0.11\end{array}$ & $\begin{array}{l}0.88 \\
0.11\end{array}$ & $\begin{array}{l}0.90 \\
0.09\end{array}$ & $\begin{array}{l}0.90 \\
0.09\end{array}$ \\
\hline
\end{tabular}

Note: Mineral abbreviations as in Table 1.

ranges in chemical composition. Primary clinopyroxenes are distinguished by a lower average $\mathrm{CaO}$ content, and clearly higher $\mathrm{Al}, \mathrm{Cr}$, and $\mathrm{Na}$ contents. They are augites and have an average $\mathrm{CaO}$ content of $23 \mathrm{wt} \%$ (average Wo 41), with 2.68-3.42 wt\% $\mathrm{Al}_{2} \mathrm{O}_{3}, 1.01-1.69$ wt $\% \mathrm{Cr}_{2} \mathrm{O}_{3}$, and $0.03-0.49 \mathrm{wt} \% \mathrm{Na}_{2} \mathrm{O}$. Secondary diopsides are characterized by an average $\mathrm{CaO}$ content of $26 \mathrm{wt} \%$ (average Wo 49), and $0.03-0.91$ wt $\% \mathrm{Al}_{2} \mathrm{O}_{3},<0.01-0.15$ wt $\% \mathrm{Cr}_{2} \mathrm{O}_{3}$, and $0.02-0.08$ wt $\%$ $\mathrm{Na}_{2} \mathrm{O}$ (see Table 3 ).

The composition of primary spinel in the Site 895 ultramafic rocks is very similar to that observed in previously dredged samples at Hess Deep (Hekinian et al., 1993). It is characterized by low $\mathrm{X}_{\mathrm{Fe}^{3+}}$ $\left[=\mathrm{Fe}^{3+} /\left(\mathrm{Al}+\mathrm{Cr}+\mathrm{Fe}^{3+}\right)=0.02-0.03\right]$, high $\mathrm{X}_{\mathrm{Cr}}\left[=\mathrm{Cr} /\left(\mathrm{Al}+\mathrm{Cr}+\mathrm{Fe}^{3+}\right)\right.$ $=0.51-0.53]$ and constant, very low $\mathrm{TiO}_{2}\left(0.03-0.05 \mathrm{wt} \% \mathrm{TiO}_{2}\right)$ in the harzburgites. Higher $\mathrm{TiO}_{2}-$ and $\mathrm{X}_{\mathrm{Fe}^{3+}}$ contents $(0.44-0.48 \mathrm{wt} \%$ $\mathrm{TiO}_{2}, \mathrm{X}_{\mathrm{Fe}^{3+}}=0.05-0.07$ ) have been observed in Site 895 dunitic rocks (Allan and Leg 147 Scientific Party, 1993) and may reflect partial exchange due to melt contamination (Allan and Leg 147 Scientific Party, 1993; Dick and Bullen, 1984). Optically undetectable opaque replacements, composed of submicroscopic intergrowths of ferrite-chromite and possibly chlorite have been detected by electron backscatter analysis, with non-stoichiometric compositions and variable $\mathrm{SiO}_{2}$ and $\mathrm{H}_{2} \mathrm{O}$ contents (see Table 4).

Serpentine-group minerals characteristically have variable $\mathrm{Fe}_{\mathrm{tos}}$ (total $\mathrm{Fe}=\mathrm{Fe}_{2} \mathrm{O}_{3}+\mathrm{FeO}$ ) contents (Wicks and O'Hanley, 1988). Although iron is preferentially incorporated in magnetite during serpentinization of olivine and pyroxene, lizardite compositions with up to or more than $16 \mathrm{wt} \%$ total iron, calculated as FeO, have been measured in natural samples (Wicks and Plant, 1979; D. O'Hanley, pers. comm., 1994). In contrast to lizardite, the chrysotile structure generally accommodates less iron (Wicks and O'Hanley, 1988), although some chrysotile samples with up to $8 \mathrm{wt} \%$ total iron have been reported by Wicks and Plant (1979). The measured serpentine compositions in the Site 895 harzburgites and dunites indicate a high $\mathrm{Fe}_{\text {tot }}(\mathrm{Ta}$ ble 5 ). The serpentine-bastite pseudomorphs exhibit relatively constant total iron contents of 3-5 wt\%, whereas iron contents in mesh serpentines after olivine are typically more variable (4-8 $\left.\mathrm{wt} \% \mathrm{Fe}_{\text {tot }}\right)$. 
Table 3. Representative primary and secondary pyroxene compositions in Site 895 peridotites.

\begin{tabular}{|c|c|c|c|c|c|c|c|c|c|c|c|c|c|c|}
\hline \multirow{7}{*}{$\begin{array}{l}\text { Hole: } \\
\text { Core, section: } \\
\text { Interval }(\mathrm{cm}) \\
\text { Piece: } \\
\text { Lithology: } \\
\text { Association: }\end{array}$} & \multicolumn{7}{|c|}{ Primary clinopyroxene } & \multicolumn{3}{|c|}{ Secondary clinopyroxene } & \multicolumn{4}{|c|}{ Orthopyroxene } \\
\hline & $895 \mathrm{C}$ & $895 \mathrm{C}$ & $895 \mathrm{C}$ & $895 \mathrm{C}$ & 895D & 895D & 895D & $895 \mathrm{C}$ & $895 \mathrm{C}$ & 895D & 895D & 895D & 895D & 895D \\
\hline & $4 \mathrm{R}-3$ & $4 \mathrm{R}-3$ & $4 \mathrm{R}-3$ & $4 \mathrm{R}-3$ & $2 \mathrm{R}-2$ & $4 \mathrm{R}-2$ & $4 \mathrm{R}-2$ & $4 \mathrm{R}-2$ & $4 \mathrm{R}-3$ & $4 \mathrm{R}-2$ & $2 \mathrm{R}-2$ & $2 \mathrm{R}-2$ & $2 \mathrm{R}-2$ & $4 \mathrm{R}-2$ \\
\hline & $13-16$ & $13-16$ & $13-16$ & $13-16$ & $29-32$ & $126-129$ & $126-129$ & $52-54$ & $13-16$ & $126-129$ & $29-32$ & $29-32$ & $29-32$ & $126-129$ \\
\hline & $2 \mathrm{~A}$ & $2 \mathrm{~A}$ & $2 \mathrm{~A}$ & $2 \mathrm{~A}$ & 2 & 18 & 18 & ID & $2 \mathrm{~A}$ & 18 & 2 & 2 & 2 & 18 \\
\hline & IDU & IDU & IDU & IDU & $\mathrm{HZ}$ & $\mathrm{HZ}$ & $\mathrm{HZ}$ & IDU & IDU & $\mathrm{HZ}$ & $\mathrm{HZ}$ & $\mathrm{HZ}$ & $\mathrm{HZ}$ & $\mathrm{HZ}$ \\
\hline & rim to plg & rim to plg & poik. & rim to plg & with opx & with sp & interstitial & vein & vein & vein & & & & \\
\hline $\mathrm{SiO}_{2}$ & 52.01 & 51.36 & 51.47 & 50.64 & 52.04 & 52.56 & 49.66 & 54.98 & 53,04 & 53.81 & 55.38 & 55.73 & 55.84 & 56.27 \\
\hline $\mathrm{TiO}_{2}$ & 1.00 & 0.88 & 0.61 & 0.94 & 0.03 & 0.03 & 0.02 & 0.00 & 0.02 & 0.00 & 0.02 & 0.02 & 0.02 & 0.02 \\
\hline $\mathrm{Al}_{2}^{2} \mathrm{O}_{3}$ & 2.95 & 3.09 & 3.34 & 3.36 & 2.68 & 2.94 & 3.03 & 0.03 & 0.23 & 0.91 & 2.25 & 2.47 & 2.20 & 2.14 \\
\hline $\mathrm{Cr}_{2} \mathrm{O}_{3}$ & 1.01 & 1.07 & 1.11 & 1.18 & 1.07 & 1.48 & 1.31 & 0.00 & 0.01 & 0.15 & 0.81 & 0.83 & 0.71 & 0.72 \\
\hline $\mathrm{Fe}_{2} \mathrm{O}_{3}$ & 0.00 & 0.00 & 0.00 & 0.00 & 0.00 & 0.00 & 0.00 & 0.22 & 0.00 & 0.00 & 0.17 & 0.00 & 0.64 & 1.18 \\
\hline $\mathrm{FeO}$ & 2.92 & 3.17 & 3.42 & 3.01 & 2.71 & 2.81 & 4.04 & 1.49 & 2.62 & 3.30 & 5.19 & 5.81 & 5.25 & 4.83 \\
\hline $\mathrm{MnO}$ & 0.12 & 0.18 & 0.14 & 0.14 & 0.08 & 0.10 & 0.09 & 0.11 & 0.10 & 0.21 & 0.10 & 0.13 & 0.14 & 0.11 \\
\hline $\mathrm{NiO}$ & 0.05 & 0.05 & 0.05 & 0.03 & 0.10 & 0.07 & 0.04 & 0.00 & 0.00 & 0.04 & 0.08 & 0.13 & 0.11 & 0.07 \\
\hline $\mathrm{MgO}$ & 16.56 & $\begin{array}{l}0.05 \\
16.49\end{array}$ & 18.12 & $\begin{array}{l}0.03 \\
15.83\end{array}$ & $\begin{array}{l}0.10 \\
18.03\end{array}$ & 17.98 & $\begin{array}{l}0.04 \\
18.61\end{array}$ & $\begin{array}{l}0.00 \\
17.67\end{array}$ & $\begin{array}{l}0.00 \\
18.23\end{array}$ & $\begin{array}{r}16.04 \\
16.57\end{array}$ & $\begin{array}{r}31.42 \\
31.42\end{array}$ & $\begin{array}{l}0.13 \\
32.48\end{array}$ & 33.16 & $\begin{array}{r}0.07 \\
33.64\end{array}$ \\
\hline $\mathrm{CaO}$ & $\begin{array}{l}10.50 \\
23.61\end{array}$ & 23.52 & 20.71 & 23.53 & 22.70 & 22.35 & $\begin{array}{l}21.92 \\
21.92\end{array}$ & 26.30 & 24.44 & 25.88 & $\begin{array}{r}3.42 \\
3.69\end{array}$ & $\begin{array}{l}1.76 \\
1.76\end{array}$ & 1.63 & $\begin{array}{l}3.04 \\
1.69\end{array}$ \\
\hline $\mathrm{Na}_{2} \mathrm{O}$ & $\begin{array}{r}25.01 \\
0.37\end{array}$ & 0.38 & $\begin{array}{r}0.11 \\
0.49\end{array}$ & 0.42 & $\begin{array}{r}2.70 \\
0.03\end{array}$ & 0.38 & 0.35 & $\begin{array}{r}20.00 \\
0.08\end{array}$ & 0.06 & $\begin{array}{r}2.80 \\
0.06\end{array}$ & 0.02 & $\begin{array}{l}1.70 \\
0.02\end{array}$ & 0.01 & $\begin{array}{l}1.09 \\
0.03\end{array}$ \\
\hline $\begin{array}{l}\mathrm{Na}_{2} \mathrm{O} \\
\mathrm{K}_{2} \mathrm{O}\end{array}$ & 0.00 & $\begin{array}{l}0.38 \\
0.01\end{array}$ & $\begin{array}{l}0.49 \\
0.04\end{array}$ & $\begin{array}{l}0.42 \\
0.02\end{array}$ & 0.03 & 0.01 & 0.01 & $\begin{array}{l}0.08 \\
0.03\end{array}$ & $\begin{array}{l}0.00 \\
0.04\end{array}$ & 0.01 & $\begin{array}{l}0.02 \\
0.01\end{array}$ & $\begin{array}{l}0.02 \\
0.00\end{array}$ & 0.02 & 0.01 \\
\hline $\mathrm{Cl}$ & 0.02 & 0.01 & 0.05 & 0.03 & 0.01 & 0.01 & 0.12 & 0.03 & 0.20 & 0.03 & 0.02 & 0.02 & 0.02 & 0.01 \\
\hline Total & 100.63 & 100.22 & 99.53 & 99.12 & 99.50 & 100.72 & 99.18 & 100.93 & $\begin{array}{l}98.20 \\
98.92\end{array}$ & 100.96 & $\begin{array}{r}9.02 \\
99.16\end{array}$ & $\begin{array}{r}9.02 \\
99.40\end{array}$ & 99.74 & 100.72 \\
\hline $\mathrm{Si}$ & 1.89 & 1.88 & 1.89 & 1.88 & 1.91 & 1.90 & 1.84 & 1.98 & 1.96 & 1.96 & 1.94 & 1.94 & 1.94 & 1.93 \\
\hline $\mathrm{Ti}$ & 0.03 & 0.02 & 0.02 & 0.03 & 0.00 & 0.00 & 0.00 & 0.00 & 0.00 & 0.00 & 0.00 & 0.00 & 0.00 & 0.00 \\
\hline $\mathrm{Al}$ & 0.13 & 0.13 & 0.14 & 0.15 & 0.11 & 0.12 & 0.13 & 0.00 & 0.01 & 0.04 & 0.09 & 0.10 & 0.09 & 0.09 \\
\hline $\mathrm{Cr}$ & 0.03 & 0.03 & 0.03 & 0.03 & 0.03 & 0.04 & 0.04 & 0.00 & 0.00 & 0.00 & 0.02 & 0.02 & 0.02 & 0.02 \\
\hline $\mathrm{Fe}^{3+}$ & 0.00 & 0.00 & 0.00 & 0.00 & 0.00 & 0.00 & 0.00 & 0.01 & 0.00 & 0.00 & 0.00 & $\begin{array}{l}0.02 \\
0.00\end{array}$ & 0.02 & 0.03 \\
\hline $\mathrm{Fe}^{2+}$ & $\begin{array}{l}0.00 \\
0.09\end{array}$ & 0.09 & 0.10 & $\begin{array}{l}0.00 \\
0.09\end{array}$ & 0.08 & 0.08 & $\begin{array}{l}0.00 \\
0.12\end{array}$ & $\begin{array}{l}0.01 \\
0.05\end{array}$ & $\begin{array}{l}0.00 \\
0.08\end{array}$ & $\begin{array}{l}0.00 \\
0.10\end{array}$ & $\begin{array}{l}0.15 \\
0.15\end{array}$ & $\begin{array}{l}0.00 \\
0.17\end{array}$ & $\begin{array}{l}0.02 \\
0.15\end{array}$ & $\begin{array}{l}0.14 \\
0.14\end{array}$ \\
\hline $\mathrm{Mn}$ & 0.00 & 0.01 & $\begin{array}{l}0.10 \\
0.00\end{array}$ & 0.00 & 0.00 & 0.00 & 0.00 & 0.00 & 0.00 & $\begin{array}{l}0.10 \\
0.01\end{array}$ & $\begin{array}{l}0.13 \\
0.00\end{array}$ & 0.00 & $\begin{array}{l}0.13 \\
0.00\end{array}$ & $\begin{array}{l}0.14 \\
0.00\end{array}$ \\
\hline $\mathrm{Ni}$ & 0.00 & 0.00 & 0.00 & 0.00 & 0.00 & 0.00 & 0.00 & 0.00 & 0.00 & 0.00 & 0.00 & 0.00 & 0.00 & 0.00 \\
\hline $\mathrm{Mg}$ & 0.89 & 0.90 & 0.99 & 0.87 & 0.98 & 0.97 & 1.03 & 0.95 & 1.01 & 0.90 & 1.64 & $\begin{array}{l}1.69 \\
\text { (n) }\end{array}$ & 1.71 & 1.72 \\
\hline $\mathrm{Ca}$ & 0.92 & 0.92 & 0.81 & 0.93 & 0.89 & 0.86 & 0.87 & 1.02 & 0.97 & 1.01 & 0.14 & 0.06 & 0.06 & 0.06 \\
\hline $\mathrm{Na}$ & 0.03 & 0.02 & 0.04 & 0.03 & 0.00 & $\begin{array}{l}0.00 \\
0.02\end{array}$ & 0.02 & 0.01 & 0.00 & 0.00 & $\begin{array}{l}0.14 \\
0.00\end{array}$ & 0.00 & 0.00 & 0.00 \\
\hline Sum cations & 4.01 & 4.00 & 4.02 & 4.01 & 4.00 & 3.99 & 4.05 & 4.02 & 4.03 & 4.02 & 3.98 & 3.98 & 3.99 & 3.99 \\
\hline $\mathrm{X}_{\mathrm{Mg}}$ & 0.91 & 0.90 & 0.90 & 0.90 & 0.92 & 0.92 & 0.89 & 0.96 & 0.93 & 0.90 & 0.92 & 0.90 & 0.92 & 0.92 \\
\hline $\mathrm{Al}^{\mathrm{IV}^{\mathrm{B}}}$ & 0.11 & 0.12 & 0.12 & 0.12 & 0.09 & 0.10 & 0.13 & 0.00 & 0.01 & 0.04 & 0.06 & 0.06 & 0.06 & 0.07 \\
\hline $\mathrm{Al}^{\mathrm{V}_{1}}$ & 0.02 & 0.01 & 0.03 & 0.02 & 0.02 & 0.03 & 0.00 & 0.00 & 0.00 & 0.00 & 0.03 & 0.04 & 0.02 & 0.02 \\
\hline Wollastonite & 0.42 & 0.42 & 0.36 & 0.42 & 0.40 & 0.39 & 0.37 & 0.50 & 0.47 & 0.48 & 0.07 & 0.03 & 0.03 & 0.03 \\
\hline Enstatite & 0.44 & 0.44 & 0.48 & 0.43 & 0.48 & 0.47 & 0.48 & 0.47 & 0.49 & 0.44 & 0.80 & 0.82 & 0.83 & 0.83 \\
\hline Ferrosilite & 0.04 & 0.05 & 0.05 & 0.05 & 0.04 & 0.04 & 0.06 & 0.02 & 0.04 & 0.05 & 0.07 & 0.08 & 0.07 & 0.07 \\
\hline
\end{tabular}

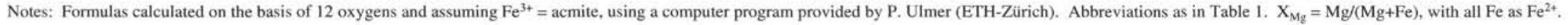


Table 4. Representative spinel compositions in Site 895 ultramafic and mafic lithologies.

\begin{tabular}{|c|c|c|c|c|c|c|c|}
\hline $\begin{array}{l}\text { Hole: } \\
\text { Core, section: } \\
\text { Interval }(\mathrm{cm}) \text { : } \\
\text { Piece: } \\
\text { Lithology: } \\
\text { Notes: }\end{array}$ & $\begin{array}{c}895 \mathrm{D} \\
4 \mathrm{R}-2 \\
126-129 \\
18 \\
\mathrm{HZ} \\
\text { core }\end{array}$ & $\begin{array}{c}895 \mathrm{D} \\
4 \mathrm{R}-2 \\
126-129 \\
18 \\
\mathrm{HZ} \\
\text { core }\end{array}$ & $\begin{array}{c}895 \mathrm{D} \\
4 \mathrm{R}-2 \\
126-129 \\
18 \\
\mathrm{HZ} \\
\text { altered rim* }\end{array}$ & $\begin{array}{c}895 \mathrm{D} \\
4 \mathrm{R}-2 \\
126-129 \\
18 \\
\mathrm{HZ} \\
\text { altered rim* }\end{array}$ & $\begin{array}{c}895 \mathrm{E} \\
1 \mathrm{R}-1 \\
83-87 \\
9 \\
\text { OLGB } \\
\text { core }\end{array}$ & $\begin{array}{c}895 \mathrm{E} \\
1 \mathrm{R}-1 \\
83-87 \\
9 \\
\text { OLGB } \\
\text { core }\end{array}$ & $\begin{array}{c}895 \mathrm{E} \\
2 \mathrm{R}-2 \\
6-10 \\
1 \\
\text { OLGB } \\
\text { core }\end{array}$ \\
\hline $\begin{array}{l}\mathrm{SiO}_{2} \\
\mathrm{TiO}_{2} \\
\mathrm{Al}_{2} \mathrm{O}_{3} \\
\mathrm{Cr}_{2} \mathrm{O}_{3} \\
\mathrm{Fe}_{2} \mathrm{O}_{3} \\
\mathrm{FeO} \\
\mathrm{MnO} \\
\mathrm{NiO} \\
\mathrm{MgO} \\
\mathrm{CaO} \\
\mathrm{Na}_{2} \mathrm{O} \\
\mathrm{K}_{2} \mathrm{O} \\
\mathrm{Cl} \\
\text { Total }\end{array}$ & $\begin{array}{r}0.00 \\
0.05 \\
24.99 \\
44.62 \\
2.42 \\
14.97 \\
0.23 \\
0.12 \\
13.75 \\
0.00 \\
0.01 \\
0.01 \\
0.01 \\
101.16\end{array}$ & $\begin{array}{r}0.00 \\
0.03 \\
26.03 \\
43.92 \\
2.37 \\
14.12 \\
0.21 \\
0.12 \\
14.35 \\
0.02 \\
0.03 \\
0.00 \\
0.01 \\
101.19\end{array}$ & $\begin{array}{r}6.13 \\
0.12 \\
1.80 \\
43.01 \\
12.08 \\
27.64 \\
0.93 \\
0.11 \\
7.09 \\
0.09 \\
0.07 \\
0.02 \\
0.04 \\
99.13\end{array}$ & $\begin{array}{r}7.45 \\
0.13 \\
2.34 \\
42.42 \\
8.85 \\
26.70 \\
0.93 \\
0.05 \\
8.61 \\
0.10 \\
0.08 \\
0.03 \\
0.04 \\
97.73\end{array}$ & $\begin{array}{r}0.00 \\
1.35 \\
22.03 \\
39.01 \\
6.58 \\
20.58 \\
0.47 \\
0.15 \\
10.18 \\
0.00 \\
0.00 \\
0.00 \\
0.01 \\
100.34\end{array}$ & $\begin{array}{r}0.00 \\
1.26 \\
21.28 \\
40.33 \\
6.44 \\
19.97 \\
0.43 \\
0.13 \\
10.41 \\
0.01 \\
0.02 \\
0.00 \\
0.01 \\
100.30\end{array}$ & $\begin{array}{r}0.00 \\
1.05 \\
17.67 \\
44.81 \\
5.06 \\
19.99 \\
0.37 \\
0.13 \\
9.68 \\
0.01 \\
0.00 \\
0.01 \\
\text { n.m. } \\
98.78\end{array}$ \\
\hline $\begin{array}{l}\mathrm{Si} \\
\mathrm{Ti} \\
\mathrm{Al} \\
\mathrm{Cr} \\
\mathrm{Fe}^{3+} \\
\mathrm{Fe}^{2+} \\
\mathrm{Mn} \\
\mathrm{Ni} \\
\mathrm{Mg} \\
\mathrm{Ca} \\
\mathrm{Na} \\
\mathrm{K} \\
\mathrm{Cl} \\
\text { Sum cations }\end{array}$ & $\begin{array}{l}0.00 \\
0.00 \\
0.88 \\
1.06 \\
0.05 \\
0.38 \\
0.01 \\
0.00 \\
0.62 \\
0.00 \\
0.00 \\
0.00 \\
0.00 \\
3.00\end{array}$ & $\begin{array}{l}0.00 \\
0.00 \\
0.91 \\
1.03 \\
0.05 \\
0.35 \\
0.01 \\
0.00 \\
0.64 \\
0.00 \\
0.00 \\
0.00 \\
0.00 \\
2.99\end{array}$ & $\begin{array}{l}0.21 \\
0.00 \\
0.07 \\
1.18 \\
0.32 \\
0.80 \\
0.03 \\
0.00 \\
0.37 \\
0.00 \\
0.00 \\
0.00 \\
0.00 \\
2.98\end{array}$ & $\begin{array}{l}0.26 \\
0.00 \\
0.09 \\
1.16 \\
0.23 \\
0.77 \\
0.03 \\
0.00 \\
0.44 \\
0.00 \\
0.01 \\
0.00 \\
0.00 \\
2.99\end{array}$ & $\begin{array}{l}0.00 \\
0.03 \\
0.81 \\
0.97 \\
0.15 \\
0.54 \\
0.01 \\
0.00 \\
0.48 \\
0.00 \\
0.00 \\
0.00 \\
0.00 \\
2.99\end{array}$ & $\begin{array}{l}0.00 \\
0.03 \\
0.79 \\
1.00 \\
0.15 \\
0.52 \\
0.01 \\
0.00 \\
0.49 \\
0.00 \\
0.00 \\
0.00 \\
0.00 \\
2.99\end{array}$ & $\begin{array}{l}0.00 \\
0.03 \\
0.68 \\
1.15 \\
0.12 \\
0.54 \\
0.01 \\
0.00 \\
0.47 \\
0.00 \\
0.00 \\
0.00 \\
\text { n.m. } \\
3.00\end{array}$ \\
\hline $\begin{array}{l}\mathrm{MgAl}_{2} \mathrm{O}_{4} \\
\mathrm{FeAl}_{2} \mathrm{O}_{4} \\
\mathrm{MnAl}_{2} \mathrm{O}_{4} \\
\mathrm{FeFe}_{2} \mathrm{O}_{4} \\
\mathrm{MgFe}_{2} \mathrm{O}_{4} \\
\mathrm{MnFe}_{2} \mathrm{O}_{4} \\
\mathrm{MgCr}_{2} \mathrm{O}_{4} \\
\mathrm{FeCr}_{2} \mathrm{O}_{4} \\
\mathrm{MnCr}_{2} \mathrm{O}_{4} \\
\mathrm{Fe}_{2} \mathrm{TiO}_{4}\end{array}$ & $\begin{array}{l}0.27 \\
0.17 \\
0.00 \\
0.02 \\
0.01 \\
0.00 \\
0.33 \\
0.20 \\
0.00 \\
0.00\end{array}$ & $\begin{array}{l}0.29 \\
0.16 \\
0.00 \\
0.02 \\
0.01 \\
0.00 \\
0.33 \\
0.18 \\
0.00 \\
0.00\end{array}$ & $\begin{array}{l}0.01 \\
0.03 \\
0.00 \\
0.06 \\
0.13 \\
0.01 \\
0.23 \\
0.50 \\
0.01 \\
0.00\end{array}$ & $\begin{array}{l}0.02 \\
0.04 \\
0.00 \\
0.06 \\
0.09 \\
0.00 \\
0.28 \\
0.48 \\
0.01 \\
0.00\end{array}$ & $\begin{array}{l}0.20 \\
0.20 \\
0.01 \\
0.04 \\
0.04 \\
0.00 \\
0.24 \\
0.24 \\
0.01 \\
0.03\end{array}$ & $\begin{array}{l}0.20 \\
0.19 \\
0.01 \\
0.04 \\
0.04 \\
0.00 \\
0.25 \\
0.24 \\
0.01 \\
0.03\end{array}$ & $\begin{array}{l}0.16 \\
0.17 \\
0.01 \\
0.03 \\
0.03 \\
0.00 \\
0.28 \\
0.29 \\
0.01 \\
0.02\end{array}$ \\
\hline $\begin{array}{l}\mathrm{xMg} \\
\mathrm{xFe}^{2+} \\
\mathrm{yAl} \\
\mathrm{yCr} \\
\mathrm{yFe}^{3+}\end{array}$ & $\begin{array}{l}0.61 \\
0.38 \\
0.44 \\
0.53 \\
0.03\end{array}$ & $\begin{array}{l}0.64 \\
0.35 \\
0.46 \\
0.52 \\
0.03\end{array}$ & $\begin{array}{l}0.30 \\
0.66 \\
0.05 \\
0.75 \\
0.20\end{array}$ & $\begin{array}{l}0.35 \\
0.61 \\
0.06 \\
0.78 \\
0.16\end{array}$ & $\begin{array}{l}0.45 \\
0.51 \\
0.42 \\
0.50 \\
0.08\end{array}$ & $\begin{array}{l}0.46 \\
0.50 \\
0.41 \\
0.52 \\
0.08\end{array}$ & $\begin{array}{l}0.45 \\
0.52 \\
0.35 \\
0.59 \\
0.06\end{array}$ \\
\hline
\end{tabular}

Notes: Formulas calculated using a computer program provided by $\mathrm{P}$. Ulmer (ETH-Zürich). ${ }^{*}=$ mixed measurements of optically undetectable spinel-chlorite intergrowths (see text); OLGB $=$ olivine gabbro; $\mathrm{HZ}=$ harzburgite; $\mathrm{n} . \mathrm{m} .=$ not measured; $\mathrm{xMg}=\mathrm{Mg}+\mathrm{Fe}_{\text {total }} ; \mathrm{xFe}^{2+}=\mathrm{Fe}^{2+} /\left(\mathrm{Fe}^{2+}+\mathrm{Mg}\right) ; \mathrm{yAl}=\mathrm{Al} /\left(\mathrm{Al}+\mathrm{Cr}+\mathrm{Fe}{ }^{3+}\right) ; \mathrm{yCr}=\mathrm{Cr} /(\mathrm{Cr}+\mathrm{Al}) ; \mathrm{yFe} e^{3+}=\mathrm{Fe}^{3+} /\left(\mathrm{Fe}^{3+}\right.$ $+\mathrm{Al}+\mathrm{Cr}$ ).

Vein- and veinlet-serpentine also display variable $\mathrm{Fe}_{\text {tot }}$ contents, from approximately 3 to $6 \mathrm{wt} \%$. Al contents are generally low (0 to $1 \mathrm{wt} \%$ $\mathrm{Al}_{2} \mathrm{O}_{3}$ ). Higher $\mathrm{Al}_{2} \mathrm{O}_{3}$ concentrations of up to $3 \mathrm{wt} \%$ (mostly observed in bastite pseudomorphs) can be discriminating and may indicate the presence of lizardite rather than chrysotile (Wicks and O'Hanley, 1988). In some cases, however, the presence of very fine, optically undetectable serpentine-chlorite intergrowths cannot be excluded. $\mathrm{Ni}$ contents in the serpentines vary from approximately 0.1 to about 0.4 $\mathrm{wt} \% \mathrm{NiO}$, with an average value of $0.1-0.2 \mathrm{wt} \%$ (Table 5). Chlorine contents are generally low $(<0.1-0.2 \mathrm{wt} \%)$, although very high chlorine contents (up to $6 \mathrm{wt} \%$ ) were measured in thin veinlets that were microscopically identified as brucite. The microprobe measurements yielded non-stoichiometric $\mathrm{Mg}$-rich compositions with highly variable Si-concentrations, suggesting micron-sized mixtures of serpentine and brucite.

The electron microprobe analyses of tremolite indicate that they are homogeneous in composition. With an average $\mathrm{X}_{\mathrm{Mg}}(=\mathrm{Mg} / \mathrm{Mg}+$ $\mathrm{Fe}_{\text {tot }}$ ) of approximately 0.94 and an $\mathrm{Al}^{\mathrm{IV}}$ range of $0.01-0.32$, they strictly plot in the tremolitic amphibole field defined by Hammarstrom and Zen (1986). Chlorite compositions are variable, with no clear relationship between the nature of the replacement and mineral composition. In particular, iron contents range from $5 \mathrm{wt} \% \mathrm{FeO}$ to approximately $13 \mathrm{wt} \% \mathrm{FeO}$. In the $\mathrm{Si}$ versus $\mathrm{Fe}^{2+} /\left(\mathrm{Mg}+\mathrm{Fe}^{2+}\right)$ classifi- cation scheme of Hey (1954), assuming that all iron present is divalent, the chlorite compositions scatter between the fields defined for penninite and clinochlore.

\section{Deformation and Veining in the Ultramafic Rocks}

High-temperature deformation fabrics of primary olivine, enstatite and spinel have been observed in the cored sections at Site 895. These fabrics are discussed in Gillis, Mével, Allan, et al. (1993) and in Kennedy et al. (1993), and have been related to an early deformation event under asthenospheric mantle conditions. In contrast, interstitial plagioclase impregnations and associated augite (often forming coarse-grained poikilitic crystals) are relatively undeformed, and may represent crystallization and reaction from a percolating mafic magma post-dating the high-temperature deformation in the ultramafic rocks but prior to serpentinization (Kennedy et al., 1993; Kennedy et al., this volume). Foliation is weakly defined in the dunites and harzburgites and is primarily associated with a preferred orientation of the primary spinel grains.

Deformation of the secondary phases in the ultramafic rocks recovered at Site 895 is generally minor. In particular, serpentine deformation is minimal and is limited to some bastite and vein serpentine occurrences, indicating that serpentinization in the recovered sec- 
Table 5. Representative serpentine compositions in Site 895 ultramafic and troctolitic lithologies.

\begin{tabular}{|c|c|c|c|c|c|c|c|}
\hline Hole: & $895 \mathrm{C}$ & 895D & 895D & 895D & 895D & 895D & 895D \\
\hline Core, section: & $4 \mathrm{R}-1$ & $4 \mathrm{R}-2$ & 4R-2 & $4 \mathrm{R}-2$ & $7 \mathrm{R}-1$ & $7 R-1$ & $7 R-1$ \\
\hline Interval $(\mathrm{cm})$ : & $52-54$ & $126-129$ & $126-129$ & $126-129$ & $81-84$ & $81-84$ & $81-84$ \\
\hline Piece: & ID & 18 & 18 & 18 & 13 & 13 & 13 \\
\hline Lithology: & IDU & $\mathrm{HZ}$ & $\mathrm{HZ}$ & $\mathrm{HZ}$ & TRO & TRO & TRO \\
\hline Association: & vein & after opx & after opx & after ol & after ol & after ol & after ol \\
\hline $\mathrm{SiO}_{2}$ & 41.58 & 43.92 & 42.16 & 43.71 & 39.67 & 43.79 & 42.98 \\
\hline $\mathrm{TiO}_{2}$ & 0.03 & 0.00 & 0.00 & 0.02 & 0.01 & 0.04 & 0.00 \\
\hline $\mathrm{Al}_{2} \mathrm{O}_{3}$ & 1.51 & 0.57 & 0.97 & 0.22 & 1.93 & 0.70 & 0.48 \\
\hline $\mathrm{Cr}_{2} \mathrm{O}_{3}$ & 0.18 & 0.02 & 0.00 & 0.00 & 0.00 & 0.02 & 0.00 \\
\hline $\mathrm{FeO}$ & 3.85 & 3.51 & 4.43 & 3.85 & 10.55 & 3.18 & 4.10 \\
\hline $\mathrm{MnO}$ & 0.12 & 0.05 & 0.04 & 0.07 & 0.11 & 0.02 & 0.05 \\
\hline $\mathrm{NiO}$ & 0.06 & 0.11 & 0.17 & 0.20 & 0.35 & 0.17 & 0.15 \\
\hline $\mathrm{MgO}$ & 39.19 & 39.22 & 39.73 & 39.98 & 34.91 & 40.20 & 39.89 \\
\hline $\mathrm{CaO}$ & 0.41 & 0.00 & 0.01 & 0.01 & 0.26 & 0.12 & 0.13 \\
\hline $\mathrm{Na}_{2} \mathrm{O}$ & 0.05 & 0.01 & 0.02 & 0.00 & 0.01 & 0.04 & 0.03 \\
\hline $\mathrm{K}_{2} \mathrm{O}$ & 0.02 & 0.01 & 0.01 & 0.01 & 0.02 & 0.00 & 0.02 \\
\hline $\mathrm{Cl}$ & 0.07 & 0.04 & 0.09 & 0.08 & 0.12 & 0.05 & 0.07 \\
\hline $\mathrm{H}_{2} \mathrm{O}$ & 12.69 & 12.88 & 12.75 & 12.91 & 12.33 & 12.99 & 12.84 \\
\hline Total & 99.77 & 100.34 & 100.36 & 101.04 & 100.24 & 101.32 & 100.72 \\
\hline $\mathrm{Si}$ & 1.96 & 2.04 & 1.98 & 2.03 & 1.93 & 2.02 & 2.01 \\
\hline $\mathrm{Ti}$ & 0.00 & 0.00 & 0.00 & 0.00 & 0.00 & 0.00 & 0.00 \\
\hline $\mathrm{Al}$ & 0.08 & 0.03 & 0.05 & 0.01 & 0.11 & 0.04 & 0.03 \\
\hline $\mathrm{Cr}$ & 0.01 & 0.00 & 0.00 & 0.00 & 0.00 & 0.00 & 0.00 \\
\hline $\mathrm{Fe}^{2+}$ & 0.15 & 0.14 & 0.17 & 0.15 & 0.43 & 0.12 & 0.16 \\
\hline Mn & 0.01 & 0.00 & 0.00 & 0.00 & 0.00 & 0.00 & 0.00 \\
\hline $\mathrm{Ni}$ & 0.00 & 0.00 & 0.01 & 0.01 & 0.01 & 0.01 & 0.01 \\
\hline $\mathrm{Mg}$ & 2.76 & 2.72 & 2.78 & 2.76 & 2.52 & 2.76 & 2.77 \\
\hline $\mathrm{Ca}$ & 0.02 & 0.00 & 0.00 & 0.00 & 0.01 & 0.00 & 0.01 \\
\hline $\mathrm{Cl}$ & 0.01 & 0.00 & 0.01 & 0.01 & 0.01 & 0.00 & 0.01 \\
\hline $\mathrm{H}$ & 3.99 & 4.00 & 3.99 & 3.99 & 3.99 & 4.00 & 3.99 \\
\hline Sum cations & 5.00 & 4.93 & 5.00 & 4.97 & 5.02 & 4.95 & 5.00 \\
\hline
\end{tabular}

Notes: Cation distribution calculated on the basis of 9 oxygens (total $\mathrm{Fe}$ as $\mathrm{FeO}$ ), using a computer program provided by P. Ulmer (ETH-Zürich). Abbreviations are as in Table 1.

tions occurred under static conditions. Serpentine replacement textures are predominantly pseudomorphic, which excludes a considerable amount of serpentine recrystallization. Brittle deformation is dominated by rare pull-apart structures and fracture-fillings in primary and secondary phases. Kink bands in some bastite-serpentine and weakly deformed secondary minerals such as chlorite and tremolite are locally observed, whereby slight folding and shearing of the veins is rare. A preferred orientation of the microfabrics is generally not detectable on a thin-section scale, which suggests that the various deformation features are not related to geometrically well-defined tectonic stress phases. Although on a small scale, sets of subparallel veins have been observed, most of the veins and veinlets are randomly oriented. The tensional components of deformation seem to be related to fluid penetration paths and rock rheology rather than to largescale tectonic events.

Multiple generations of macroscopic veins and veinlets crosscut the Site 895 ultramafic rocks. As discussed above, the pervasive background alteration is dominated by mesh-textured lizardite and/or chrysotile replacement after olivine surrounded by anastomosing networks of thin antigorite \pm magnetite \pm clay microscopic veinlets. Macroscopic crosscutting relationships could be defined on board ship by observation of the complete cored sections (Gillis, Mével, Allan, et al., 1993). Six different vein types were distinguished, and generally occur at both a macroscopic and microscopic scale. These vein types are briefly summarized here:

1. Early, rare, greenish to gray tremolite $+\mathrm{Mg}$-chlorite \pm serpentine veins (only in harzburgites)

2. Greenish blue serpentine \pm magnetite \pm chlorite veins

3 . White serpentine + magnetite \pm chlorite veins

4. White, thin serpentine \pm magnetite \pm brucite/talc \pm clay veins

5 . White serpentine \pm magnetite \pm clay \pm zeolite veins

6. Late, white aragonite veins

Type 1 tremolite + chlorite \pm serpentine veins are rare and have only been observed in the harzburgites. Crosscutting relationships and assemblages suggest that they represent an early stage of rock alteration, and may be associated with the characteristic replacement of many orthopyroxene grains by tremolite. Vein-tremolite forms elongate, subparallel to radiating crystals. Varying proportions of chlorite and serpentine form the vein walls. The veins can locally become thicker or branching, and include orthopyroxene grains completely pseudomorphed by tremolite.

Relatively thick (to $0.5-1 \mathrm{~cm}$ across) lizardite and/or chrysotile \pm magnetite veins of Type 2 are common in the dunites and harzburgites (Fig. 3). They occasionally show a color zonation, with a greenish central zone composed of fine-grained fibrous antigorite with interstitial chrysotile (generally devoid of magnetite), bordered by a thin dark-blue rim composed of serpentine + magnetite + chlorite. In other cases, the veins are monomineralic, composed of coarser grained, light green, weakly deformed antigorite(?) fillings with a typical crack-seal texture, or long, undeformed, obliquely inclined asbestos-like chrysotile fibers. Secondary diopside occasionally forms elongated acicular crystals subperpendicular to the vein walls.

The Type 2 veins are often subperpendicularly cut by thin $(<1$ $\mathrm{mm}$ ) subparallel, white brucite + serpentine vein sets of Type 4 (Fig. $3)$. These are white and rarely monomineralic, with a typical wavy morphology. They can accommodate considerable amounts of chlorine (up to $6 \mathrm{wt} \%$ ). Such high chlorine contents have been detected only in this vein type; the chlorine concentration in the other veins is otherwise very low. Brucite never coexists with talc; whereby talc is limited to orthopyroxene alteration (Pl. 1, Fig. 1). Brucite typically forms thin bands associated with linear fine-grained magnetite enrichments. Type 2 veins occasionally represent a preferential pathway for later penetration of microcrystalline carbonate material, forming irregular, very thin anastomosing veins.

Veins of Type 3 are mineralogically homogeneous. Lizardite and/ or chrysotile is the principal mineral phase, whereas magnetite and chlorite predominantly occur in variable but minor amounts. The veins are white, thin (to $1 \mathrm{~mm}$ ) and mostly straight.

Late, white aragonite veins of Type 6 represent the latest veining phase in the ultramafic rocks. Crystallization temperatures of aragonite, estimated on the basis of oxygen isotope ratios (discussed below), are very low (less than $10^{\circ} \mathrm{C}$ ). Aragonite forms elongate to radiating fibrous patches, locally accompanied by clasts of the host rock. Aragonite crystal morphology suggests that they formed in open cracks. Irregular thin infiltration of carbonate material in the serpentine-olivine groundmass, and fibrous tremolite ( \pm chlorite) enrichments are generally associated with the main carbonate vein.

\section{Troctolitic and Gabbroic Rocks}

Gabbro, olivine gabbro, and troctolite occur in the lower part of Hole 895D (Cores 147-895D-6R through 9R) and are particularly abundant in Hole 895E. The gabbroic rocks at Site 895 are closely associated with dunites and possibly form cyclic intervals. The occurrence of dunite with impregnations of plagioclase and clinopyroxene, grading into troctolitic and olivine gabbro compositions is particularly spectacular in Core 147-895C-4R. The association of dunite with refractory harzburgite may be characteristic of the Hess Deep upper mantle. The petrology and textures of the ultramafic and mafic rocks, as well as the relative abundance of dunites and their close affinity to the gabbroic rocks, suggest that these sequences are products of melt migration, wall-rock reaction and cumulus olivine crystallization close to the mantle/crust boundary (Gillis, Mével, Allan, et al., 1993; Dick et al., 1993; Mével et al., 1993; Allan and Dick, this volume; Arai and Matsukage, this volume; Dick and Natland, this volume).

\section{Alteration of Troctolitic and Gabbroic Rocks}

The Site 895 troctolitic and gabbroic rocks investigated in this study are characterized by a large variation in grain size, modal composition, and degree of alteration and deformation (see Table 1; Fig. 4). The rocks are moderately to pervasively altered and typically ex- 


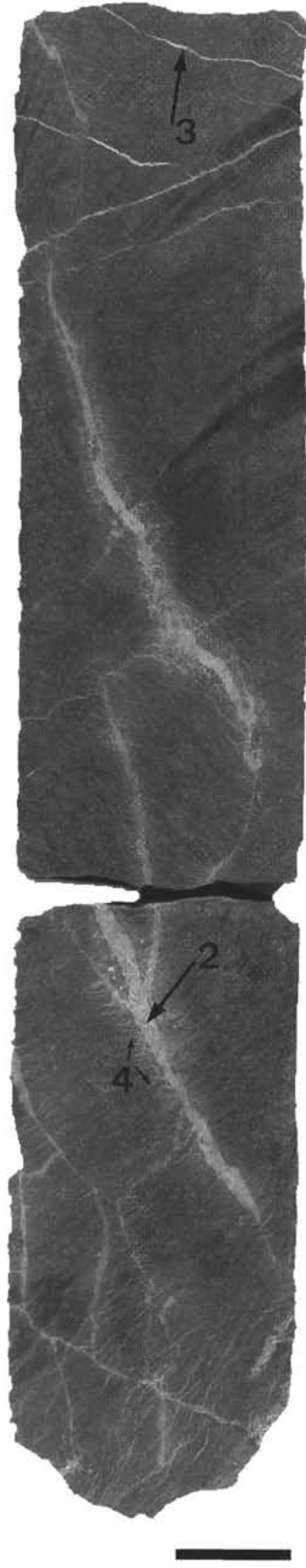

Figure 3. Distinct vein generations in a Site 895 dunite Sample 147-895E6R-4 (Piece 2A and 2B, 37-65 cm). Relatively thick, greenish blue serpentine \pm magnetite \pm chlorite veins $(2)$ are cut by thin wavy serpentine \pm magnetite \pm brucite \pm clay veinlets (4). White, nearly straight serpentine + magnetite \pm chlorite veins are visible in the upper part of the sample (3). Numbers refer to vein types discussed in the text. Scale bar is $2 \mathrm{~cm}$. hibit multiple phases of overprinting and cataclastic deformation (Mével and Stamoudi, this volume). Static alteration is dominant, producing pseudomorph textures after the primary phases and distinct, well-developed coronas of chlorite between olivine and plagioclase grains (Pl. 2, Figs. 1-3).

\section{Olivine}

Primary olivine is moderately to pervasively altered, but is locally well preserved in some troctolite and olivine gabbro samples (Table 1). The abundance of olivine varies from 40 modal $\%$ to greater than $85 \%$ in the troctolites and plagioclase-impregnated dunites to $<1 \%$ in the gabbros. Olivine grain boundaries are commonly embayed and exhibit complex, heterogeneous, and zoned replacement textures. Alteration products include mesh-textured serpentine, magnetite, chlorite, clay, tremolite, and less commonly, secondary diopside, talc, and rare cummingtonite. Chlorite can be finely intergrown with serpentine or mixed-layered clays. Replacement of olivine by serpentine is more prevalent in the troctolites and plagioclase-impregnated dunites than in the olivine gabbros or gabbros. Distinct coronas of colorless chlorite as reaction products of plagioclase and olivine occur throughout the gabbroic and troctolitic rocks.

In the troctolites and plagioclase-impregnated dunites, relict olivine occurs as clusters of small (generally less than $1 \mathrm{~mm}$ ) subangular to subrounded kernels separated by anastomosing veinlets of meshtextured lizardite/chrysotile, light green Fe-chlorite, fine-grained secondary magnetite, and in some samples, clay. Very fine intergrowths (on a $\mu \mathrm{m}$-scale) of serpentine and chlorite have been identified by scanning electron microscope backscatter and element distribution scanning across altered olivine grains. The grain sizes and morphologies of the original olivine grains are marked by a continuous optical extinction of the olivine clusters, together with distinct mineral zonation textures and the well-defined coronas of translucent chlorite ( $\mathrm{Pl}$. 2, Fig. 1) The zoned replacement of olivine is characterized by thin rims $(10-30 \mu \mathrm{m})$ of fine-grained diopsidic clinopyroxene at the contact to the chlorite coronas, grading into $0.5-1$-mm-thick turbid clusters of radiating fibrous tremolite, followed by light green chlorite towards the serpentinized cores. The diopsidic rims may represent primary interstitial clinopyroxene, now altered to secondary clinopyroxene. Locally, discontinuous microfractures filled with very fine-grained, turbid fibrous tremolite crosscut the mesh-serpentine textures. In highly altered troctolites and plagioclase impregnated dunites, lower temperature overprinting, commonly associated with late-stage veining (see below) and cataclastic deformation, is marked by an increase in the modal proportions of Fe-rich chlorite intergrown with serpentine, clays and fine-grained magnetite.

In the olivine gabbros and gabbros, subangular to subrounded olivine grains, up to $2 \mathrm{~mm}$ across, commonly form clusters with cumulate textures. These clusters are variably altered to radiating acicular tremolite intergrown with colorless chlorite and, less commonly, fine-grained, turbid granular diopside ( \pm oxides) or talc. As in the troctolites, 10-30 $\mu \mathrm{m}$-sized rims of secondary clinopyroxene may mark the boundary to translucent chlorite coronas that have formed between the original cumulate olivine and plagioclase grains (PI. 2, Figs. 2, 3). In more-altered, veined, and deformed samples, the original olivine grains are completely pseudomorphed by irregularly shaped, turbid patches of fibrous tremolite and chlorite ( \pm diopside \pm talc), or serpentine, clays, and iron oxides.

\section{Plagioclase}

Plagioclase and its alteration products make up 40 to 50 modal\% of the olivine gabbros and gabbros, and varies from approximately $5 \%$ up to $80 \%$ in the troctolites. Plagioclase alteration is typically heterogeneous and varies from $<10 \%$ to $100 \%$. In the least-altered samples, plagioclase forms relatively clear, subangular to subrounded crystals with well-defined polysynthetic twinning and cleavage planes. Grain boundaries are often embayed and are marked by incipient alteration to light green, weakly pleochroic $\mathrm{Al}$-rich chlorite that 

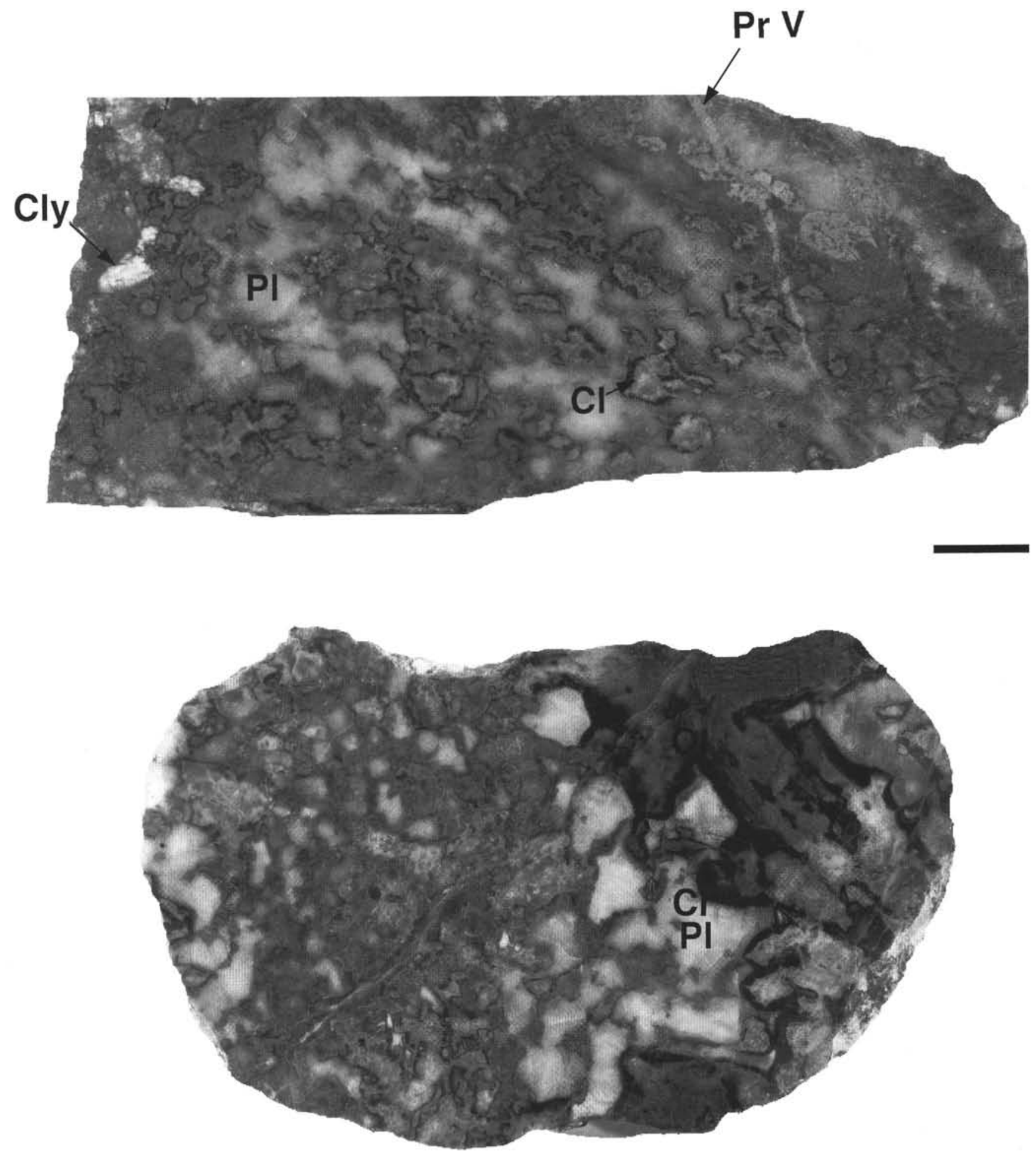

Figure 4. Photograph showing variations in mineral modes, grain sizes, and alteration textures over a centimeter scale in troctolites. Dark green chlorite (Cl) typically forms up to 1 -mm-thick coronas around olivine (OI) grains in contact with plagioclase (PI). Replacement of olivine by fibrous tremolite and plagioclase by anorthite, prehnite, hydrogrossular, or clays (Cly) is heterogeneous (see Pl. 2, Figs. 2, 3). Crosscutting veins are filled with prehnite (Pr V). (Samples 147895D-8R-2, Pieces 3 and $4,20-41 \mathrm{~cm}$ ). Scale bars are $1 \mathrm{~cm}$. 
forms a thin $(10-50 \mu \mathrm{m})$ seam between the plagioclase grains and the coronas of colorless chlorite. Formation of fine-grained, light green chlorite, colorless fibrous tremolite \pm clinopyroxene \pm epidote and rarely prehnite along cleavage planes and grain boundaries, as well as the local patchy occurrence of secondary anorthitic plagioclase, are related to the presence of subparallel sets of conjugate microfractures and micro-veinlets, giving the plagioclase grains an increasingly turbid appearance. The veinlets are commonly discontinuous and crosscut as well as parallel cleavage planes. An increase in the density of microfractures and veinlets is associated with incipient brecciation of the plagioclase grains and replacement by fine-grained mats of fibrous prehnite \pm chlorite, and patches of very fine-grained to microcrystalline hydrogrossular, zeolites (predominantly thomsonite and tetranatrolite), white mica, mixed-layered smectite/chlorite, kaolinite, boehmite (an Al-hydroxide), iron oxides, and iron hydroxides ( $\mathrm{Pl}$. 2, Fig. 1). In pervasively altered and intensively deformed samples, individual plagioclase grain boundaries become indistinguishable and are completely replaced by the calc-silicate and Al-rich phases.

Microscopically, the hydrogrossular-type replacement of plagioclase is characterized by cloudy, dark, reddish brown to nearly black isotopic patches, commonly with poorly defined crystal form and grain boundaries. Clear, fine-grained aggregates of isotropic, granular crystals occur locally. Microprobe analysis combined with XRD determinations confirm the presence of the calc-silicate alteration products and allow a better characterization of their compositions (see below, and Gillis, Mével, Allan, et al., 1993). Boehmite and thomsonite have been identified by XRD and electron microprobe analysis, whereas kaolinite and tetranatrolite as alteration products of plagioclase could only be identified through XRD determinations. The term hydrogrossular is used here to collectively describe the finegrained, granular, high-relief isotopic crystals as well as the more common reddish brown, microcrystalline alteration products. Microprobe measurements indicate a spectrum of compositions of these phases, transitional from prehnite to stoichiometric hydrous garnet. The textural and chemical characteristics of these phases are identical to those described by Honnorez and Kirst (1975) from rodingites dredged along mid-Atlantic fracture zones. These authors tentatively called the transitional phases "paragarnet." Although hydrogrossular and its transitional phases are observed as an alteration product of plagioclase in all the gabbroic and troctolitic lithologies, prehnite is more abundant in the olivine gabbros and gabbros, and is commonly associated with the presence of discrete prehnite or zeolite veins.

\section{Clinopyroxene}

Primary clinopyroxene contents are also heterogeneous, varying from $<1$ to approximately 70 modal\% locally, and primarily occur as fine-medium to very coarse (pegmatitic) grains in the gabbros (e.g., in Sections 147-895D-7R-1 and 147-895E-4R-3). The occurrence of single clinopyroxene (cpx) crystals, up to $12 \mathrm{~cm}$ long, is particularly characteristic of Section 147-895E-4R-3. In the troctolitic and olivine gabbros, augitic clinopyroxene locally forms fine-grained coronas between olivine and plagioclase grains or occurs as fine- to mediumgrained intergranular anhedral grains.

Augitic to diopsidic clinopyroxene in the gabbros is characteristically coarse-grained to pegmatitic. In hand sample, the large pyroxenes are olive green in color and have a classic diallage morphology, with a fine wavy cleavage that imparts a distinct, reflective, satiny sheen. Microscopically, the crystals typically have $\{100\}$ partings altered to turbid fine-grained material and may have orthopyroxene exsolution lamellae now replaced by chlorite. Fine-grained, colorless tremolite, chlorite, secondary diopside, and prehnite \pm epidote occur between the partings, in pull-apart microfractures, and as thin $(<50 \mu \mathrm{m})$, discontinuous veinlets crosscutting the relict pyroxene grains (Pl. 2, Fig. 4). Locally the pyroxenes are cut by thin, discontinuous veinlets filled with dark fine-grained, nearly isotropic granular crystals, possibly consisting of microcrystalline mixtures of hydrogrossular (or epidote) and iron oxides. The pyroxene grains are commonly bent and cracked, and exhibit deformation and kink bands, with undulatory and wavy extinction. Veining predates deformation as indicated by offset relationships and folding of the veins parallel to the undulations and kink bands in the host pyroxene crystals. Grain boundaries are often embayed and are either sharp or have rims $(<20 \mu \mathrm{m}$ to $1 \mathrm{~mm}$ ) of colorless chlorite or radiating clusters of tremolite. Clear secondary diopside occurs in pull-apart microstructures, or locally as patches in the relict pyroxene grains and at the grain boundaries. These clear diopsides contain primary and secondary vapor-dominated fluid inclusions that are oriented parallel to cleavage planes (Gillis, Mével, Allan, et al., 1993). Coarser grained tremolite crystals locally form ragged, discontinuous rims, up to 1 mm long, at the edges of the grains (PI. 2, Fig. 5) and characteristically have undulatory extinction that is oblique to the original pyroxene grains.

\section{Orthopyroxene}

Orthopyroxene could not unequivocally be identified in the samples investigated in this study. However, rare irregular patches completely altered to chlorite and tremolite observed in some gabbro samples may represent pseudomorphs of original orthopyroxene. Shipboard studies indicated that alteration of the orthopyroxene is heterogeneous and is dominated by the replacement by cummingtonite, tremolite, chlorite, and fine-grained oxides (Gillis, Mével, Allan, et al., 1993). Cummingtonite may rarely be associated with talc.

\section{Oxides}

The abundance of primary spinel in the troctolitic and gabbroic rocks is highly variable, ranging from up to $3 \%$ locally in impregnated dunites to a virtual absence in some gabbros. Magnetite is the most common secondary oxide and occurs as a replacement product of primary spinel and olivine. Fine-grained secondary magnetite typically occurs as thin, anastomosing veinlets together with mesh-textured serpentine in altered olivine grains in the troctolites and impregnated dunites. In rare metagabbro samples, magnetite grains, up to $1 \mathrm{~mm}$ in size, may be cracked and exhibit pressure shadows of chlorite with anomalous blue interference colors (Pl. 3, Fig. 3).

\section{Deformation in the Troctolitic and Gabbroic Rock}

\section{Microfabrics}

Static metamorphism is predominant in the mafic rocks, resulting in pseudomorphic replacement and coronitic textures. Penetrative deformation has produced a weak to well-developed foliation, defined by the elongation and alignment of irregularly shaped grains of plagioclase with chlorite coronas, clinopyroxene, and clusters of tremolite + chlorite + secondary clinopyroxene as pseudomorphs of the original olivine (Kennedy et al., this volume). Although the degree of alteration and deformation is highly variable, relicts of the primary phases, as well as their pseudomorphing replacement products, invariably exhibit extensive undulatory extinction, and clinopyroxene grains typically have well-developed kink bands.

Local penetrative shear zones in the olivine gabbros (Pl. 3, Figs. 1,2 ) and gabbros as well as fine-grained, gray to white, sheared metagabbroic rocks surrounded by serpentinized dunite (Fig. 5) were observed in several samples (e.g., in Samples 147-895C-4R-1, Piece 7; 147-895D-8R-2, Piece 19; 147-895D-10R-1, Piece 8; 147-895E-1R1, Piece 9, 147-895E-4R-2, Piece 17; 147-895E-6R-1, Piece 14; and 147-895E-8R-4, Piece 9). The sheared metagabbros were only recovered as small, pebble-sized pieces (up to $5 \mathrm{~cm}$ ) and consist primarily of multiple generations of fine-grained tremolite, chlorite, and locally relict clinopyroxene, aligned in parallel shear bands and exhibiting a continuous extinction parallel to shearing. Synkinematic replacement of primary clinopyroxene by tremolitic amphibole is particularly well observed in Sample 147-895E-6R-1 (Piece 14, 78-80 cm; Pl. 3, Fig. 


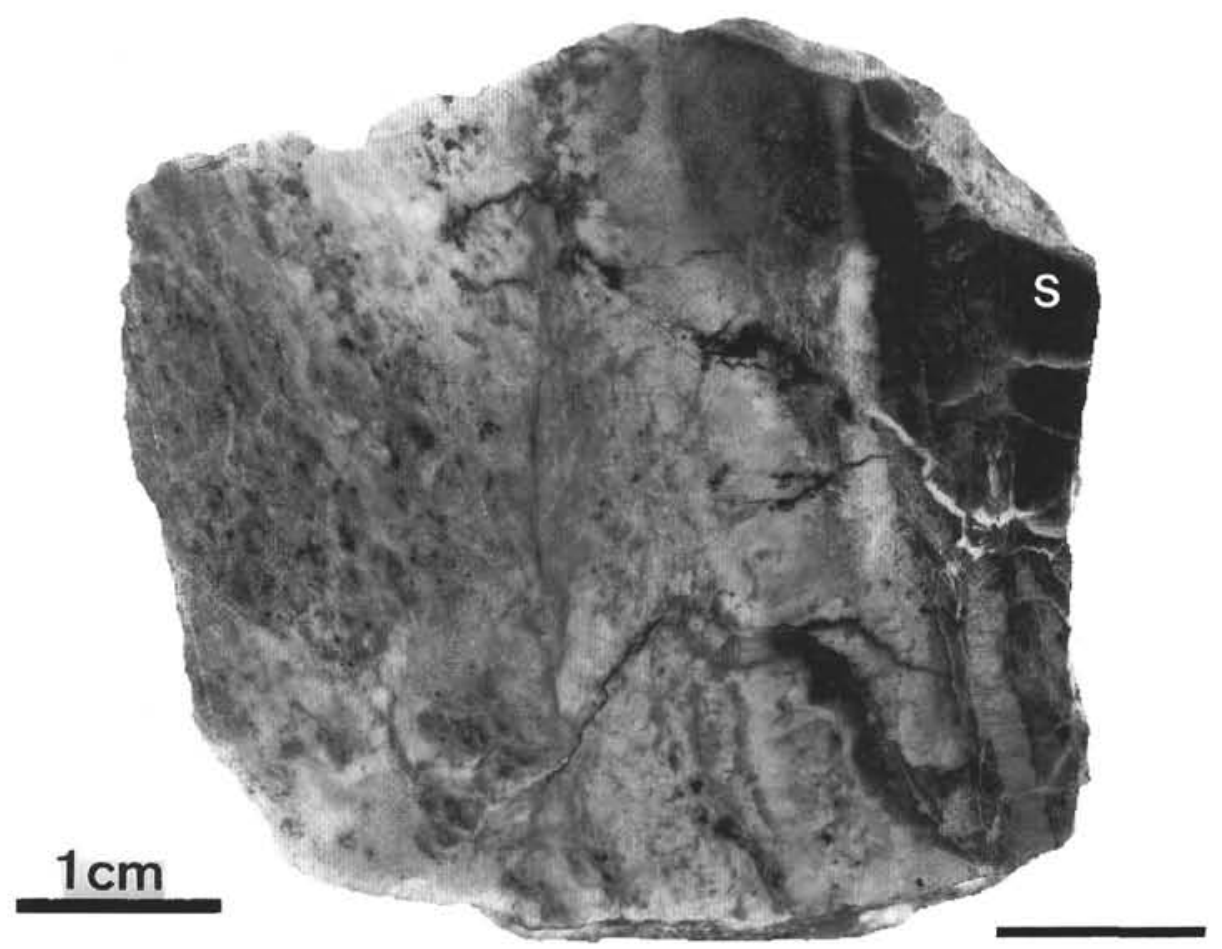

Figure 5. Sheared metagabbros sample with contact to completely serpentinized dunite (S). Pervasive alteration to fine-grained, fibrous tremolitic amphibole and chlorite defines the foliation (Sample 147-895D-8R-2, Piece 19, 145-151 cm; Pl. 3, Fig. 3). Scale bar is $1 \mathrm{~cm}$.

3). Clinopyroxene occurs as small ( $<50 \mu \mathrm{m}$ long) elongated grains with diffuse grain boundaries and is replaced by fine-grained, light brown, slightly pleochroic amphibole that forms wavy tails, up to 5 $\mathrm{mm}$ long, together with chlorite. In less-sheared regions of the sample, tremolite and chlorite form elongated, subparallel clusters that are reminiscent of the corona textures in less-deformed olivine gabbros. Subsequent veining is evident by crosscutting relationships between veins and the foliation created by shearing. Post-vein deformation has resulted in undulatory extinction, well-developed kink bands and microfolding in the foliated chlorite + tremolite groundmass as well as in the crosscutting chlorite veins. The similarity of the mineral assemblage chlorite + tremolite with mineral assemblages in the lessaltered olivine gabbros, as well as local preserved contacts to serpentinized dunite, suggest that the metagabbros represent sheared dikelets of the gabbroic rocks. Although the sheared metagabbros are macroscopically similar to rodingites recovered from the mid-Atlantic and those observed in ophiolite complexes, the distinct lack of any of the calc-silicate phases characteristic of rodingites (i.e., prehnite, epidote, hydrogrossular, idocrase; not identified in XRD analysis) indicates that these rocks are not a product of $\mathrm{Ca}$-metasomatism, but are the result of localized deformation and fluid infiltration under greenschist-facies conditions that is characteristic of the background alteration in the mafic rocks.

In the weakly to moderately altered gabbroic samples, local shearing has produced the elongation and alignment of chlorite, tremolite, and secondary diopsidic clinopyroxene after primary olivine, and has also led to the new formation of very fine-grained, granular secondary diopside in thin stringers oriented parallel to the shear zone (Pl. 3 , Figs. 1,2). Away from the shear zones, olivine grains are statically altered to yellow-green, pleochroic (Fe-rich) chlorite and tremolite. These features, as well as the presence of unoriented fibrous tremolite clusters directly bordering the shear zones, indicate that greenschistfacies alteration and hydration was pre-, syn-, and postkinematic.

In highly altered samples, strain partitioning has produced complex fabrics, with more penetrative deformation in highly altered zones dominated by hydrous minerals and cataclastic deformation in less-altered zones with more abundant medium- to coarse-grained clinopyroxene and/or plagioclase. High strain deformation is concentrated in local shear zones and is characterized by shear bands of chlorite and tremolite, deformation twins in plagioclase and local folding of chlorite, tremolite, and early microvein generations. For example, in a 1-cm-thick shear zone in the meta-olivine gabbro Sample 147-895E-4R-2 (Piece 17, 139-141 cm), light green chlorite with stringers of very fine-grained tremolite and secondary oxides forms $\mathrm{mm}$-thick shear bands, separated by highly elongated thinner $(<0.5$ $\mathrm{mm}$ ) bands of turbid, brownish tremolite and secondary oxides (Pl. 3, Fig. 4). The tremolite stringers in the chlorite form steep angles to the shear planes. Directly away from the shear zone, irregularly sized clasts representing the original chlorite coronas, the tremolite and secondary clinopyroxene pseudomorphs after olivine, and plagioclase completely pseudomorphed by prehnite are highly elongated and oriented subparallel to the shear zone. Gradation to more brittle fracturing and brecciation of completely altered turbid plagioclase occurs within $1 \mathrm{~cm}$ of the shear zone in coarser grained portions of the sample.

Strain partitioning in some coarse-grained gabbros is characterized by the presence of $0.5-1 \mathrm{~mm}$ thin bands of highly elongated chlorite and tremolite between bent, cracked and locally imbricated plagioclase and clinopyroxene grains. Multiple phases of deformation and veining has produced displacement along the $\{100\}$ partings and microfolding of individual relict clinopyroxene lamellae (Pl. 2, Fig. 6) with chlorite, and has resulted in deplacement of shear bands, strong undulatory extinction in most phases, and prehnite veinlets.

\section{Veins}

Veins in the troctolitic and gabbroic rocks vary from thin $(<1$ $\mathrm{mm}$ ), discontinuous, filled microfractures to discrete networks of veins up to $1 \mathrm{~cm}$ thick. Filled microfractures are most common and occur in all lithologies. They typically form sinuous, anastomosing or overlapping, subparallel fracture sets and exhibit large variations in 
Table 6. Representative plagioclase compositions in Site 895 ultramafic and mafic lithologies.

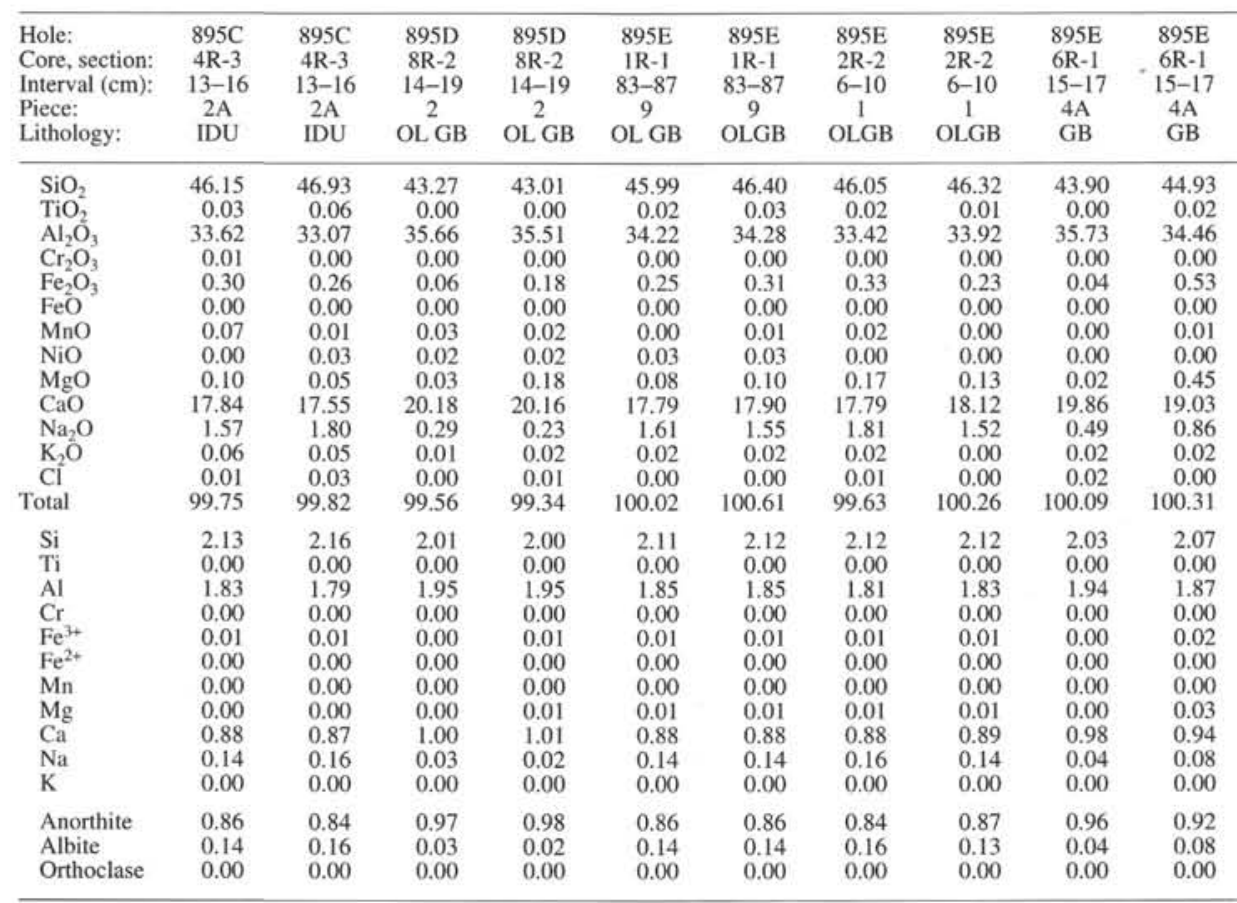

Note: Cation proportions calculated on the basis of 5 cations and 16 charges. Abbreviations as in Table 1.

thickness, length, mineralogies, and degree of subsequent deformation. Mineralogies include chlorite, tremolite, diopside, secondary plagioclase, talc, prehnite, zeolite, clays, serpentine, magnetite, and rare epidote and calcite. Although the microveins are typically monomineralic, composite veins and veinlets are locally observed. In addition, the mineralogies may change, depending on the host mineral the veinlet is cutting; and multiple, crosscutting fracture-filling phases are common. Subsequent deformation, resulting in vein offset, folding, and undulatory extinction in the vein-filling phases, is also evident in some samples.

The troctolites are commonly cut by multiple generations of thin $(10-50 \mu \mathrm{m})$, anastomosing veinlets in microfractures. The earliest phases include discontinuous veinlets of mesh-textured serpentine (antigorite?), light green Fe-chlorite, and fine-grained secondary magnetite, crosscutting olivine grains, as well as rare microveins of secondary diopside \pm tremolite \pm chlorite. These are crosscut by variably oriented fractures filled by chlorite, serpentine, clay and hydrogrossular. Zoned veinlets (up to $50 \mu \mathrm{m}$ thick) constitute the latest fracture-filling phase and crosscut the earlier microvein generations, the zoned replacement textures in relict olivines, and the interstitial, hydrogrossular-altered plagioclase. These composite veins consist of chlorite along the walls with light greenish brown, low birefringent serpentine (lizardite?), mixed-layered smectite/chlorite clays, or very fine-grained magnetite + granular hydrogrossular(?) in the centers.

The earliest generation of microfractures in the olivine gabbros and gabbros are generally monomineralic and filled with secondary diopside, tremolite, or chlorite. Incipient brecciation of plagioclase as well as the formation of cracks and kink bands in clinopyroxene are associated with an increase in brittle deformation and density of subparallel, locally conjugate microfractures with variable mineral infillings. An abundance of microfractures and extensive alteration in the coarse-grained olivine gabbros and gabbros are also associated with discrete, irregular veins of prehnite \pm hydrogrossular, chlorite, or thomsonite. Prehnite veins have characteristic boytrioidal infillings, consisting of clear, radiating blades of prehnite in the centers and red- dish brown hydrogrossular phases along the rims of the spherulites (Pl. 3, Figs. 5, 6). These textures are identical to those in prehnite veins observed from fracture zones in the equatorial Mid-Atlantic Ridge (Honnorez and Kirst, 1975). Multiple opening and filling events may be evident by thin $(<10 \mu \mathrm{m})$, wavy seams of chlorite and clays in the centers of the veins. In some samples, a well-defined foliation of chlorite + tremolite clusters and sheared, light green chlorite clasts in the immediate vicinity of the prehnite veins, in conjunction with the lack of an oriented fabric in the vein-filling minerals, suggests that the penetration of hydrothermal fluids was enhanced by the presence of a previous shear zone.

\section{Mineral Chemistry of Primary and Secondary Phases}

The relict olivine in the olivine gabbros and troctolitic rocks have relatively constant compositions, with $88-89 \mathrm{~mol} \%$ forsterite. $\mathrm{Ni}$ contents range from 0.29 to $0.41 \mathrm{wt} \%$ and are similar or only slightly lower than those in the surrounding harzburgites and dunites (Table 2). In general, the secondary phases after olivine show little variation between different rock types or degrees of alteration. Plagioclase compositions are constant within an individual sample and $\mathrm{Ca}$-rich, with anorthite (An) contents ranging from $\mathrm{An}_{85}$ in the least altered samples to $\mathrm{An}_{98}$ in highly altered gabbros (Table 6). An increase in the anorthite component is clearly related to a higher degree of alteration and deformation, and is commonly associated with the presence of discrete veins filled with prehnite \pm hydrogrossular phases.

Primary clinopyroxene in the coarse-grained gabbros are diopsidic to augitic in composition and exhibit relatively constant $\mathrm{X}_{\mathrm{Mg}}$ (= $\left.\mathrm{Mg} /\left(\mathrm{Mg}+\mathrm{Fe}_{\text {total }}\right)\right)$ ranging from 0.87 to 0.90 (Table 7). They typically have low to moderate $\mathrm{Ti}$ concentrations, ranging from 0.23 to 0.68 wt $\% \mathrm{TiO}_{2}$, and relatively high $\mathrm{Cr}$ contents $\left(0.60-1.27 \mathrm{wt} \% \mathrm{Cr}_{2} \mathrm{O}_{3}\right) . \mathrm{Al}$ content may vary within individual samples and range from 2.23 to $3.91 \mathrm{wt} \% \mathrm{Al}_{2} \mathrm{O}_{3}$. Although shipboard observations suggested that the coarse-grained, diallage-textured pyroxenes could be metamorphic in origin, the $\mathrm{Al}$ and $\mathrm{Cr}$ contents indicate that primary magmatic chem- 
Table 7. Representative primary and secondary pyroxene compositions in Site 895 mafic rocks.

\begin{tabular}{|c|c|c|c|c|c|c|c|c|c|c|c|}
\hline \multirow{7}{*}{$\begin{array}{l}\text { Hole: } \\
\text { Core, section: } \\
\text { Interval }(\mathrm{cm}) \text { : } \\
\text { Piece: } \\
\text { Lithology: } \\
\text { Association: }\end{array}$} & \multicolumn{5}{|c|}{ Primary clinopyroxene } & \multicolumn{4}{|c|}{ Secondary clinopyroxene after olivine } & \multicolumn{2}{|c|}{ Secondary after clinopyroxene } \\
\hline & 895D & 895D & 895D & $895 \mathrm{E}$ & $895 \mathrm{E}$ & $895 \mathrm{D}$ & 895D & $895 \mathrm{E}$ & $895 \mathrm{E}$ & $895 \mathrm{D}$ & 895D \\
\hline & $8 \mathrm{R}-2$ & $8 \mathrm{R}-2$ & $8 \mathrm{R}-2$ & $6 \mathrm{R}-1$ & $6 \mathrm{R}-1$ & $7 R-1$ & $7 \mathrm{R}-1$ & IR-1 & IR-1 & $8 \mathrm{R}-2$ & $8 \mathrm{R}-2$ \\
\hline & $14-19$ & $14-19$ & $14-19$ & $15-17$ & $15-17$ & $81-84$ & $81-84$ & $83-87$ & $83-87$ & $14-19$ & $14-19$ \\
\hline & 2 & 2 & 2 & $4 \mathrm{~A}$ & $4 \mathrm{~A}$ & 13 & 13 & 9 & 9 & 2 & 2 \\
\hline & OLGB & OLGB & OLGB & GB & GB & TRO & TRO & OLGB & OLGB & OLGB & OLGB \\
\hline & & & & & & corona & corona & corona & snear zone & pun-apart & cpx nim \\
\hline $\mathrm{SiO}_{2}$ & 51.55 & 52.88 & 51.62 & 51.97 & 52.14 & 54.76 & 53.16 & 55.04 & 55.01 & 52.21 & 52.65 \\
\hline $\mathrm{TiO}_{2}$ & 0.31 & 0.30 & 0.44 & 0.38 & 0.30 & 0.02 & 0.01 & 0.00 & 0.02 & 0.11 & 0.03 \\
\hline $\mathrm{Al}_{2} \mathrm{O}_{3}$ & 3.91 & 2.82 & 3.11 & 2.78 & 3.44 & 0.69 & 1.54 & 0.71 & 0.08 & 0.45 & 0.23 \\
\hline $\mathrm{Cr}_{2} \mathrm{O}_{3}$ & 1.27 & 0.79 & 1.05 & 0.62 & 0.88 & 0.00 & 0.01 & 0.01 & 0.02 & 0.01 & 0.02 \\
\hline $\mathrm{Fe}_{2} \mathrm{O}_{3}$ & 0.00 & 0.00 & 0.00 & 0.00 & 0.00 & 0.00 & 0.00 & 0.00 & 0.00 & 0.00 & 0.00 \\
\hline $\mathrm{FeO}$ & 3.44 & 4.66 & 4.12 & 3.78 & 3.67 & 2.29 & 2.56 & 2.41 & 1.99 & 7.87 & 8.38 \\
\hline $\mathrm{MnO}$ & 0.14 & 0.15 & 0.12 & 0.13 & 0.13 & 0.10 & 0.08 & 0.08 & 0.10 & 0.55 & 0.67 \\
\hline $\mathrm{NiO}$ & 0.05 & 0.01 & 0.00 & 0.05 & 0.13 & 0.04 & 0.05 & 0.09 & 0.00 & 0.02 & 0.00 \\
\hline $\mathrm{MgO}$ & 16.52 & 17.18 & 16.54 & 17.00 & 16.87 & 18.65 & 19.41 & 19.26 & 18.09 & 13.38 & 12.76 \\
\hline $\mathrm{CaO}$ & 23.10 & 21.46 & 22.74 & 23.23 & 23.32 & 23.68 & 22.82 & 23.22 & 25.18 & 25.00 & 25.37 \\
\hline $\mathrm{Na}_{2} \mathrm{O}$ & 0.32 & 0.31 & 0.28 & 0.24 & 0.37 & 0.18 & 0.06 & 0.14 & 0.03 & 0.06 & 0.04 \\
\hline $\mathrm{K}_{2} \mathrm{O}$ & 0.00 & 0.02 & 0.02 & 0.01 & 0.00 & 0.00 & 0.00 & 0.04 & 0.01 & 0.00 & 0.00 \\
\hline $\mathrm{Cl}$ & 0.01 & 0.02 & 0.02 & 0.01 & 0.00 & 0.00 & 0.02 & 0.01 & 0.00 & 0.01 & 0.01 \\
\hline Total & 100.62 & 100.60 & 100.06 & 100.20 & 101.27 & 100.42 & 99.71 & 101.02 & 100.53 & 99.67 & 100.16 \\
\hline Si & 1.88 & 1.92 & 1.89 & 1.90 & 1.89 & 1.98 & 1.94 & 1.97 & 1.99 & 1.97 & 1.98 \\
\hline $\mathrm{Ti}$ & 0.01 & 0.01 & 0.01 & 0.01 & 0.01 & 0.00 & 0.00 & 0.00 & 0.00 & 0.00 & 0.00 \\
\hline $\mathrm{Al}$ & 0.17 & 0.12 & 0.13 & 0.12 & 0.15 & 0.03 & 0.07 & 0.03 & 0.00 & 0.02 & 0.01 \\
\hline $\mathrm{Cr}$ & 0.04 & 0.02 & 0.03 & 0.02 & 0.03 & 0.00 & 0.00 & 0.00 & 0.00 & 0.00 & 0.00 \\
\hline $\mathrm{Fe}^{3+}$ & 0.00 & 0.00 & 0.00 & 0.00 & 0.00 & 0.00 & 0.00 & 0.00 & 0.00 & 0.00 & 0.00 \\
\hline $\mathrm{Fe}^{2+}$ & 0.10 & 0.14 & 0.13 & 0.12 & 0.11 & 0.07 & 0.08 & 0.07 & 0.06 & 0.25 & 0.26 \\
\hline $\mathrm{Mn}$ & 0.00 & 0.00 & 0.00 & 0.00 & 0.00 & 0.00 & 0.00 & 0.00 & 0.00 & 0.02 & 0.02 \\
\hline $\mathrm{Ni}$ & 0.00 & 0.00 & 0.00 & 0.00 & 0.00 & 0.00 & 0.00 & 0.00 & 0.00 & 0.00 & 0.00 \\
\hline $\mathrm{Mg}$ & 0.90 & 0.93 & 0.90 & 0.93 & 0.91 & 1.00 & 1.05 & 1.03 & 0.98 & 0.75 & 0.72 \\
\hline $\mathrm{Ca}$ & 0.90 & 0.84 & 0.89 & 0.91 & 0.90 & 0.92 & 0.89 & 0.89 & 0.98 & 1.01 & 1.02 \\
\hline $\mathrm{Na}$ & 0.02 & 0.02 & 0.02 & 0.02 & 0.03 & 0.01 & 0.00 & 0.01 & 0.00 & 0.00 & 0.00 \\
\hline Sum cations & 4.02 & 4.01 & 4.02 & 4.03 & 4.03 & 4.01 & 4.03 & 4.02 & 4.01 & 4.02 & 4.02 \\
\hline $\mathrm{X}_{\mathrm{Mg}}$ & 0.90 & 0.87 & 0.88 & 0.89 & 0.89 & 0.94 & 0.93 & 0.93 & 0.94 & 0.75 & 0.73 \\
\hline $\mathrm{Al}^{\mathrm{IV}}$ & 0.12 & 0.08 & 0.11 & 0.10 & 0.11 & 0.02 & 0.06 & 0.03 & 0.00 & 0.02 & 0.01 \\
\hline $\mathrm{Al}^{\mathrm{VI}}$ & 0.05 & 0.04 & 0.03 & 0.02 & 0.04 & 0.01 & 0.00 & 0.00 & 0.00 & 0.00 & 0.00 \\
\hline Wollastonite & 0.45 & 0.42 & 0.44 & 0.45 & 0.45 & 0.46 & 0,43 & 0.44 & 0.48 & 0.50 & 0.51 \\
\hline Enstatite & 0.50 & 0.50 & 0.49 & 0.49 & 0.49 & 0.51 & 0.53 & 0.52 & 0.48 & 0.38 & 0.36 \\
\hline Ferrosilite & 0.06 & 0.08 & 0.07 & 0.06 & 0.06 & 0.03 & 0.04 & 0.04 & 0.03 & 0.12 & 0.13 \\
\hline
\end{tabular}

Notes: Formulas calculated on the basis of 12 oxygens and assuming $\mathrm{Fe}^{3+}=$ acmite, using a computer program provided by P. Ulmer (ETH-Zürich). Abbreviations as in Table 1. $\mathrm{X}_{\mathrm{Mg}}$ $=\mathrm{Mg} /(\mathrm{Mg}+\mathrm{Fe})$, with all $\mathrm{Fe}$ as $\mathrm{Fe}^{2+}$.

istries are preserved to a large extent. The clear secondary clinopyroxene that grow along the grain boundaries and as patches in primary grains, or in pull-apart structures, are more sialitic in composition (wollastonite components from $47 \%$ to $49 \%$ ) with distinctly lower $\mathrm{X}_{\mathrm{Mg}}(0.73-0.76)$ than the primary pyroxenes (Table 7).

The chemical compositions of the secondary clinopyroxene after olivine and in the shear zones are distinguished from primary clinopyroxene (Table 7 ) by distinctly low $\mathrm{Ti}$ and $\mathrm{Cr}$ contents (usually below or close to the detection limit) and by relatively constant high $\mathrm{X}_{\mathrm{Mg}}$, ranging from 0.93-0.95. In general, $\mathrm{Al}$ contents are also low, but are characteristically more variable, ranging from $0.08-1.54$ wt $\%$. Similar $\mathrm{Mg}$ to $\mathrm{Fe}$ ratios are observed in tremolite, with $\mathrm{X}_{\mathrm{Mg}}$ ranging from 0.89 to 0.95 . Although the amphiboles in the troctolitic and gabbroic rocks are characteristically tremolitic in composition, variations in $\mathrm{Al}$ content from 0.10 to $1.77 \mathrm{wt} \% \mathrm{Al}_{2} \mathrm{O}_{3}$ are observed within single clusters of tremolite pseudomorphs after olivine, with a tendency towards higher $\mathrm{Al}$ contents at the rims of the pseudomorphs (Table 8). Al-rich varieties, with 3.44 to 4.13 wt $\% \mathrm{Al}_{2} \mathrm{O}_{3}$, are locally associated with secondary clinopyroxene veinlets or occur in tremolite clusters as inclusions in primary clinopyroxene. An early generation of rare $\mathrm{Al}$-rich amphiboles, varying in composition from pargasitic hornblende to edenitic hornblende to hornblende, as well as local Fe-rich pargasite and pargasitic hornblende have been observed by Mével and Stamoudi (this volume).

Chlorite compositions are distinctly more variable than those of the other alteration phases. The compositions depend on bulk rock compositions, precursor mineralogies, type of occurrence (i.e., as replacement product or vein-filling phase), and the nature of the replacement (i.e., as coronitic replacement of olivine (ol) and plagio- clase, or as pseudomorphing phase after cpx or ol). The tri-octahedral variety, clinochlore, is the most common chlorite phase and is characterized by relatively high $\mathrm{Al}$ and $\mathrm{Mg}$ contents with variable total Fe. Tetrahedral occupation of $\mathrm{Al}$ in the 2:1 layer $\left(\mathrm{Al}^{\mathrm{IV}}\right)$ in general ranges from 0.89 to 1.29 . The higher $\mathrm{Al}^{\mathrm{IV}}$-concentrations correspond to (1) systematic increases in $\mathrm{Al}$ towards the rims of plagioclase grains in profiles measured across chlorite coronas; and (2) the alteration of plagioclase (Table 9; Fig. 6A). A Si-rich, Al-poorer variety of chlorite, penninite, occurs locally in pseudomorphs after olivine. The ratios of $\mathrm{Fe}$ to $\mathrm{Mg}\left(\mathrm{X}_{\mathrm{Fe}}=\mathrm{Fe}_{\text {(total) }} / \mathrm{Mg}+\mathrm{Fe}_{\text {(total) }}\right)$ of the different occurrences of chlorite reflect bulk rock chemistries and degrees of alteration (Laird, 1988). For example, $\mathrm{X}_{\mathrm{Fe}}$ in general ranges from 0.08 0.17 in troctolitic rocks and relatively fresh olivine gabbros, from $0.16-0.22$ in moderately to highly altered olivine gabbros, and $0.21-$ 0.31 in highly altered diallage gabbros. Chlorites that form pseudomorphs after primary clinopyroxene are characteristically $\mathrm{Cr}$-rich (1.30-3.54 wt \% $\mathrm{Cr}_{2} \mathrm{O}_{3}$ ) and commonly exhibit a decrease in $\mathrm{Cr}$ towards the rim of the pseudomorphed pyroxene grains (Table 9).

The variations in chlorite compositions are compared in Figure 6. Figure 6A illustrates the combined Tschermak substitutions $\left(\mathrm{Al}^{\mathrm{VI}} \mathrm{Al}^{\mathrm{VI}} \mathrm{Mg}_{-1} \mathrm{Si}_{-1}, \mathrm{CrAl}^{\mathrm{VI}} \mathrm{Mg}_{-1} \mathrm{Si}_{-1}\right.$, and $\mathrm{TiAl}^{\mathrm{VI}}{ }_{2} \mathrm{Mg}_{-1} \mathrm{Si}_{-2}$ ) with clinochlore-chamosite, $(\mathrm{Mg}, \mathrm{Fe})_{5} \mathrm{AlSi}_{3} \mathrm{AlO}_{10}(\mathrm{OH})_{8}$, as the origin. Most of the compositions plot above the 1:1 line from the origin (representing the Tschermak exchange in chlorite) and indicate dioctahedral substitution with octahedral vacancies (Laird, 1988). High octahedral vacancies are most pronounced in chlorites from veins and those associated with hydrogrossular as replacement products of plagioclase. The low Al-compositions, with values plotting at less than 0 , are typical of ultramafic rocks and rodingites and indicate that more than 
Table 8. Representative amphibole compositions from ultramafic and mafic lithologies at Site 895.

\begin{tabular}{|c|c|c|c|c|c|c|c|c|c|c|c|c|c|c|}
\hline $\begin{array}{l}\text { Hole: } \\
\text { Core, section: } \\
\text { Interval (cm): } \\
\text { Piece: } \\
\text { Lithology: } \\
\text { Association: }\end{array}$ & $\begin{array}{c}895 \mathrm{D} \\
2 \mathrm{R}-2 \\
29-32 \\
2 \\
\mathrm{HZ} \\
\text { local }\end{array}$ & $\begin{array}{c}895 \mathrm{D} \\
2 \mathrm{R}-2 \\
29-32 \\
2 \\
\mathrm{HZ} \\
\text { after cpx }\end{array}$ & $\begin{array}{c}895 \mathrm{D} \\
7 \mathrm{R}-1 \\
81-84 \\
13 \\
\text { TRO } \\
\text { ol psdm } \\
(+\mathrm{cpx})\end{array}$ & $\begin{array}{c}895 \mathrm{E} \\
\text { IR-1 } \\
83-87 \\
9 \\
\text { OLGB } \\
\text { ol psdm }\end{array}$ & $\begin{array}{c}895 \mathrm{E} \\
\text { IR-1 } \\
83-87 \\
9 \\
\text { OLGB } \\
\text { ol psdm }\end{array}$ & $\begin{array}{c}895 \mathrm{E} \\
2 \mathrm{R}-1 \\
9 \mathrm{I}-95 \\
14 \\
\text { OLGB } \\
\text { ol psdm }\end{array}$ & $\begin{array}{c}895 \mathrm{E} \\
2 \mathrm{R}-2 \\
6-10 \\
1 \\
\text { OLGB } \\
\text { ol psdm }\end{array}$ & $\begin{array}{c}895 E \\
2 R-2 \\
6-10 \\
1 \\
\text { OLGB } \\
\text { ol psdm } \\
\text { (rim) }\end{array}$ & $\begin{array}{c}895 \mathrm{E} \\
2 \mathrm{R}-2 \\
6-10 \\
1 \\
\text { OLGB } \\
\text { ol psdm } \\
(+ \text { cpx })\end{array}$ & $\begin{array}{c}895 \mathrm{E} \\
6 \mathrm{R}-1 \\
15-17 \\
4 \mathrm{~A} \\
\text { GB } \\
\text { ol psdm }\end{array}$ & $\begin{array}{c}895 \mathrm{E} \\
6 \mathrm{R}-1 \\
15-17 \\
4 \mathrm{~A} \\
\mathrm{~GB} \\
\text { ol psdm }\end{array}$ & $\begin{array}{c}895 \mathrm{E} \\
6 \mathrm{R}-1 \\
15-17 \\
4 \mathrm{~A} \\
\mathrm{~GB} \\
\text { ol psdm } \\
\text { (rim) }\end{array}$ & $\begin{array}{c}895 \mathrm{E} \\
6 \mathrm{R}-1 \\
15-17 \\
4 \mathrm{~A} \\
\mathrm{~GB} \\
\text { ol psdm } \\
\text { (rim) }\end{array}$ & $\begin{array}{c}895 \mathrm{E} \\
6 \mathrm{R}-1 \\
15-17 \\
4 \mathrm{~A} \\
\mathrm{~GB} \\
\text { in cpx }\end{array}$ \\
\hline $\begin{array}{l}\mathrm{SiO}_{2} \\
\mathrm{TiO}_{2} \\
\mathrm{Al}_{2} \mathrm{O} \\
\mathrm{Cr}_{2} \mathrm{O} \\
\mathrm{FeO}^{*} \\
\mathrm{MnO} \\
\mathrm{NiO} \\
\mathrm{MgO} \\
\mathrm{CaO} \\
\mathrm{Na}_{2} \mathrm{O} \\
\mathrm{K}_{2} \mathrm{O} \\
\mathrm{Cl} \\
\mathrm{H}_{2} \mathrm{O} \\
\text { Total }\end{array}$ & $\begin{array}{r}57.55 \\
0.01 \\
30.09 \\
30.17 \\
2.37 \\
0.09 \\
0.03 \\
22.94 \\
13.41 \\
0.06 \\
0.03 \\
0.03 \\
2.16 \\
98.92\end{array}$ & $\begin{array}{r}55.33 \\
0.00 \\
2.58 \\
0.74 \\
2.43 \\
0.08 \\
0.09 \\
22.69 \\
12.28 \\
0.74 \\
0.03 \\
0.04 \\
2.15 \\
99.18\end{array}$ & $\begin{array}{r}53.19 \\
0.04 \\
3.06 \\
0.07 \\
3.45 \\
0.05 \\
0.12 \\
24.52 \\
13.60 \\
0.25 \\
0.01 \\
0.01 \\
2.17 \\
100.54\end{array}$ & $\begin{array}{r}58.84 \\
0.00 \\
0.12 \\
0.00 \\
2.00 \\
0.03 \\
0.11 \\
23.77 \\
13.91 \\
0.06 \\
0.02 \\
0.00 \\
2.22 \\
101.08\end{array}$ & $\begin{array}{r}57.59 \\
0.03 \\
0.55 \\
0.04 \\
2.45 \\
0.09 \\
0.10 \\
22.93 \\
13.65 \\
0.19 \\
0.03 \\
0.01 \\
2.18 \\
99.83\end{array}$ & $\begin{array}{r}57.56 \\
0.03 \\
0.30 \\
0.01 \\
3.16 \\
0.06 \\
0.21 \\
22.74 \\
13.17 \\
0.09 \\
0.00 \\
0.01 \\
2.17 \\
99.50\end{array}$ & $\begin{array}{r}58.12 \\
0.01 \\
0.54 \\
0.02 \\
2.96 \\
0.03 \\
0.12 \\
22.62 \\
13.63 \\
0.16 \\
0.00 \\
0.00 \\
2.19 \\
100.41\end{array}$ & $\begin{array}{r}56.79 \\
0.03 \\
1.32 \\
0.00 \\
3.30 \\
0.06 \\
0.15 \\
22.75 \\
13.62 \\
0.44 \\
0.02 \\
0.00 \\
2.19 \\
100.66\end{array}$ & $\begin{array}{r}53.91 \\
0.04 \\
3.95 \\
0.04 \\
4.66 \\
0.09 \\
0.14 \\
23.23 \\
11.58 \\
0.21 \\
0.01 \\
0.02 \\
2.16 \\
100.06\end{array}$ & $\begin{array}{r}58.36 \\
0.05 \\
0.16 \\
0.04 \\
2.26 \\
0.04 \\
0.16 \\
23.78 \\
13.66 \\
0.07 \\
0.02 \\
0.02 \\
2.20 \\
100.81\end{array}$ & $\begin{array}{r}57.26 \\
0.02 \\
0.27 \\
0.00 \\
3.85 \\
0.09 \\
0.20 \\
22.77 \\
13.53 \\
0.11 \\
0.04 \\
0.01 \\
2.18 \\
100.32\end{array}$ & $\begin{array}{r}56.43 \\
0.03 \\
0.82 \\
0.04 \\
3.91 \\
0.05 \\
0.11 \\
22.55 \\
12.85 \\
0.22 \\
0.04 \\
0.05 \\
2.14 \\
99.25\end{array}$ & $\begin{array}{r}55.99 \\
0.03 \\
1.67 \\
0.01 \\
4.09 \\
0.10 \\
0.15 \\
22.76 \\
13.03 \\
0.32 \\
0.05 \\
0.01 \\
2.17 \\
100.37\end{array}$ & $\begin{array}{r}53.54 \\
0.05 \\
3.44 \\
0.04 \\
4.38 \\
0.06 \\
0.12 \\
22.96 \\
11.82 \\
0.22 \\
0.01 \\
0.02 \\
2.14 \\
98.78\end{array}$ \\
\hline $\begin{array}{l}\mathrm{Si} \\
\mathrm{Ti} \\
\mathrm{Al} \\
\mathrm{Cr} \\
\mathrm{Fe}^{2+} \\
\mathrm{Mn} \\
\mathrm{Ni} \\
\mathrm{Mg} \\
\mathrm{Ca} \\
\mathrm{Na} \\
\mathrm{K} \\
\mathrm{Cl} \\
\mathrm{H} \\
\text { Sum cations }\end{array}$ & $\begin{array}{l}7.96 \\
0.00 \\
0.01 \\
0.02 \\
0.27 \\
0.01 \\
0.00 \\
4.73 \\
1.99 \\
0.02 \\
0.00 \\
0.01 \\
1.99 \\
15.03\end{array}$ & $\begin{array}{l}7.67 \\
0.00 \\
0.42 \\
0.08 \\
0.28 \\
0.01 \\
0.01 \\
4.69 \\
1.82 \\
0.20 \\
0.01 \\
0.01 \\
1.99 \\
15.19\end{array}$ & $\begin{array}{l}7.35 \\
0.00 \\
0.50 \\
0.01 \\
0.40 \\
0.01 \\
0.01 \\
5.05 \\
2.02 \\
0.07 \\
0.00 \\
0.00 \\
2.00 \\
15.42\end{array}$ & $\begin{array}{l}7.96 \\
0.00 \\
0.02 \\
0.00 \\
0.23 \\
0.00 \\
0.01 \\
4.79 \\
2.01 \\
0.01 \\
0.00 \\
0.00 \\
2.00 \\
15.04\end{array}$ & $\begin{array}{l}7.91 \\
0.00 \\
0.09 \\
0.00 \\
0.28 \\
0.01 \\
0.01 \\
4.70 \\
2.01 \\
0.05 \\
0.00 \\
0.00 \\
2.00 \\
15.07\end{array}$ & $\begin{array}{l}7.94 \\
0.00 \\
0.05 \\
0.00 \\
0.36 \\
0.01 \\
0.02 \\
4.68 \\
1.95 \\
0.02 \\
0.00 \\
0.00 \\
2.00 \\
15.04\end{array}$ & $\begin{array}{l}7.94 \\
0.00 \\
0.09 \\
0.00 \\
0.34 \\
0.00 \\
0.01 \\
4.61 \\
2.00 \\
0.04 \\
0.00 \\
0.00 \\
2.00 \\
15.04\end{array}$ & $\begin{array}{l}7.78 \\
0.00 \\
0.21 \\
0.00 \\
0.38 \\
0.01 \\
0.02 \\
4.65 \\
2.00 \\
0.12 \\
0.00 \\
0.00 \\
2.00 \\
15.17\end{array}$ & $\begin{array}{l}7.46 \\
0.00 \\
0.64 \\
0.00 \\
0.54 \\
0.01 \\
0.02 \\
4.79 \\
1.72 \\
0.06 \\
0.00 \\
0.00 \\
2.00 \\
15.24\end{array}$ & $\begin{array}{l}7.93 \\
0.00 \\
0.03 \\
0.00 \\
0.26 \\
0.00 \\
0.02 \\
4.81 \\
1.99 \\
0.02 \\
0.00 \\
0.00 \\
2.00 \\
15.06\end{array}$ & $\begin{array}{l}7.88 \\
0.00 \\
0.04 \\
0.00 \\
0.44 \\
0.01 \\
0.02 \\
4.67 \\
2.00 \\
0.03 \\
0.01 \\
0.00 \\
2.00 \\
15.11\end{array}$ & $\begin{array}{l}7.85 \\
0.00 \\
0.13 \\
0.00 \\
0.46 \\
0.01 \\
0.01 \\
4.67 \\
1.91 \\
0.06 \\
0.01 \\
0.01 \\
1.99 \\
15.12\end{array}$ & $\begin{array}{l}7.72 \\
0.00 \\
0.27 \\
0.00 \\
0.47 \\
0.01 \\
0.02 \\
4.68 \\
1.93 \\
0.09 \\
0.01 \\
0.00 \\
2.00 \\
15.19\end{array}$ & $\begin{array}{r}7.50 \\
0.00 \\
0.57 \\
0.00 \\
0.51 \\
0.01 \\
0.01 \\
4.79 \\
1.77 \\
0.06 \\
0.00 \\
0.00 \\
2.00 \\
15.24\end{array}$ \\
\hline $\begin{array}{l}\mathrm{X}_{\mathrm{Mg}_{\mathrm{g}}(\mathrm{Fe} \text { tol) }} \\
\mathrm{Al}^{\mathrm{IV}} \\
\mathrm{Al}^{\mathrm{VI}} \\
\mathrm{Na}^{\mathrm{A}}\end{array}$ & $\begin{array}{l}0.94 \\
0.01 \\
0.00 \\
0.01\end{array}$ & $\begin{array}{l}0.94 \\
0.32 \\
0.08 \\
0.18\end{array}$ & $\begin{array}{l}0.93 \\
0.50 \\
0.00 \\
0.07\end{array}$ & $\begin{array}{l}0.96 \\
0.02 \\
0.00 \\
0.01\end{array}$ & $\begin{array}{l}0.94 \\
0.09 \\
0.00 \\
0.05\end{array}$ & $\begin{array}{l}0.93 \\
0.05 \\
0.00 \\
0.03\end{array}$ & $\begin{array}{l}0.93 \\
0.06 \\
0.03 \\
0.04\end{array}$ & $\begin{array}{l}0.93 \\
0.21 \\
0.00 \\
0.12\end{array}$ & $\begin{array}{l}0.90 \\
0.54 \\
0.10 \\
0.06\end{array}$ & $\begin{array}{l}0.95 \\
0.03 \\
0.00 \\
0.02\end{array}$ & $\begin{array}{l}0.91 \\
0.04 \\
0.00 \\
0.03\end{array}$ & $\begin{array}{l}0.91 \\
0.14 \\
0.00 \\
0.06\end{array}$ & $\begin{array}{l}0.91 \\
0.27 \\
0.00 \\
0.09\end{array}$ & $\begin{array}{l}0.90 \\
0.50 \\
0.07 \\
0.06\end{array}$ \\
\hline
\end{tabular}

Notes: Cation proportions calculated on the basis of 23 oxygens and fixed total $\mathrm{Fe}$ as $\mathrm{Fe}^{2+}$ using a computer program provided by $\mathrm{P} . \mathrm{Ulmer}(\mathrm{ETH}-\mathrm{Zürich}) . \mathrm{X}$ Abbreviations: local = local abundance in $\mathrm{HZ}$; ol psdm = pseudomorphic replacement of olivine; ol psdm $(+\mathrm{cpx})=$ pseudomorphic replacement of olivine together with secondary clinopyroxene; ol psdm $(\mathrm{rim})=$ rim of olivine pseudomorph at contact to chlorite corona; in $\mathrm{cpx}=$ in primary clinopyroxene. Other abbreviations as in Table 1 .

three-fourths of the tetrahedral sites are filled by Si (Laird, 1988). Nearly linear variations in the tetrahedral substitution as a function of $\mathrm{X}_{\mathrm{Mg}}$ are seen in Figure $6 \mathrm{~B}$ and clearly show the relatively high $\mathrm{Mg}$ contents of the Site 895 chlorites and the relationship to bulk composition. Although linear correlations are uncommon in metabasic rocks (Laird, 1988), a similar relationship in chlorite, with an increase in $\mathrm{Al}$ associated with a decrease in $\mathrm{Si}$, in low pressure greenschist and epidote amphibolite facies metabasalts, has been reported by Maruyama et al. (1983).

Representative chemical compositions of the calc-silicate phases after plagioclase are given in Table 10. Prehnite exhibits very constant compositions throughout the samples analyzed, with a nearly stoichiometric 3:1 ratio of $\mathrm{Si}$ to $\mathrm{Al}^{\mathrm{IV}}$ and no distinction between veinfilling prehnite and prehnite as alteration of plagioclase. The compositions are characteristically $\mathrm{Fe}$-poor with $\mathrm{Fe} /(\mathrm{Fe}+\mathrm{Al})$ ratios generally much less than 0.1. Analysis of the red-brown to clear, microcrystalline, nearly isotropic phases, as alteration products of plagioclase and vein-filling material and interpreted to be hydrogrossular-type minerals, proved extremely difficult. Electron microprobe results show that the compositions of these phases are transitional between prehnite and the theoretical hydrogrossular end-member $\mathrm{Ca}_{3} \mathrm{Al}_{2}\left(\mathrm{SiO}_{4}\right)_{3-\mathrm{m}}$ $(\mathrm{OH})_{4 \mathrm{~m}}$, and in some cases may even be mixtures of Ca-Na zeolite and prehnite. Ratios of $\mathrm{Si}$ to $\mathrm{Al}^{\mathrm{IV}}$ ratios vary from 1.11 to 1.73. Large variations in the total weight percent of the elements analyzed suggest that the total amount of water is highly variable (Table 10). The presence of thomsonite, a Ca-Na zeolite of the natrolite group, in altered plagioclase and in veins has been determined by electron microprobe analysis and was confirmed by XRD determinations. Although slight variations in $\mathrm{Na}$ and $\mathrm{Ca}$ concentrations were observed between the various rock types, the compositions within an individual sample remain constant, with nearly stoichiometric $1: 1$ ratios of $\mathrm{Si}$ to $\mathrm{Al}$, and no distinction between vein- and replacement-zeolite compositions (Table 10).

\section{STABLE ISOTOPE CONSTRAINTS ON HYDROTHERMAL ALTERATION}

Gabbroic cumulates and mantle peridotites that have not interacted with external fluids should have bulk rock oxygen isotope ratios within the range known for fresh mid-ocean ridge basalts $(=5.8 \pm$ $0.3 \%$; e.g., Taylor, 1968; references in Muehlenbachs, 1987; Kyser, 1987). Seawater-rock interactions produce shifts away from these reference values, whereby the final composition of the rock will reflect the temperatures of alteration and fluid/rock ratios (e.g., Taylor, 1977, 1984; Ito and Clayton, 1983). The oxygen, hydrogen, and carbon isotope results of fresh and altered phases as well as vein minerals from Holes $895 \mathrm{C}$ through $895 \mathrm{E}$ are given in Table 11 and are shown graphically in Figures 7 through 11. Stable isotope data of the Hess Deep lower crust and shallow mantle, combined with petrology and phase chemistry, provide new constraints from a fast-spreading environment on the general models of hydrothermal alteration and oxygen isotope exchange in the oceanic crust (compare, e.g., Gregory and Taylor, 1981; Muehlenbachs, 1987; Agrinier et al., 1988; Lécuyer and Fourcade, 1991; Stakes and Taylor, 1992). These data allow an assessment of the degree to which seawater penetrates and reacts 
Table 9. Representative chlorite compositions in ultramafic and mafic lithologies at Site 895.

\begin{tabular}{|c|c|c|c|c|c|c|c|c|c|c|c|c|}
\hline $\begin{array}{l}\text { Hole: } \\
\text { Core, section: } \\
\text { Interval (cm): } \\
\text { Piece: } \\
\text { Lithology: } \\
\text { Association: }\end{array}$ & $\begin{array}{l}895 \mathrm{C} \\
4 \mathrm{R}-2 \\
52-54 \\
\text { ID } \\
\text { IDU } \\
\text { vein }\end{array}$ & $\begin{array}{l}895 \mathrm{C} \\
4 \mathrm{R}-2 \\
52-54 \\
\text { ID } \\
\text { IDU } \\
\text { local }\end{array}$ & $\begin{array}{c}895 \mathrm{C} \\
4 \mathrm{R}-3 \\
13-16 \\
2 \mathrm{~A} \\
\text { IDU } \\
\text { plg psdm }\end{array}$ & $\begin{array}{l}895 \mathrm{C} \\
4 \mathrm{R}-3 \\
13-16 \\
2 \mathrm{~A} \\
\mathrm{IDU} \\
\text { vein }\end{array}$ & $\begin{array}{c}895 \mathrm{C} \\
4 \mathrm{R}-3 \\
13-16 \\
2 \mathrm{~A} \\
\text { IDU } \\
\text { plg psdm }\end{array}$ & $\begin{array}{c}895 \mathrm{C} \\
4 \mathrm{R}-3 \\
13-16 \\
2 \mathrm{~A} \\
\text { IDU } \\
\text { plg psdm }\end{array}$ & $\begin{array}{c}895 \mathrm{D} \\
7 \mathrm{R}-1 \\
81-84 \\
13 \\
\text { TRO } \\
\text { corona } \\
\text { center }\end{array}$ & $\begin{array}{c}895 \mathrm{D} \\
7 \mathrm{R}-1 \\
81-84 \\
13 \\
\text { TRO } \\
\text { corona } \\
\text { (rim/plg) }\end{array}$ & $\begin{array}{c}895 \mathrm{D} \\
7 \mathrm{R}-1 \\
81-84 \\
13 \\
\text { TRO } \\
\text { ol psdm }\end{array}$ & $\begin{array}{c}895 \mathrm{D} \\
7 \mathrm{R}-1 \\
81-84 \\
13 \\
\text { TRO } \\
\text { ol psdm } \\
\text { (rim) }\end{array}$ & $\begin{array}{c}895 \mathrm{D} \\
7 \mathrm{R}-1 \\
81-84 \\
13 \\
\text { TRO } \\
\text { vein }\end{array}$ & $\begin{array}{c}895 \mathrm{D} \\
8 \mathrm{R}-2 \\
14-19 \\
2 \\
\text { OLGB } \\
\text { in pre } \\
\text { cluster }\end{array}$ \\
\hline $\begin{array}{l}\mathrm{SiO}_{2} \\
\mathrm{TiO}_{2} \\
\mathrm{Al}_{2} \mathrm{O}_{3} \\
\mathrm{Cr}_{2} \mathrm{O}_{3} \\
\mathrm{Fe}_{2} \mathrm{O}_{3} \\
\mathrm{FeO} \\
\mathrm{MnO} \\
\mathrm{MgO} \\
\mathrm{NiO} \\
\mathrm{CaO} \\
\mathrm{Na}_{2} \mathrm{O} \\
\mathrm{K}_{2} \mathrm{O} \\
\mathrm{Cl} \\
\mathrm{H}_{2} \mathrm{O} \\
\text { Total }\end{array}$ & $\begin{array}{r}29.16 \\
0.02 \\
20.64 \\
0.00 \\
0.00 \\
8.35 \\
0.16 \\
28.26 \\
0.11 \\
0.11 \\
0.04 \\
0.04 \\
0.01 \\
12.35 \\
99.25\end{array}$ & $\begin{array}{r}28.71 \\
0.01 \\
20.79 \\
0.05 \\
0.03 \\
9.76 \\
0.17 \\
27.36 \\
0.14 \\
0.04 \\
0.06 \\
0.08 \\
0.04 \\
12.28 \\
99.51\end{array}$ & $\begin{array}{r}33.64 \\
0.02 \\
14.27 \\
0.01 \\
0.00 \\
5.26 \\
0.09 \\
32.90 \\
0.19 \\
0.02 \\
0.03 \\
0.09 \\
0.06 \\
12.51 \\
99.08\end{array}$ & $\begin{array}{r}31.84 \\
0.03 \\
17.99 \\
0.00 \\
0.00 \\
4.87 \\
0.09 \\
31.83 \\
0.15 \\
0.02 \\
0.05 \\
0.08 \\
0.03 \\
12.61 \\
99.58\end{array}$ & $\begin{array}{r}27.40 \\
0.00 \\
21.91 \\
0.00 \\
0.00 \\
13.07 \\
0.17 \\
24.30 \\
0.02 \\
0.06 \\
0.06 \\
0.10 \\
0.03 \\
12.06 \\
99.20\end{array}$ & $\begin{array}{r}31.68 \\
0.01 \\
18.32 \\
0.04 \\
0.00 \\
5.14 \\
0.11 \\
31.64 \\
0.12 \\
0.05 \\
0.05 \\
0.08 \\
0.04 \\
12.62 \\
99.89\end{array}$ & $\begin{array}{r}31.38 \\
0.02 \\
19.41 \\
0.03 \\
0.00 \\
6.17 \\
0.06 \\
30.45 \\
0.20 \\
0.09 \\
0.21 \\
0.08 \\
0.09 \\
12.65 \\
100.83\end{array}$ & $\begin{array}{r}29.48 \\
0.01 \\
21.05 \\
0.00 \\
0.00 \\
8.20 \\
0.20 \\
28.26 \\
0.03 \\
0.10 \\
0.14 \\
0.04 \\
0.00 \\
12.45 \\
99.97\end{array}$ & $\begin{array}{r}26.53 \\
0.01 \\
24.47 \\
0.01 \\
0.00 \\
15.52 \\
0.54 \\
20.59 \\
0.00 \\
0.14 \\
0.08 \\
0.01 \\
0.00 \\
12.03 \\
99.93\end{array}$ & $\begin{array}{r}33.70 \\
0.02 \\
14.60 \\
0.02 \\
0.00 \\
5.70 \\
0.04 \\
33.05 \\
0.20 \\
0.04 \\
0.05 \\
0.01 \\
0.01 \\
12.63 \\
100.07\end{array}$ & $\begin{array}{r}34.49 \\
0.00 \\
15.16 \\
0.00 \\
0.00 \\
4.88 \\
0.24 \\
32.72 \\
0.00 \\
0.16 \\
0.03 \\
0.02 \\
0.02 \\
12.74 \\
100.45\end{array}$ & $\begin{array}{r}28.07 \\
0.02 \\
21.12 \\
0.05 \\
0.00 \\
11.76 \\
0.16 \\
25.50 \\
0.27 \\
0.05 \\
0.07 \\
0.02 \\
0.02 \\
12.14 \\
99.24\end{array}$ \\
\hline & \multicolumn{12}{|c|}{ Tetrahedral cations in 2:1 layer } \\
\hline $\begin{array}{l}\mathrm{Si} \\
\mathrm{Al}^{\mathrm{IV}}\end{array}$ & $\begin{array}{l}2.83 \\
1.17\end{array}$ & $\begin{array}{l}2.80 \\
1.20\end{array}$ & $\begin{array}{l}3.22 \\
0.78\end{array}$ & $\begin{array}{l}3.03 \\
0.97\end{array}$ & $\begin{array}{l}2.72 \\
1.28\end{array}$ & $\begin{array}{l}3.01 \\
0.99\end{array}$ & $\begin{array}{l}2.97 \\
1.03\end{array}$ & $\begin{array}{l}2.84 \\
1.16\end{array}$ & $\begin{array}{l}2.64 \\
1.36\end{array}$ & $\begin{array}{l}3.20 \\
0.80\end{array}$ & $\begin{array}{l}3.24 \\
0.76\end{array}$ & $\begin{array}{l}2.77 \\
1.23\end{array}$ \\
\hline $\begin{array}{c}\mathrm{Mg} \\
\mathrm{Al}^{\mathrm{Vi}} \\
\mathrm{Fe}^{3+} \\
\mathrm{Fe}^{2+} \\
\mathrm{Mn} \\
\mathrm{Ni} \\
\text { Total }\end{array}$ & $\begin{array}{l}2.09 \\
0.20 \\
0.00 \\
0.68 \\
0.01 \\
0.01 \\
2.99\end{array}$ & $\begin{array}{l}1.98 \\
0.20 \\
0.00 \\
0.80 \\
0.01 \\
0.01 \\
3.00\end{array}$ & $\begin{array}{l}2.53 \\
0.00 \\
0.00 \\
0.42 \\
0.01 \\
0.01 \\
2.97\end{array}$ & $\begin{array}{l}2.48 \\
0.07 \\
0.00 \\
0.39 \\
0.01 \\
0.01 \\
2.96\end{array}$ & $\begin{array}{l}\text { Oct } \\
1.60 \\
0.29 \\
0.00 \\
1.09 \\
0.01 \\
0.00 \\
2.99\end{array}$ & $\begin{array}{c}\text { rahedral cat } \\
2.47 \\
0.07 \\
0.00 \\
0.41 \\
0.01 \\
0.01 \\
2.97\end{array}$ & $\begin{array}{c}\text { ns in } 2: 1 \\
2.30 \\
0.14 \\
0.00 \\
0.49 \\
0.00 \\
0.02 \\
2.94\end{array}$ & $\begin{array}{l}2.06 \\
0.23 \\
0.00 \\
0.66 \\
0.02 \\
0.00 \\
2.97\end{array}$ & $\begin{array}{l}1.06 \\
0.52 \\
0.00 \\
1.29 \\
0.05 \\
0.00 \\
2.92\end{array}$ & $\begin{array}{l}2.51 \\
0.00 \\
0.00 \\
0.45 \\
0.00 \\
0.01 \\
2.98\end{array}$ & $\begin{array}{l}2.51 \\
0.00 \\
0.00 \\
0.38 \\
0.02 \\
0.00 \\
2.92\end{array}$ & $\begin{array}{l}1.75 \\
0.24 \\
0.00 \\
0.97 \\
0.01 \\
0.02 \\
3.00\end{array}$ \\
\hline $\begin{array}{c}\mathrm{Mg} \\
\mathrm{Al}^{\mathrm{V}} \\
\mathrm{Cr} \\
\mathrm{Ti} \\
\text { Total }\end{array}$ & $\begin{array}{l}2.00 \\
1.00 \\
0.00 \\
0.00 \\
3.00\end{array}$ & $\begin{array}{l}2.00 \\
0.99 \\
0.00 \\
0.00 \\
3.00\end{array}$ & $\begin{array}{l}2.17 \\
0.83 \\
0.00 \\
0.00 \\
3.00\end{array}$ & $\begin{array}{l}2.03 \\
0.97 \\
0.00 \\
0.00 \\
3.00\end{array}$ & $\begin{array}{c}\text { Octrahedral } \\
2.00 \\
1.00 \\
0.00 \\
0.00 \\
3.00\end{array}$ & $\begin{array}{c}\text { cations in } \\
2.01 \\
0.99 \\
0.00 \\
0.00 \\
3.00\end{array}$ & $\begin{array}{c}\text { terlayer }( \\
2.00 \\
1.00 \\
0.00 \\
0.00 \\
3.00\end{array}$ & $\begin{array}{c}\text { ucite layer) } \\
2.00 \\
1.00 \\
0.00 \\
0.00 \\
3.00\end{array}$ & $\begin{array}{l}2.00 \\
1.00 \\
0.00 \\
0.00 \\
3.00\end{array}$ & $\begin{array}{l}2.16 \\
0.83 \\
0.00 \\
0.00 \\
3.00\end{array}$ & $\begin{array}{l}2.08 \\
0.92 \\
0.00 \\
0.00 \\
3.00\end{array}$ & $\begin{array}{l}2.00 \\
0.99 \\
0.00 \\
0.00 \\
3.00\end{array}$ \\
\hline $\begin{array}{l}\mathrm{Ca} \\
\mathrm{Na} \\
\mathrm{K}\end{array}$ & $\begin{array}{l}0.01 \\
0.01 \\
0.01\end{array}$ & $\begin{array}{l}0.00 \\
0.01 \\
0.01\end{array}$ & $\begin{array}{l}0.00 \\
0.01 \\
0.01\end{array}$ & $\begin{array}{l}0.00 \\
0.01 \\
0.01\end{array}$ & $\begin{array}{l}0.01 \\
0.01 \\
0.01\end{array}$ & $\begin{array}{l}0.01 \\
0.01 \\
0.01\end{array}$ & $\begin{array}{l}0.01 \\
0.01 \\
0.01\end{array}$ & $\begin{array}{l}0.01 \\
0.04 \\
0.01\end{array}$ & $\begin{array}{l}0.01 \\
0.03 \\
0.01\end{array}$ & $\begin{array}{l}0.02 \\
0.01 \\
0.00\end{array}$ & $\begin{array}{l}0.00 \\
0.01 \\
0.00\end{array}$ & $\begin{array}{l}0.02 \\
0.01 \\
0.00\end{array}$ \\
\hline & \multicolumn{12}{|c|}{ Exchange vectors } \\
\hline $\begin{array}{l}\text { xAITS } \\
\text { xTD } \\
\text { xCrTS } \\
\text { Xmg }\end{array}$ & $\begin{array}{l}0.17 \\
0.03 \\
0.00 \\
0.86\end{array}$ & $\begin{array}{l}0.19 \\
0.03 \\
0.00 \\
0.83\end{array}$ & $\begin{array}{r}-0.22 \\
0.03 \\
0.00 \\
0.92\end{array}$ & $\begin{array}{r}-0.03 \\
0.04 \\
0.00 \\
0.92\end{array}$ & $\begin{array}{l}0.27 \\
0.01 \\
0.00 \\
0.77\end{array}$ & $\begin{array}{r}-0.01 \\
0.04 \\
0.00 \\
0.92\end{array}$ & $\begin{array}{l}0.02 \\
0.06 \\
0.00 \\
0.90\end{array}$ & $\begin{array}{l}0.16 \\
0.03 \\
0.00 \\
0.86\end{array}$ & $\begin{array}{l}0.35 \\
0.08 \\
0.00 \\
0.70\end{array}$ & $\begin{array}{r}-0.20 \\
0.02 \\
0.00 \\
0.91\end{array}$ & $\begin{array}{r}-0.24 \\
0.08 \\
0.00 \\
0.92\end{array}$ & $\begin{array}{l}0.22 \\
0.00 \\
0.00 \\
0.79\end{array}$ \\
\hline
\end{tabular}

Notes: Fomulas are calculated using a computer program provided by $\mathrm{D}$. Sidler. Exchange vectors are defined as: $\mathrm{Al}-\mathrm{Tschermak}(\mathrm{xALTS})=\mathrm{Al}_{2} \mathrm{Mg}_{1} \mathrm{Si}_{1} ; \mathrm{Cr}-\mathrm{Tschermak}(\mathrm{xCRTS})=$ $\mathrm{CrAlMg} \mathrm{Si}_{-1} ; \mathrm{Tri}-$ to dioctahedral $(\mathrm{xTD})=\mathrm{Al}_{2} \mathrm{Mg}_{-3} ; \mathrm{xMg}=\mathrm{Mg} /\left(\mathrm{Mg}+\mathrm{Fe}_{\text {total }}\right)$. Association abbreviations are: local = local occurrence in harzburgite; ol psdm $=$ pseudomorphic replacement of olivine; ol psdm (rim) = rim of olivine at contact to chlorite corona; ol psdm $(+$ tre $)=$ pseudomorphic replacement of olivine together with tremolite; cpx psdm= primary clinopyroxene; plg pseud $=$ static replacement of plagioclase; rim/plg = composition of chlorite corona rimming plagioclase; pre $=$ prehnite .

with the lower oceanic crust and shallow mantle, and provide constraints on the role of magmatic volatiles and metamorphic fluids in deep-seated hydrothermal systems.

\section{Ultramafic Rocks}

Oxygen and hydrogen isotope data have been used in the past as tracers of fluid-rock interaction during serpentinization of ultramafic rocks from both ophiolites and oceanic sections. In spite of uncertainties in the serpentine-water fractionation curves (in particular for hydrogen; see discussions in Wenner and Taylor, 1971, 1973, 1974; Sakai and Tsutsumi, 1978; Kyser, 1987; Sakai et al., 1990), the oxygen and hydrogen isotope signatures of the Hess Deep peridotites, as well as thermal decomposition experiments carried out on two pervasively serpentinized peridotites, provide important information on the origin of the serpentinizing fluids and the extent of seawater penetration and fluid-rock interaction in the shallow mantle. The combination of stable isotope geochemistry and petrologic data may further constrain the role of serpentinization in the exhumation of the lower crustal-shallow mantle sequence and allow the two models proposed by Francheteau et al. (1990) for the tectonic evolution of the Hess Deep to be evaluated.

\section{Stable Isotope Ratios of Serpentine}

More than 60 oxygen and hydrogen isotope measurements were systematically made on rock-serpentine and vein-serpentine separate samples from Hole $895 \mathrm{C}$ to Hole $895 \mathrm{E}$. Vein serpentine was separated from serpentine + magnetite \pm chlorite veins of Type 2 , which are generally thicker than other vein types, allowing sufficient material recovery. The purity of the rock serpentine separates is in most cases $>95 \%$, whereby in the pervasively altered dunite samples the measured isotopic value can approximately be considered a whole rock value.

The $\delta^{18} \mathrm{O}$ values of rock-serpentine separates are characteristically low, ranging from $3.2 \%$ to $5.4 \%$ (Figs. 7 through 9; Table 11). They are very similar to previously measured values in Hess Deep ultramafic rocks (Agrinier et al., in press), and are consistent with a large number of oceanic serpentinites examined to date (Wenner and Taylor, 1973; Sheppard, 1980; Sakai et al., 1990). No significant correlation between the oxygen isotope ratios and rock type (dunite/ 
Table 9 (continued).

\begin{tabular}{|c|c|c|c|c|c|c|c|c|c|c|c|c|c|}
\hline $\begin{array}{c}895 \mathrm{D} \\
8 \mathrm{R}-2 \\
14-19 \\
2 \\
\text { OLGB } \\
\text { cpx psdm } \\
\text { (rim) }\end{array}$ & $\begin{array}{c}895 \mathrm{D} \\
8 \mathrm{R}-2 \\
14-19 \\
2 \\
\text { OLGB } \\
\text { cpx psdm } \\
\text { (center) }\end{array}$ & $\begin{array}{c}895 \mathrm{E} \\
1 \mathrm{R}-1 \\
83-87 \\
9 \\
\text { OLGB } \\
\text { corona } \\
\text { (center) }\end{array}$ & $\begin{array}{c}895 \mathrm{E} \\
1 \mathrm{R}-1 \\
83-87 \\
9 \\
\text { OLGB } \\
\text { corona } \\
\text { (rim/plg) }\end{array}$ & $\begin{array}{c}895 \mathrm{E} \\
1 \mathrm{R}-1 \\
83-87 \\
9 \\
\text { OLGB } \\
\text { ol psdm } \\
\text { (green) }\end{array}$ & $\begin{array}{c}895 \mathrm{E} \\
2 \mathrm{R}-1 \\
91-95 \\
14 \\
\text { OLGB } \\
\text { sheared } \\
\text { (corona) }\end{array}$ & $\begin{array}{c}895 \mathrm{E} \\
2 \mathrm{R}-1 \\
91-95 \\
14 \\
\text { OLGB } \\
\text { sheared } \\
\text { (corona rim) }\end{array}$ & $\begin{array}{c}895 \mathrm{E} \\
2 \mathrm{R}-1 \\
91-95 \\
14 \\
\text { OLGB } \\
\text { corona } \\
\text { (center) }\end{array}$ & $\begin{array}{c}895 \mathrm{E} \\
2 \mathrm{R}-2 \\
6-10 \\
1 \\
\text { OLGB } \\
\text { ol psdm } \\
\text { (+tre) }\end{array}$ & $\begin{array}{c}895 \mathrm{E} \\
2 \mathrm{R}-2 \\
6-10 \\
1 \\
\text { OLGB } \\
\text { veinlet in plg }\end{array}$ & $\begin{array}{c}895 \mathrm{E} \\
2 \mathrm{R}-2 \\
6-10 \\
1 \\
\text { OLGB } \\
\text { corona } \\
\text { (center) }\end{array}$ & $\begin{array}{c}895 \mathrm{E} \\
6 \mathrm{R}-1 \\
15-17 \\
4 \mathrm{~A} \\
\mathrm{~GB} \\
\text { corona } \\
\text { (rim/plg) }\end{array}$ & $\begin{array}{c}895 \mathrm{E} \\
6 \mathrm{R}-1 \\
15-17 \\
4 \mathrm{~A} \\
\text { GB } \\
\text { plg psdm }\end{array}$ & $\begin{array}{c}895 \mathrm{E} \\
6 \mathrm{R}-1 \\
15-17 \\
4 \mathrm{~A} \\
\mathrm{~GB} \\
\text { veinlet in plg }\end{array}$ \\
\hline $\begin{array}{r}28.34 \\
0.07 \\
17.80 \\
2.00 \\
0.00 \\
15.56 \\
0.19 \\
23.12 \\
0.09 \\
0.08 \\
0.06 \\
0.01 \\
0.01 \\
11.85 \\
99.17\end{array}$ & $\begin{array}{r}27.73 \\
0.05 \\
17.41 \\
3.54 \\
0.00 \\
16.47 \\
0.18 \\
22.07 \\
0.10 \\
0.08 \\
0.06 \\
0.03 \\
0.02 \\
11.77 \\
99.50\end{array}$ & $\begin{array}{r}32.74 \\
0.00 \\
17.18 \\
0.01 \\
0.00 \\
4.88 \\
0.05 \\
31.78 \\
0.23 \\
0.14 \\
0.04 \\
0.03 \\
0.01 \\
12.64 \\
99.73\end{array}$ & $\begin{array}{r}30.88 \\
0.01 \\
20.53 \\
0.00 \\
0.00 \\
5.73 \\
0.11 \\
30.11 \\
0.08 \\
0.06 \\
0.02 \\
0.03 \\
0.01 \\
12.67 \\
100.24\end{array}$ & $\begin{array}{r}34.23 \\
0.03 \\
11.90 \\
0.00 \\
0.00 \\
16.21 \\
0.10 \\
25.28 \\
0.23 \\
0.19 \\
0.29 \\
0.12 \\
0.03 \\
12.08 \\
100.70\end{array}$ & $\begin{array}{r}27.59 \\
0.03 \\
21.47 \\
0.02 \\
0.13 \\
14.59 \\
0.15 \\
23.68 \\
0.19 \\
0.02 \\
0.01 \\
0.02 \\
0.01 \\
12.09 \\
100.00\end{array}$ & $\begin{array}{r}26.84 \\
0.01 \\
21.64 \\
0.01 \\
0.02 \\
17.22 \\
0.16 \\
21.49 \\
0.10 \\
0.03 \\
0.04 \\
0.01 \\
0.02 \\
11.87 \\
99.45\end{array}$ & $\begin{array}{r}29.32 \\
0.00 \\
20.26 \\
0.00 \\
0.00 \\
11.99 \\
0.10 \\
25.95 \\
0.18 \\
0.05 \\
0.03 \\
0.01 \\
0.02 \\
12.28 \\
100.20\end{array}$ & $\begin{array}{r}31.26 \\
0.01 \\
17.03 \\
0.02 \\
0.00 \\
11.94 \\
0.13 \\
26.92 \\
0.32 \\
0.03 \\
0.02 \\
0.02 \\
0.01 \\
12.26 \\
99.98\end{array}$ & $\begin{array}{r}28.31 \\
0.01 \\
20.36 \\
0.01 \\
0.14 \\
13.01 \\
0.10 \\
25.18 \\
0.01 \\
0.08 \\
0.03 \\
0.00 \\
0.00 \\
12.10 \\
99.33\end{array}$ & $\begin{array}{r}30.68 \\
0.00 \\
18.59 \\
0.00 \\
0.00 \\
9.46 \\
0.12 \\
27.93 \\
0.12 \\
0.05 \\
0.06 \\
0.04 \\
0.02 \\
12.33 \\
99.39\end{array}$ & $\begin{array}{r}29.49 \\
0.00 \\
20.28 \\
0.00 \\
0.00 \\
9.51 \\
0.11 \\
27.82 \\
0.10 \\
0.06 \\
0.06 \\
0.02 \\
0.02 \\
12.37 \\
99.85\end{array}$ & $\begin{array}{r}28.60 \\
0.00 \\
20.83 \\
0.00 \\
0.00 \\
11.40 \\
0.11 \\
25.98 \\
0.22 \\
0.09 \\
0.07 \\
0.02 \\
0.01 \\
12.21 \\
99.53\end{array}$ & $\begin{array}{r}28.25 \\
0.02 \\
21.25 \\
0.00 \\
0.00 \\
12.78 \\
0.15 \\
24.89 \\
0.17 \\
0.13 \\
0.10 \\
0.05 \\
0.02 \\
12.17 \\
99.98\end{array}$ \\
\hline \multicolumn{14}{|c|}{ Tetrahedral cations in 2:1 layer } \\
\hline $\begin{array}{l}2.87 \\
1.13\end{array}$ & $\begin{array}{l}2.82 \\
1.18\end{array}$ & $\begin{array}{l}2.82 \\
1.18\end{array}$ & $\begin{array}{l}3.11 \\
0.89\end{array}$ & $\begin{array}{l}2.92 \\
1.08\end{array}$ & $\begin{array}{l}3.40 \\
0.60\end{array}$ & $\begin{array}{l}2.74 \\
1.26\end{array}$ & $\begin{array}{l}2.71 \\
1.29\end{array}$ & $\begin{array}{l}2.86 \\
1.14\end{array}$ & $\begin{array}{l}3.06 \\
0.94\end{array}$ & $\begin{array}{l}2.80 \\
1.20\end{array}$ & $\begin{array}{l}2.98 \\
1.02\end{array}$ & $\begin{array}{l}2.86 \\
1.14\end{array}$ & $\begin{array}{l}2.81 \\
1.19\end{array}$ \\
\hline \multicolumn{14}{|c|}{ Octrahedral cations in 2:1 layer } \\
\hline $\begin{array}{l}1.64 \\
0.00 \\
0.00 \\
1.32 \\
0.02 \\
0.01 \\
2.98\end{array}$ & $\begin{array}{l}1.56 \\
0.00 \\
0.00 \\
1.40 \\
0.02 \\
0.01 \\
2.98\end{array}$ & $\begin{array}{l}2.39 \\
0.13 \\
0.00 \\
0.39 \\
0.00 \\
0.02 \\
2.93\end{array}$ & $\begin{array}{l}2.25 \\
0.21 \\
0.00 \\
0.45 \\
0.01 \\
0.01 \\
2.93\end{array}$ & $\begin{array}{l}1.53 \\
0.00 \\
0.00 \\
1.35 \\
0.01 \\
0.02 \\
2.90\end{array}$ & $\begin{array}{l}1.50 \\
0.25 \\
0.01 \\
1.21 \\
0.01 \\
0.01 \\
3.00\end{array}$ & $\begin{array}{l}1.23 \\
0.29 \\
0.00 \\
1.45 \\
0.01 \\
0.01 \\
3.00\end{array}$ & $\begin{array}{l}1.78 \\
0.19 \\
0.00 \\
0.98 \\
0.01 \\
0.01 \\
2.97\end{array}$ & $\begin{array}{l}1.95 \\
0.00 \\
0.00 \\
0.98 \\
0.01 \\
0.03 \\
2.96\end{array}$ & $\begin{array}{l}1.72 \\
0.19 \\
0.01 \\
1.08 \\
0.01 \\
0.00 \\
3.00\end{array}$ & $\begin{array}{l}2.05 \\
0.11 \\
0.00 \\
0.77 \\
0.01 \\
0.01 \\
2.95\end{array}$ & $\begin{array}{l}2.02 \\
0.17 \\
0.00 \\
0.77 \\
0.01 \\
0.01 \\
2.98\end{array}$ & $\begin{array}{l}1.80 \\
0.22 \\
0.00 \\
0.94 \\
0.01 \\
0.02 \\
2.99\end{array}$ & $\begin{array}{l}1.65 \\
0.25 \\
0.00 \\
1.05 \\
0.01 \\
0.01 \\
2.98\end{array}$ \\
\hline \multicolumn{14}{|c|}{ Octrahedral cations in interlayer (brucite layer) } \\
\hline $\begin{array}{l}1.84 \\
0.99 \\
0.16 \\
0.01 \\
3.00\end{array}$ & $\begin{array}{l}1.80 \\
0.92 \\
0.29 \\
0.00 \\
3.00\end{array}$ & $\begin{array}{l}2.11 \\
0.89 \\
0.00 \\
0.00 \\
3.00\end{array}$ & $\begin{array}{l}2.00 \\
1.00 \\
0.00 \\
0.00 \\
3.00\end{array}$ & $\begin{array}{l}2.21 \\
0.79 \\
0.00 \\
0.00 \\
3.00\end{array}$ & $\begin{array}{l}2.00 \\
0.99 \\
0.00 \\
0.00 \\
3.00\end{array}$ & $\begin{array}{l}2.00 \\
1.00 \\
0.00 \\
0.00 \\
3.00\end{array}$ & $\begin{array}{l}2.00 \\
1.00 \\
0.00 \\
0.00 \\
3.00\end{array}$ & $\begin{array}{l}1.98 \\
1.02 \\
0.00 \\
0.00 \\
3.00\end{array}$ & $\begin{array}{l}2.00 \\
1.00 \\
0.00 \\
0.00 \\
3.00\end{array}$ & $\begin{array}{l}2.00 \\
1.00 \\
0.00 \\
0.00 \\
3.00\end{array}$ & $\begin{array}{l}2.00 \\
1.00 \\
0.00 \\
0.00 \\
3.00\end{array}$ & $\begin{array}{l}2.00 \\
1.00 \\
0.00 \\
0.00 \\
3.00\end{array}$ & $\begin{array}{l}2.00 \\
1.00 \\
0.00 \\
0.00 \\
3.00\end{array}$ \\
\hline $\begin{array}{l}0.01 \\
0.01 \\
0.00\end{array}$ & $\begin{array}{l}0.01 \\
0.01 \\
0.00\end{array}$ & $\begin{array}{l}0.01 \\
0.01 \\
0.00\end{array}$ & $\begin{array}{l}0.01 \\
0.00 \\
0.00\end{array}$ & $\begin{array}{l}0.02 \\
0.06 \\
0.01\end{array}$ & $\begin{array}{l}0.00 \\
0.00 \\
0.00\end{array}$ & $\begin{array}{l}0.00 \\
0.01 \\
0.00\end{array}$ & $\begin{array}{l}0.01 \\
0.01 \\
0.00\end{array}$ & $\begin{array}{l}0.00 \\
0.00 \\
0.00\end{array}$ & $\begin{array}{l}0.01 \\
0.01 \\
0.00\end{array}$ & $\begin{array}{l}0.01 \\
0.01 \\
0.00\end{array}$ & $\begin{array}{l}0.01 \\
0.01 \\
0.00\end{array}$ & $\begin{array}{l}0.01 \\
0.010 \\
0.0\end{array}$ & $\begin{array}{l}0.01 \\
0.02 \\
0.01\end{array}$ \\
\hline \multicolumn{14}{|c|}{ Exchange vectors } \\
\hline $\begin{array}{r}-0.04 \\
0.02 \\
0.16 \\
0.73\end{array}$ & $\begin{array}{r}-0.12 \\
0.02 \\
0.29 \\
0.71\end{array}$ & $\begin{array}{r}-0.11 \\
0.07 \\
0.00 \\
0.92\end{array}$ & $\begin{array}{l}0.08 \\
0.07 \\
0.00 \\
0.90\end{array}$ & $\begin{array}{r}-0.40 \\
0.10 \\
0.00 \\
0.73\end{array}$ & $\begin{array}{l}0.26 \\
0.00 \\
0.00 \\
0.74\end{array}$ & $\begin{array}{l}0.29 \\
0.00 \\
0.00 \\
0.69\end{array}$ & $\begin{array}{l}0.14 \\
0.03 \\
0.00 \\
0.79\end{array}$ & $\begin{array}{r}-0.06 \\
0.04 \\
0.00 \\
0.80\end{array}$ & $\begin{array}{l}0.19 \\
0.00 \\
0.00 \\
0.78\end{array}$ & $\begin{array}{l}0.02 \\
0.05 \\
0.00 \\
0.84\end{array}$ & $\begin{array}{l}0.14 \\
0.02 \\
0.00 \\
0.84\end{array}$ & $\begin{array}{l}0.19 \\
0.01 \\
0.00 \\
0.80\end{array}$ & $\begin{array}{l}0.21 \\
0.02 \\
0.00 \\
0.78\end{array}$ \\
\hline
\end{tabular}

harzburgite), amount of alteration, or borehole is observed. Vein-serpentine separates generally yield lower $\delta^{18} \mathrm{O}$ values than whole-rock serpentine; 10 samples of vein serpentine have values from $2.2 \%$ to $3.4 \%$, with one sample of $4.0 \%$ (Table 11 ).

The dunite/harzburgite $(\mathrm{D} / \mathrm{H})$ ratios of the rock-serpentines range from $-69 \%$ to $-59 \%$, with one sample yielding a $\delta \mathrm{D}$ value of $-51 \%$ (Figs. 7 through 9; Table 11). The values lie within the range of published D/H ratios of oceanic serpentine (Wenner and Taylor, 1973; Sheppard, 1980; Sakai et al., 1990) and are similar to the values reported by Agrinier et al. (in press) from Hess Deep ultramafic rocks. The $\mathrm{D} / \mathrm{H}$ ratios of vein-serpentine separates scatter over a range of $22 \%$, with $\delta \mathrm{D}$ values from $-46 \%$ o to $-68 \%$ o. With the exception of two samples, the vein-serpentine are generally slightly enriched in deuterium and are depleted in ${ }^{18} \mathrm{O}$ compared to the host rock serpentines.

\section{Fluid Sources and Estimates of Serpentinization Temperatures}

A depletion in ${ }^{18} \mathrm{O}$ relative to unaltered oceanic crust is characteristic of the Hess Deep gabbros and peridotites and clearly reflects hydrothermal alteration by fluids with a dominant seawater component at temperatures greater than $250^{\circ}-350^{\circ} \mathrm{C}$ (Lécuyer et al., this volume; Früh-Green et al., this volume; Agrinier et al., in press). However, if we consider the empirical serpentine-water calibration curve of Wenner and Taylor (1973), the hydrogen and oxygen isotope compositions of the Hess Deep samples cannot solely reflect the interaction with unaltered seawater, having $\delta \mathrm{D}$ and $\delta^{18} \mathrm{O}$ values of approximately $0 \%$. In particular, for a temperature range of $200^{\circ}-400^{\circ} \mathrm{C}$ and assuming unaltered seawater, the empirical fractionation curves of Wenner and Taylor (1973) predict the formation of serpentine with $\delta \mathrm{D}$ values of approximately $-20 \%$ o to $-35 \%$, which are at least $25 \%$ - $35 \%$ o higher than our data. The petrographic analysis of the Site 895 ultramafic rocks shows a considerable variation in the degree of alteration on a thin section scale, suggesting that the fluid/rock (F/R) ratios during metamorphism could have been low. A minimum $\mathrm{F} / \mathrm{R}$ ratio of 0.3 atom percent is dictated by the stoichiometric amount of $\mathrm{H}_{2} \mathrm{O}$ in the serpentine structure. The effects of temperature and fluid/rock ratios on hydrogen and oxygen ratios can be evaluated by considering the open system model for serpentinization proposed by Sakai et al. (1990), as shown in Figure 7. This model predicts that serpentinization at relatively low fluid/rock ratio results in more deuterium-rich compositions, and would thus increase the discrepancy between predicted and measured values of the Hess Deep samples (Fig. 7). Consequently, the isotope ratios of the Site 895 serpentines must reflect an additional non-seawater component with distinctly lower D/H ratios, and suggest that magmatic or metamorphic volatiles may have played a significant role in the hydrothermal evolution at Hess Deep.

Three magnetite-serpentine mineral pairs were separated and measured for $\delta^{18} \mathrm{O}$, in order to further constrain serpentinization temperatures. The purity of the magnetite $(\mathrm{mt})$ separates was $>95 \%$. Minor amounts of chromite impurities (which are assumed to have pos- 


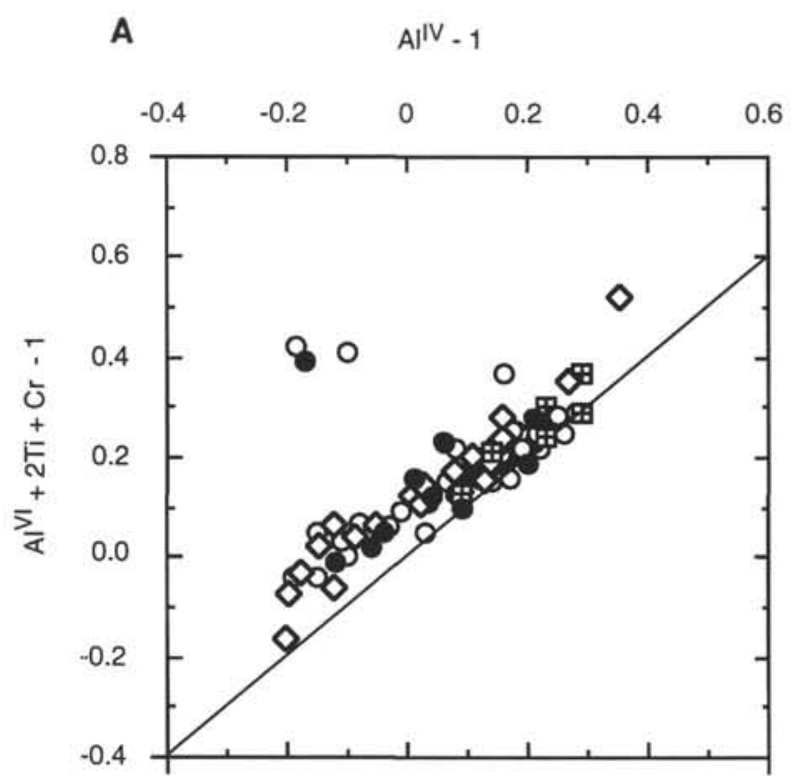

$\diamond$ Troctolite

田 Coarse-grained diallage gabbro
B $\mathrm{Mg} /\left(\mathrm{Mg}+\mathrm{Fe}_{\text {total }}\right)$

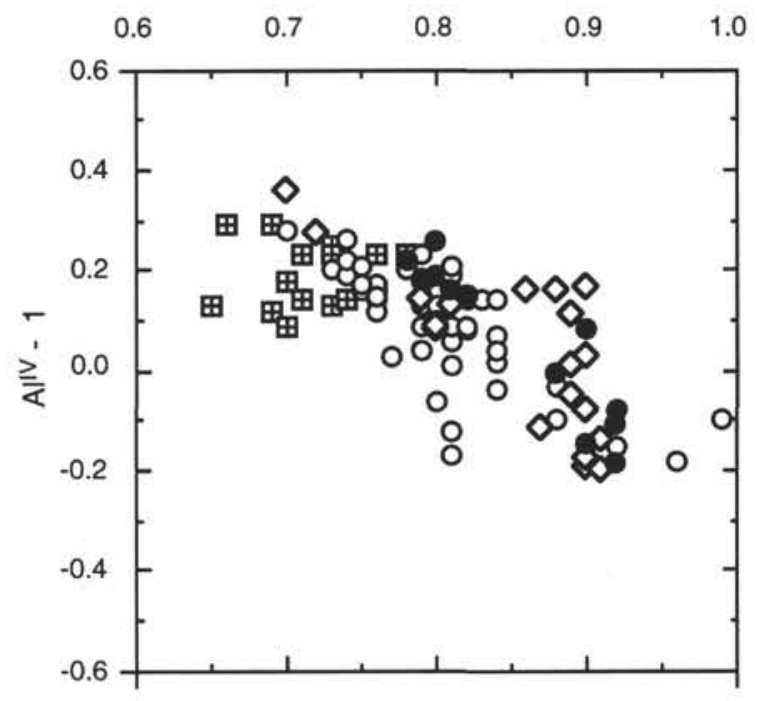

- Moderately altered olivine gabbro

O Highly to pervasively altered olivine gabbro

Figure 6. Chlorite compositions of Site 895 troctolites, olivine gabbros, and coarse-grained diallage gabbro. A. Tschermak substitutions $\left(\mathrm{Al}^{\mathrm{Vl}} \mathrm{Al}^{\mathrm{Vl}} \mathrm{Mg}_{-1} \mathrm{Si}_{-1}\right.$, $\mathrm{CrAl}^{\mathrm{Vl}} \mathrm{Mg}_{-1} \mathrm{Si}_{-1}$, and $\left.\mathrm{TiAl}_{2}{ }_{2} \mathrm{Mg}_{-1} \mathrm{Si}_{-2}\right)$ in chlorite with clinochlore-chamosite, $(\mathrm{Mg}, \mathrm{Fe})_{5} \mathrm{AlSi}_{3} \mathrm{AlO}_{10}(\mathrm{OH})_{8}$, as the origin. Variations in compositions along the 1:1 line from the origin represent the combined Tschermak exchange in chlorite. Compositions plotting above this line indicate dioctahedral substitution with octahedral vacancies (Laird, 1988). B. Tetrahedral-site cation distribution in chlorite, plotted as a function of Tschermak substitutions and Mg to Fe ratios (Laird, 1988).

itive $\delta^{18} \mathrm{O}$ values) could slightly decrease the $\Delta_{\text {serp-mt }}$ and would insignificantly increase the calculated temperatures. The $\delta^{18} \mathrm{O}$ values of magnetite range from $-0.9 \%$ to $1.2 \%$ (Table 11 ).

Empirical serpentine-water $\left(\right.$ serp- $\left.\mathrm{H}_{2} \mathrm{O}\right)$ and serpentine-magnetite (serp-mt) oxygen isotope fractionation curves were proposed by Wenner and Taylor (1971). These curves were based on the assumption that chlorite and serpentine are essentially isotopically indistinguishable and were calculated by combining (1) oxygen isotope fractionations measured in natural, regional metamorphic samples, (2) preliminary experimental data of the quartz-water $\left(\mathrm{qtz}-\mathrm{H}_{2} \mathrm{O}\right)$ oxygen isotope fractionation (based on personal communications from Clayton, O'Neil, and Mayeda), and (3) experimentally determined fractionation factors for muscovite-water (O'Neil and Taylor, 1969). In this study, we have used the fractionation relationships discussed in the original work of Wenner and Taylor (1971), but propose two different ways in which the curve can be calculated. The first possibility takes into account differences between the unpublished data and the published quartz-water fractionation curve of Clayton et al. (1972), as reported in Friedman and O'Neil (1977). Using the curve $10^{3} \ln \alpha_{q 1 z-\mu_{0}}=3.38 \times 10^{6} / \mathrm{T}^{2}-2.9$ (as given in Friedman and O'Neil, 1977), we obtain the following equation for the serp-mt fractionation curve:

$$
10^{3} \ln \alpha_{\text {serp-mt }}=1.42 \times 10^{6} / \mathrm{T}^{2}+0.93
$$

The second possibility uses an empirical calibration of quartz-water recently published by Sharp and Kirschner (1994): $10^{3} \operatorname{In}$ $\alpha_{\mathrm{q} t \mathrm{z}-\mathrm{H}_{2} \mathrm{O}}=3.65 \times 10^{6} / \mathrm{T}^{2}-2.9$. This calibration was derived from natural data in which isotopic equilibrium could be shown. We prefer this calibration because the use of natural equilibrium fractionations avoids uncertainties due to kinetic effects during recrystallization in direct-exchange experiments (see discussion in Sharp and Kirschner, 1994), and yields more geologically reasonable temperatures, particularly at low temperatures. Thus, using this curve, we obtain the following equations for the serp- $\mathrm{H}_{2} \mathrm{O}$ and the serp-mt oxygen isotope fractionations:

$$
10^{3} \ln \alpha_{\text {sepp- } \mathrm{H}_{2} \mathrm{O}}=1.51 \times 10^{6} / \mathrm{T}^{2}-4.57 \text {; }
$$

and

$$
10^{3} \ln \alpha_{\text {serp-mt }}=1.81 \times 10^{6} / \mathrm{T}^{2}+1.41 .
$$

Curves (2) and (3) yield temperatures that are similar to those calculated from the original curve published by Wenner and Taylor (1971) and is consistent with data from metamorphic serpentines in the Malenco ophicarbonates of northern Italy (D. Pozzorini, pers. comm., 1994).

By applying equation (1) above, we obtain temperatures of $297^{\circ}$, $386^{\circ}$, and $376^{\circ} \mathrm{C} \pm 50^{\circ} \mathrm{C}$ for the three serpentine-magnetite pairs. Equation (3) yields temperatures of $410^{\circ}, 533^{\circ}$, and $518^{\circ} \mathrm{C} \pm 50^{\circ} \mathrm{C}$, respectively. A serpentinization temperature of $325^{\circ} \mathrm{C} \pm 50^{\circ} \mathrm{C}$, based on the original curve proposed by Wenner and Taylor (1971), has been reported by Agrinier et al. (in press) for one sample previously collected at Hess Deep. Such high temperatures are considered to be at or above the stability limits of lizardite and chrysotile polytypes, favoring the high-temperature antigorite phase (e.g., Evans and Trommsdorff, 1970; Moody, 1979; Chernosky et al., 1988), which is clearly less abundant in the examined samples. However, a number of factors could allow the persistence of lizardite up to relatively high temperatures and are discussed in detail below. Although the mineralogical and stable isotope data of both ultramafic and mafic rocks in- 
Table 10. Representative compositions of calc-silicates and thomsonite in Site 895 mafic rocks.

\begin{tabular}{|c|c|c|c|c|c|c|c|c|c|c|c|c|c|c|c|c|c|c|}
\hline \multirow{7}{*}{$\begin{array}{l}\text { Hole: } \\
\text { Core, section: } \\
\text { Interval }(\mathrm{cm}): \\
\text { Piece: } \\
\text { Lithology: } \\
\text { Association: }\end{array}$} & \multicolumn{10}{|c|}{ Prehnite } & \multicolumn{3}{|c|}{ Hydrogrossular } & \multicolumn{5}{|c|}{ Thomsonite } \\
\hline & 895D & 895D & 895D & $895 \mathrm{D}$ & 895D & 895D & $895 \mathrm{E}$ & $895 \mathrm{E}$ & $895 \mathrm{E}$ & $895 \mathrm{E}$ & 895D & 895D & 895D & $895 \mathrm{E}$ & $895 \mathrm{E}$ & $895 \mathrm{E}$ & $895 \mathrm{E}$ & $895 \mathrm{E}$ \\
\hline & $7 \mathrm{R}-1$ & $7 \mathrm{R}-1$ & $8 \mathrm{R}-2$ & $8 \mathrm{R}-2$ & $8 \mathrm{R}-2$ & $8 \mathrm{R}-2$ & $2 \mathrm{R}-1$ & $2 \mathrm{R}-1$ & $6 \mathrm{R}-1$ & $6 \mathrm{R}-1$ & $7 \mathrm{R}-1$ & $7 R-1$ & $7 \mathrm{R}-1$ & $2 \mathrm{R}-1$ & $2 \mathrm{R}-2$ & & & $6 \mathrm{R}-1$ \\
\hline & $81-84$ & $81-84$ & $14-19$ & $14-19$ & $14-19$ & $14-19$ & $91-95$ & $91-95$ & $15-17$ & $15-17$ & $81-84$ & $81-84$ & $81-84$ & 91-95 & $6-10$ & $6-10$ & $15-17$ & $15-17$ \\
\hline & 13 & 13 & 2 & $2^{2}$ & 2 & 2 & 14 & 14 & $4 \mathrm{~A}$ & $4 \mathrm{~A}$ & 13 & 13 & 13 & 14 & 1 & 1 & $4 \mathrm{~A}$ & $4 \mathrm{~A}$ \\
\hline & TRO & TRO & OLGB & OLGB & OLGB & OLGB & OLGB & OLGB & GB & GB & TRO & TRO & TRO & OLGB & OLGB & OLGB & GB & GB \\
\hline & plg psdm & plg psdm & plg psdm & $\begin{array}{c}\text { plg psdm } \\
\text { (brn) }\end{array}$ & vein & vein & vein & vein & vein & $\begin{array}{l}\text { vein (brn, } \\
\text { xcut, cpx) }\end{array}$ & plg psdm & plg psdm & plg psdm & plg psdm & plg psdm & vein & plg psdm & vein \\
\hline $\mathrm{SiO}_{2}$ & 43.23 & 42.32 & 42.80 & 41.41 & 43.35 & 42.51 & 43.21 & 43.31 & 42.94 & 43.38 & 28.13 & 31.52 & 33.30 & 37.96 & 39.73 & 37.92 & 38.59 & 37.40 \\
\hline $\mathrm{TiO}_{2}^{2}$ & 0.03 & 0.04 & 0.02 & 0.00 & 0.01 & 0.01 & 0.00 & 0.03 & 0.02 & 0.00 & 0.01 & 0.02 & 0.03 & 0.00 & 0.00 & 0.01 & 0.01 & 0.01 \\
\hline $\mathrm{Al}_{2} \mathrm{O}_{3}$ & 25.10 & 24.59 & 24.63 & 25.48 & 24.54 & 24.70 & 24.52 & 24.70 & 24.89 & 24.73 & 22.68 & 22.25 & 28.42 & 31.07 & 30.05 & 31.47 & 30.35 & 30.70 \\
\hline $\mathrm{Cr}_{2} \mathrm{O}_{3}$ & 0.01 & 0.00 & 0.02 & 0.00 & 0.00 & 0.00 & 0.02 & 0.00 & 0.00 & 0.00 & 0.00 & 0.00 & 0.00 & 0.00 & 0.00 & 0.00 & 0.00 & 0.00 \\
\hline $\mathrm{Fe}_{2} \mathrm{O}_{3}$ & 0.20 & 0.39 & 0.14 & 0.02 & 0.06 & 0.01 & 0.06 & 0.03 & 0.17 & 0.05 & 0.00 & 0.00 & 0.00 & 0.03 & 0.06 & 0.21 & 0.31 & 0.10 \\
\hline $\mathrm{FeO}$ & 0.00 & 0.00 & 0.00 & 0.00 & 0.00 & 0.00 & 0.00 & 0.00 & 0.00 & 0.00 & 0.39 & 1.03 & 0.36 & 0.00 & 0.00 & 0.00 & 0.00 & 0.00 \\
\hline $\mathrm{MnO}$ & 0.02 & 0.05 & 0.03 & 0.02 & 0.01 & 0.00 & 0.08 & 0.00 & 0.01 & 0.02 & 0.06 & 0.07 & 0.04 & 0.00 & 0.00 & 0.02 & 0.01 & 0.02 \\
\hline $\mathrm{NiO}$ & 0.00 & 0.02 & 0.05 & 0.00 & 0.00 & 0.04 & 0.01 & 0.00 & 0.00 & 0.00 & 0.04 & 0.00 & 0.04 & 0.00 & 0.00 & 0.00 & 0.01 & 0.03 \\
\hline $\mathrm{MgO}$ & 0.05 & 0.33 & 0.01 & 0.04 & 0.02 & 0.03 & 0.01 & 0.01 & 0.36 & 0.05 & 0.41 & 0.26 & 0.21 & 0.00 & 0.00 & 0.03 & 0.13 & 0.03 \\
\hline $\mathrm{CaO}$ & 26.42 & 26.06 & 27.09 & 27.15 & 27.32 & 27.04 & 27.14 & 27.14 & 27.13 & 27.55 & 39.23 & 38.04 & 29.96 & 13.51 & 12.68 & 13.43 & 13.32 & 13.75 \\
\hline $\mathrm{Na}_{2} \mathrm{O}$ & 0.02 & 0.11 & 0.04 & 0.04 & 0.19 & 0.17 & 0.07 & 0.09 & 0.10 & 0.06 & 0.01 & 0.03 & 0.04 & 3.85 & 2.64 & 2.98 & 3.94 & 3.76 \\
\hline $\mathrm{K}_{2} \mathrm{O}$ & 0.00 & 0.01 & 0.01 & 0.02 & 0.00 & 0.02 & 0.00 & 0.00 & 0.02 & 0.00 & 0.00 & 0.00 & 0.01 & 0.00 & 0.00 & 0.00 & 0.02 & 0.04 \\
\hline $\mathrm{Cl}$ & 0.02 & 0.04 & 0.00 & 0.01 & 0.00 & 0.02 & 0.01 & 0.01 & 0.01 & 0.00 & 0.03 & 0.05 & 0.03 & 0.01 & 0.01 & 0.00 & 0.00 & 0.00 \\
\hline $\mathrm{H}_{2} \mathrm{O}$ & 4.34 & 4.27 & 4.32 & 4.28 & 4.35 & 4.30 & 4.33 & 4.35 & 4.35 & 4.37 & 3.79 & 3.93 & $\begin{array}{l}0.073 \\
4.07\end{array}$ & 13.41 & 13.38 & 13.41 & 13.45 & 13.28 \\
\hline Total & 99.44 & 98.22 & 99.14 & 98.46 & 99.85 & 98.88 & 99.47 & 99.68 & 100.00 & 100.20 & 94.79 & 97.21 & 96.52 & 99.85 & 98.56 & 99.49 & 100.13 & 99.11 \\
\hline $\mathrm{Si}$ & 5.96 & 5.92 & 5.94 & 5.80 & 5.97 & 5.92 & 5.97 & 5.97 & 5.91 & 5.96 & 4.44 & 4.80 & 4.89 & 20.37 & 21.36 & 20.35 & 20.65 & 20.26 \\
\hline $\mathrm{Ti}$ & 0.00 & 0.00 & 0.00 & 0.00 & 0.00 & 0.00 & 0.00 & 0.00 & 0.00 & 0.00 & 0.00 & 0.00 & 0.00 & 0.00 & 0.00 & 0.00 & 0.00 & 0.01 \\
\hline $\mathrm{Al}^{\mathrm{IV}}$ & 2.04 & 2.08 & 2.06 & 2.20 & 2.03 & 2.08 & 2.03 & 2.03 & 2.09 & 2.04 & 1.56 & 1.20 & 1.11 & 19.63 & 18.64 & 19.65 & 19.14 & 19.60 \\
\hline $\mathrm{Al}^{\mathrm{vI}}$ & 2.04 & 1.98 & 1.97 & 2.00 & 1.96 & 1.98 & 1.97 & 1.99 & 1.95 & 1.96 & 2.65 & 2.79 & 3.82 & 0.01 & 0.40 & 0.26 & 0.00 & 0.00 \\
\hline $\mathrm{Fe}^{3+}$ & 0.02 & 0.04 & 0.01 & 0.00 & 0.01 & 0.00 & 0.01 & 0.00 & 0.02 & 0.00 & 0.00 & 0.00 & 0.00 & 0.01 & 0.02 & 0.09 & 0.13 & 0.04 \\
\hline $\mathrm{Fe}^{2+}$ & 0.00 & 0.00 & 0.00 & 0.00 & 0.00 & 0.00 & 0.00 & 0.00 & 0.00 & 0.00 & 0.05 & 0.13 & 0.04 & 0.00 & 0.00 & 0.00 & 0.00 & 0.00 \\
\hline $\mathrm{Mn}$ & 0.00 & 0.01 & 0.00 & 0.00 & 0.00 & 0.00 & 0.01 & 0.00 & 0.00 & 0.00 & 0.01 & 0.01 & 0.01 & 0.00 & 0.00 & 0.01 & 0.00 & 0.01 \\
\hline $\mathrm{Ni}$ & 0.00 & 0.00 & 0.01 & 0.00 & 0.00 & 0.00 & 0.00 & 0.00 & 0.00 & 0.00 & 0.01 & 0.00 & 0.00 & 0.00 & 0.00 & 0.00 & 0.00 & 0.01 \\
\hline $\mathrm{Mg}$ & 0.01 & 0.07 & 0.00 & 0.01 & 0.00 & 0.01 & 0.00 & 0.00 & 0.07 & 0.01 & 0.10 & 0.06 & 0.05 & 0.00 & 0.00 & 0.02 & 0.10 & 0.03 \\
\hline $\mathrm{Ca}$ & 3.90 & 3.91 & 4.03 & 4.07 & 4.03 & 4.04 & 4.02 & 4.01 & 4.00 & 4.05 & 6.63 & 6.21 & 4.72 & 7.77 & 7.30 & 7.72 & 7.63 & 7.98 \\
\hline $\mathrm{Na}$ & 0.01 & 0.03 & 0.01 & 0.01 & 0.05 & 0.0 & 0.0 & 0.02 & 0.03 & 0.0 & 0.00 & 0.01 & 0.01 & 4.01 & 2.75 & 3.10 & 4.09 & 3.94 \\
\hline $\mathrm{K}$ & 0.00 & 0.00 & 0.0 & 0.00 & 0.00 & 0.00 & 0.0 & 0.00 & 0.00 & 0.0 & 0.0 & 0.0 & 0.0 & 0.00 & 0.00 & 0.0 & 0.01 & 0.03 \\
\hline $\mathrm{Cl}$ & 0.0 & 0.0 & 0. & 0.0 & 0.00 & 0.0 & 0.0 & 0.0 & 0.0 & 0. & 0.01 & 0.01 & 0.01 & 0.01 & 0.01 & 0.00 & 0.00 & 0.00 \\
\hline $\mathrm{H}$ & 3.99 & 3.99 & 4.00 & 4.00 & 4.00 & 4.00 & 4.00 & 4.00 & 4.00 & 4.00 & 3.99 & 3.99 & 3.99 & 47.99 & 47.99 & 48.00 & 48.00 & 48.00 \\
\hline Sum cations & 13.99 & 14.04 & 14.04 & 14.10 & 14.06 & 14.07 & 14.03 & 14.03 & 14.08 & 14.05 & 15.46 & 15.21 & 14.65 & 51.81 & 50.48 & 51.20 & 51.76 & 51.90 \\
\hline $\mathrm{xMg}_{\text {(Fetox) }}$ & 0.35 & 0.63 & 0.11 & 0.76 & 0.39 & 0.83 & 0.32 & 0.32 & 0.81 & 0.66 & 0.66 & 0.31 & 0.51 & 0.23 & 0.11 & 0.22 & 0.45 & 0.39 \\
\hline
\end{tabular}

Notes: Prehnite formulas calculated on the basis of 24 oxygens and assuming total $\mathrm{Fe}$ as $\mathrm{Fe}^{3+}$. Hydrogrossular formulas calculated on the basis of 24 oxygens and assuming total $\mathrm{Fe}$ as $\mathrm{Fe}^{2+}$. Thomsonite formulas calculated on the basis of 104 oxygens ( 80 framework oxygens), assuming total $\mathrm{Fe}$ as $\mathrm{Fe}^{3+}, \mathrm{xMg}(\mathrm{Fetot})=\mathrm{Mg} /(\mathrm{Mg}+\mathrm{Fe}$ total $)$. Mineral abbreviations as in Tables 1 through 9. 
Table 11. Stable isotope ratios of mineral separates and veins from shallow mantle sequences at Site 895 .

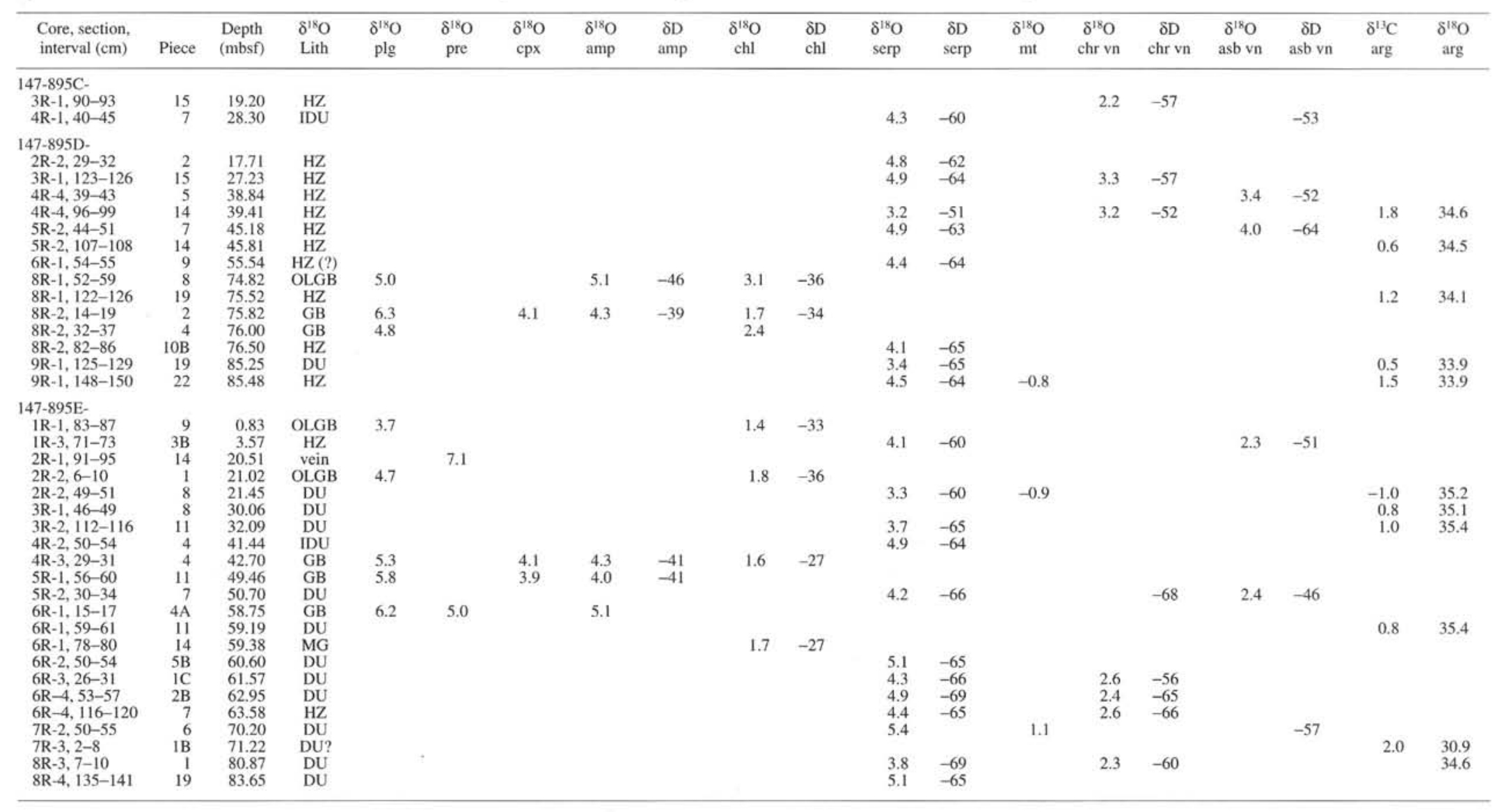

Note: All values in \%o relative to SMOW for $\delta^{18} \mathrm{O}$ and $\delta \mathrm{D}$, and relative to PDP for $\delta^{13} \mathrm{C}$. Rock and mineral abbreviations as in Table 1 . 


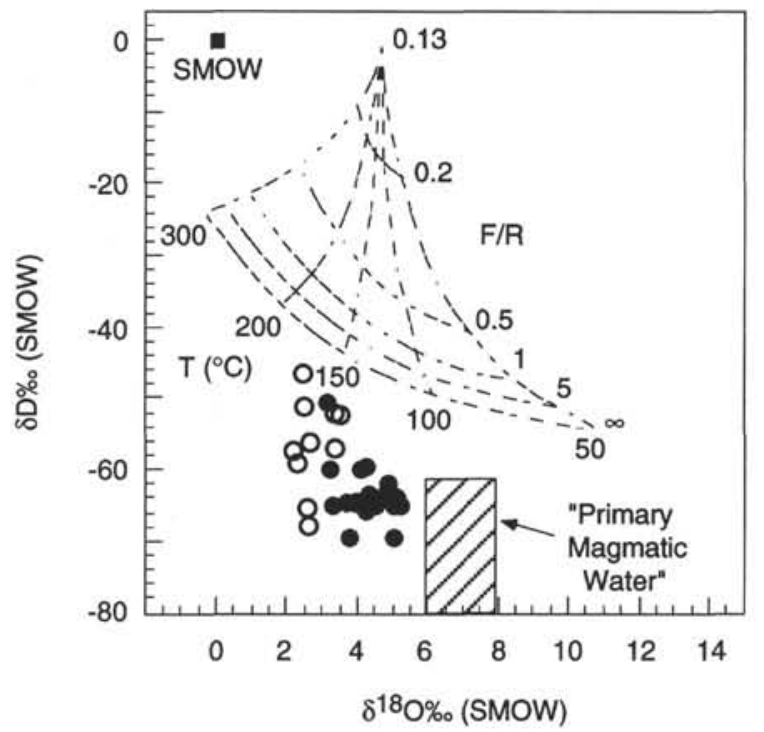

Figure 7. Hydrogen and oxygen isotope ratios of the Site 895 serpentine minerals. The open system model of Sakai et al. (1990, dashed lines; modified after Wenner and Taylor, 1973) predicts oxygen and hydrogen isotope ratios of serpentine equilibrated with seawater (SMOW) at different temperatures and water/rock ratios. The Site 895 serpentines plot distinctly outside this field. Rock-serpentine separates are represented by solid circles; vein serpentine separates are open circles.

dicate a high-temperature metamorphic event, these calculated temperatures can only be considered rough estimates due to the uncertainties in available oxygen isotope fractionation factors of serpentine and possible isotopic disequilibrium. In the absence of better calibrations and for purposes of subsequent discussion, we have taken an approximate average of the different calculated temperatures and propose a temperature of $400^{\circ} \mathrm{C} \pm 50^{\circ}$ as an estimate of the temperature of serpentinization at Hess Deep.

By applying the modified serpentine-water fractionation curve given by equation (2) above, and assuming a serpentinization temperature of $400^{\circ} \mathrm{C}$, the calculated $\delta^{18} \mathrm{O}$ value of the water in equilibrium with serpentine would range from $4.4 \%$ to $6.6 \%$. These values provide further limits on the isotopic composition of the hydrothermal fluids and suggests that the serpentine minerals equilibrated with a mixture of hydrothermally altered seawater having a $\delta^{18} \mathrm{O}$ of $0 \%-3 \%$ with a magmatic- or metamorphic-derived fluid having a consistently heavier $\delta^{18} \mathrm{O}$ value of $5 \%$ o to $8 \%$. This interpretation is consistent with the negative $\delta \mathrm{D}$ values of the measured samples $(\delta \mathrm{D}=-51 \%$ to $-69 \%$ ), which cannot solely be attained by equilibrium with altered seawater.

In Hole 895E, a general depth-dependent trend in both $\delta^{18} \mathrm{O}$ and $\delta \mathrm{D}$ of the rock serpentine minerals is defined (Fig. 9; Table 11). Over the penetration distance of approximately $84 \mathrm{~m}$, the $\delta^{18} \mathrm{O}$ shifts linearly from a value of approximately $3.5 \%$ at the top to approximately $5.0 \%$ at the bottom, whereas the $\delta \mathrm{D}$ values display an opposite trend, and are less negative at the top (approximately -60\%o) than at the bottom (approximately $-68 \%$ ). This depth correlation is observed only in Hole 895E. In Hole 895D (Fig. 8), a weakly defined trend is observed but is not considered significant because of the scattered stable magnetic inclinations, which suggest that the recovered cores may have penetrated several rotated blocks (see Gillis, Mével, Allan, et al., 1993; Kelso et al., this volume). We suggest that the $\delta \mathrm{D}$ and the $\delta^{18} \mathrm{O}$ values in Hole $895 \mathrm{E}$ have been produced by a gradient in the isotopic composition of the hydrothermal fluids. In fact, considering the minimal penetration distance, a temperature gradient effect is rather improbable. By applying equation (2) above and assuming an aver- age value $\delta^{18} \mathrm{O}$ value of $5.5 \%$ for the serpentinizing fluid and infinite fluid/rock ratios, the measured serpentine compositions (Fig. 9; Table 11) would correspond to temperatures of approximately $495^{\circ} \mathrm{C}$ at the top of the hole and $335^{\circ} \mathrm{C}$ at the bottom of the hole. This would indicate an inverse temperature gradient of approximately $1.9^{\circ} \mathrm{C} / \mathrm{m}$ over the penetration depth of Hole 895E.

If we refer to the open system model of Sakai et al. (1990; Fig. 7), minor differences in the fluid/rock ratios during alteration could have produced the $\delta \mathrm{D}$ trend, which would indicate higher fluid/rock ratio and degree of alteration downhole and correlates with the slight decrease of the bulk density of the rocks downhole (see Gillis, Mével, Allan, et al., 1993, Site 895, fig. 58). However, this scenario is contrary to the oxygen isotope ratios, which would indicate higher fluid/ rock ratio at the top of the hole. A gradient in the isotopic composition of the serpentinizing fluids, having a higher contribution of a deuterium-depleted component downhole, is therefore the most reasonable interpretation of the observed trends. A preferred fluid flux direction (i.e., lateral versus vertical) producing this gradient cannot be determined with the present data.

The relatively constant oxygen isotope ratios, but highly variable hydrogen isotope ratios of the vein serpentine, preclude a constant isotopic composition of the hydrothermal fluids. The relatively positive $\delta \mathrm{D}$ values of the vein serpentines relative to the host rock serpentines may reflect episodic hydrothermal activity and precipitation from more seawater-dominated hydrothermal fluids. Assuming, constant compositions of the infiltrating fluids, $\mathrm{O}$-isotope fractionations would predict higher temperature conditions for the veins than for the host rocks.

Deuterium depletion is common in oceanic and ophiolite serpentines and the origin of the low-D fluids remains controversial (e.g., Wenner and Taylor, 1973; Heaton and Sheppard, 1977; Sheppard, 1980; Kyser and Kerrich, 1991; Burkhard and O'Neil, 1988; Sakai, et al., 1990; Früh-Green et al., 1990). Processes leading to deuteriumdepleted serpentines may include isotopic disequilibrium conditions during serpentinization, exchange with magmatic volatiles, or infiltration of aqueous fluids released during dehydration reactions of oceanic crust that was previously hydrated at low temperatures (e.g., Wenner and Taylor, 1973; Sheppard, 1980; Sakai et al., 1990). Mass balance considerations predict that the $\mathrm{D} / \mathrm{H}$ ratios of the Hess Deep serpentinites would require that at least $20 \%$ of the serpentinizing fluid had a value of $-80 \%$ or less. Considering the fact that the serpentine structure contains 12 or more wt $\% \mathrm{H}_{2} \mathrm{O}$ and mafic magmas contain approximately $0.2-0.4 \mathrm{wt} \% \mathrm{H}_{2} \mathrm{O}$, this would require an unrealistically large contribution of magmatic water. Metamorphic water released during the dehydration of oceanic crust, previously altered through low-temperature seawater-rock interactions, could be a more tenable source of deuterium-depleted fluids (Sheppard, 1980). However, at both Sites 894 and 895 , no petrological evidence has been found that indicates either an early lower temperature phase of seawater-hydrothermal alteration or significant dehydration reactions during the tectono-metamorphic evolution of the Hess Deep. Therefore, other chemical processes that lead to hydrogen fractionation during alteration of the oceanic lithosphere should be considered. For instance, a strong $\mathrm{D} / \mathrm{H}$ fractionation associated with production of molecular hydrogen from $\mathrm{OH}^{-}$has been documented in an experimental study by Freund et al. (1982). They have shown that the process of dissolution of water in oxides such as $\mathrm{MgO}$ and $\mathrm{CaO}$ not only produces $\mathrm{OH}^{-}$ions, but molecular hydrogen forms from a certain fraction of the dissolved hydroxyl ions without transition metal cations acting as reducing agents.

The process of serpentinization of ultramafic rocks involves complex chemical equilibria and is sensitive to changes in redox conditions (Trommsdorff and Evans, 1977; Seyfried and Dibble, 1980; Frost 1985). Serpentinization is commonly considered to be accompanied by reducing conditions with extremely low oxygen fugacities $\left(f \mathrm{O}_{2}\right)$, whereby the formation of magnetite or hematite during serpen- 


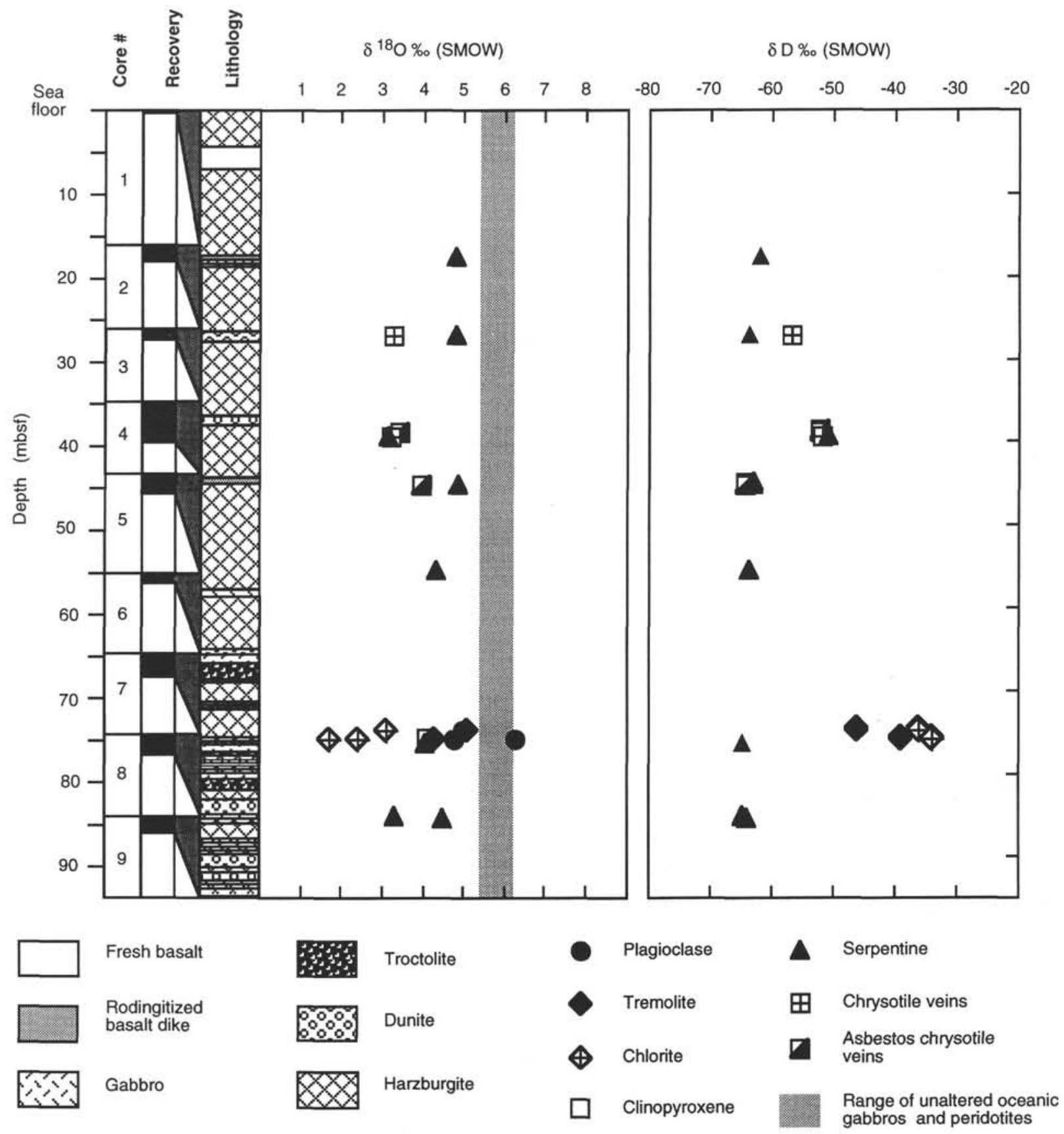

Olivine gabbro

Figure 8. Variation in hydrogen and oxygen isotope ratios as a function of depth in Hole 895D.

tinization releases hydrogen (e.g., Frost 1985). Furthermore, oxygen fugacity will also greatly affect the chemical composition of any carbon-bearing fluid. As a fluid penetrates into an ultramafic rock and causes serpentinization, conditions of increasingly lower $\mathrm{fO}_{2}$ will prevail at the serpentinization front and any $\mathrm{CO}_{2}$ in the fluid would be converted to methane. Since fractionations between hydrogen (gas) and water (vapor) as well as between methane and water are extremely large, the formation of even small quantities of hydrogen or methane in a metasomatic fluid could have important geochemical consequences. Thus, the presence of $\mathrm{H}_{2}$ or $\mathrm{CH}_{4}$ produced by $\mathrm{CO}_{2}$-reduction in the serpentinizing fluid in a rock-dominated system could result in the $\mathrm{H}$-rich serpentine compositions observed in many oceanic serpentinites and ophiolites. The production of $\mathrm{H}_{2}$ and $\mathrm{CH}_{4}$ during serpentinization is also consistent with an increasing number of discoveries of methane and hydrogen anomalies associated with ultramafic outcrops on the MAR and gas seeps in ophiolites (e.g., Abrajano et al., 1988; Charlou et al., 1991a, 1991b; Rona et al., 1992; Bougault, et al., 1993; Charlou and Donval, 1993), and is compatible with the presence of methane in fluid inclusions in gabbroic rocks from ODP Hole 735B at the Southwest Indian Ridge (Kelley, 1994; Kelley et al., 1994; Kelley and Früh-Green, 1995).

\section{$\mathrm{CO}_{2}$ in Serpentinized Peridotites}

The results of the thermal decomposition experiments carried out on two pervasively serpentinized peridotites are given in Table 12 


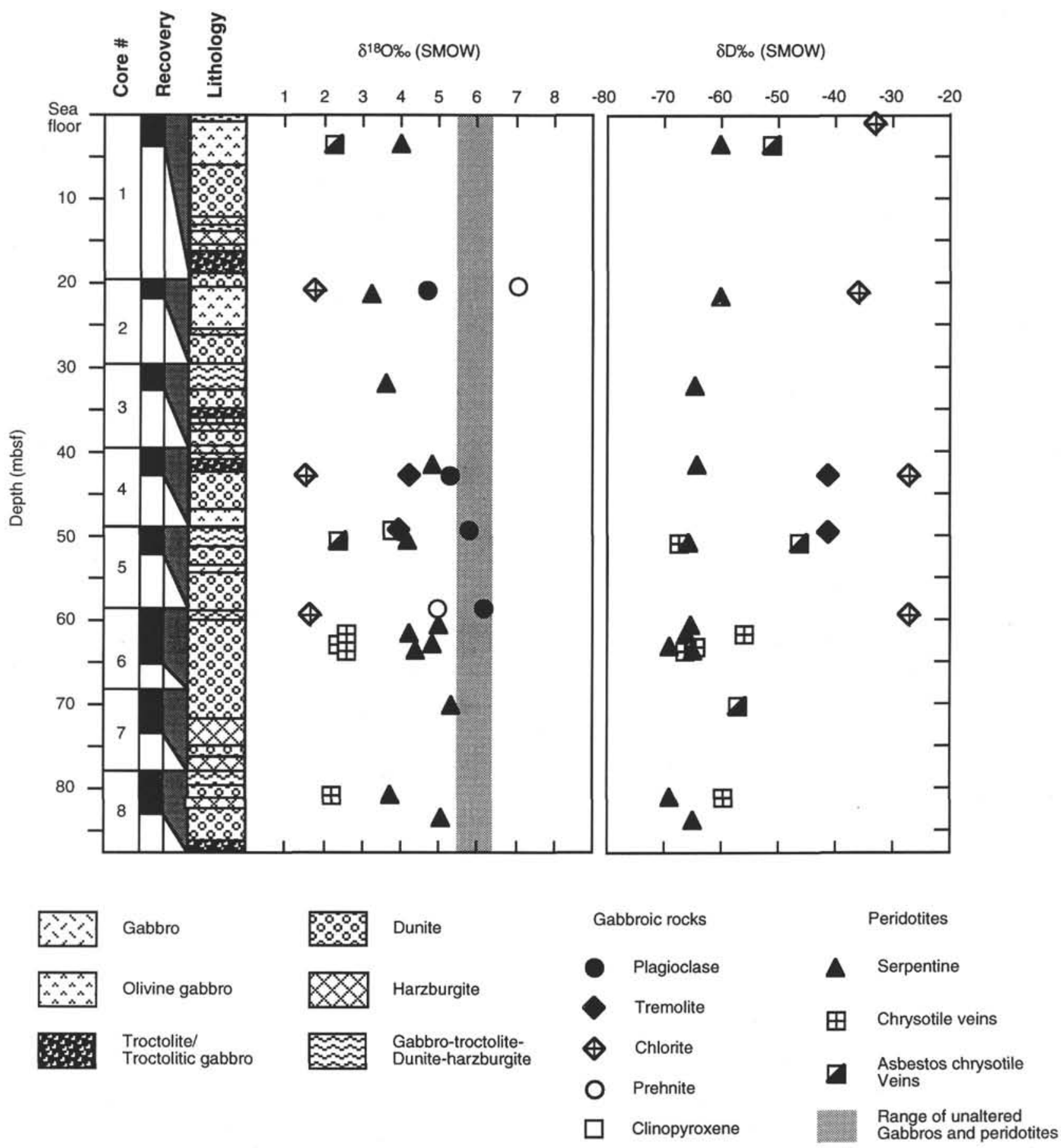

Figure 9. Variation in hydrogen and oxygen isotope ratios as a function of depth in Hole 895E.

and provide further constraints on the origin of the serpentinizing fluids. The amounts of $\mathrm{CO}_{2}$ produced during the experiments on dunite Sample 147-895E-2R-2 (Piece 9, 62-65 cm) were very reproducible with an average value of $100 \mu \mathrm{mol}$ of $\mathrm{CO}_{2}$ per gram of dunite (Fig. 10) and constant $\delta^{13} \mathrm{C}$ values of $-6 \%$. $\mathrm{CO}_{2}$ extracted from the harzburgite Sample 147-895E-8R-2 (Piece 7, 73-77 cm) also has low $\delta^{13} \mathrm{C}$ values of $-6 \%$ for temperatures of $600^{\circ}$ and $1000^{\circ} \mathrm{C}$. The two experiments performed at $800^{\circ} \mathrm{C}$ were slightly less reproducible, yielding $\delta^{13} \mathrm{C}$ values of $-7.8 \%$ and $-4.5 \%$ for identical amounts of $\mathrm{CO}_{2}$ (Fig. 10). The results of these experiments show that the amounts of $\mathrm{CO}_{2}$ are reproducible regardless of extraction temperature within a sample; but for the harzburgite sample, the amounts are significantly lower with an average value of $80 \mu \mathrm{mol}$ of $\mathrm{CO}_{2}$ per gram of rock. Carbonate was never observed in thin sections or by XRD of these two samples. The presence of trace carbonates could be a major source of $\mathrm{CO}_{2}$ during the heating of peridotites. However, similar experiments on the aragonite vein taken from Sample 147-895E-3R-2 (Piece 9, 96-98 cm), produced a low amount of $\mathrm{CO}_{2}(390 \mu \mathrm{mol})$ at $600^{\circ} \mathrm{C}$, with a $\delta^{13} \mathrm{C}$ value of $-1.7 \%$ (Fig. 10 ; Table 12 ). At $800^{\circ} \mathrm{C}$, decarbonation was quantitative; for a sample weight of $13.62 \mathrm{mg}$, we obtained $10,310 \mu \mathrm{mol}$ of $\mathrm{CO}_{2}$ and a characteristic marine $\delta^{13} \mathrm{C}$ value of $1 \%$ and a $\delta^{18} \mathrm{O}$ value of $33.2 \%$. The compositions obtained at 


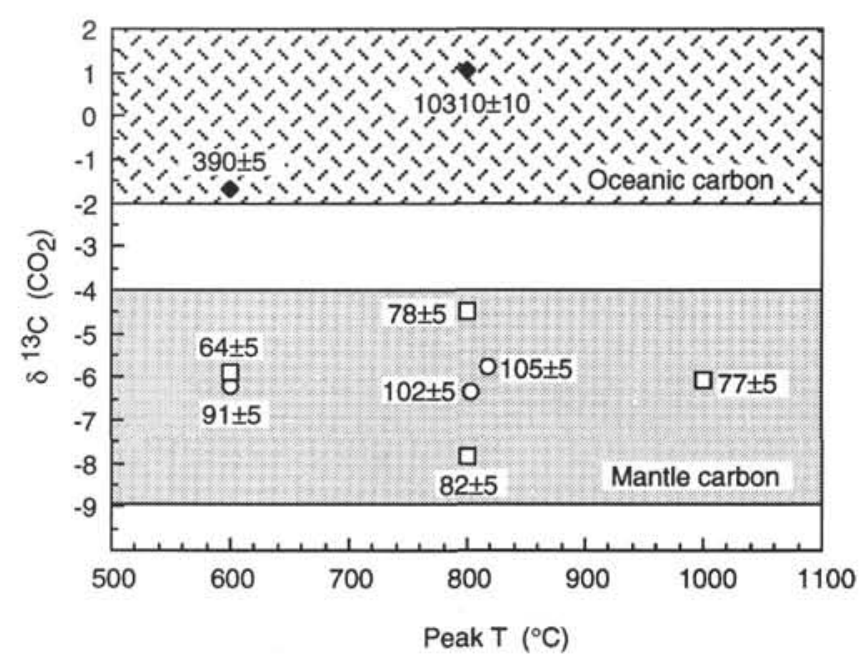

Figure $10 . \delta^{13} \mathrm{C}$ values of $\mathrm{CO}_{2}$ extracted from Hole $895 \mathrm{E}$ peridotites vs. peak temperatures recorded through the heating experiments. Numbers represent the $\mu \mathrm{mol}$ of $\mathrm{CO}_{2}$ per gram of rock. Open circle $=$ dunite $147-895 \mathrm{E}-2 \mathrm{R}-2,62$ $65 \mathrm{~cm}$; open square $=$ harzburgite $147-895 \mathrm{E}-8 \mathrm{R}-2,73-77 \mathrm{~cm}$; solid diamond $=$ aragonite vein from dunite $147-895 \mathrm{E}-3 \mathrm{R}-2,96-98 \mathrm{~cm}$. The range of carbon isotope ratios of mantle carbon (shaded region) is distinct from that of oceanic carbon (hatched region).

$800^{\circ} \mathrm{C}$ fall completely within the range of carbon and oxygen isotope ratios of the aragonite veins determined by conventional phosphoric acid techniques (Table 11), and indicate precipitation from unaltered seawater at ambient temperatures $\left(<10^{\circ} \mathrm{C}\right)$. The low $\delta^{13} \mathrm{C}$ value obtained at $600^{\circ} \mathrm{C}$ for the aragonite is the consequence of kinetically induced isotopic fractionation that accompanied the partial decarbonation of the aragonite sample.

The amounts of $\mathrm{CO}_{2}$ extracted from the Hess Deep peridotites are surprisingly high relative to amounts of $\mathrm{CO}_{2}$ that are preserved as vesicles trapped in marine basalts (Table 12; Pineau and Javoy, 1983; Mattey et al., 1984; Pineau and Javoy, 1994). Indeed, $\mathrm{CO}_{2}$ converted as carbon concentrations in Hole 895 peridotites range from 850 to $1200 \mathrm{ppm}$ of carbon in contrast to total carbon concentrations that do not exceed $200 \mathrm{ppm}$ for submarine basalts (Pineau and Javoy, 1994). These preliminary results suggest that deep oceanic rocks can trap larger amounts of carbon than shallow volcanics. We must emphasize that, to date, $\mathrm{CO}_{2}$ has only been analyzed in fresh mid-oceanridge basalt (MORB) glasses while our mantle peridotite samples are characterized by a high degree of serpentinization. A gabbro sample from Hole 894G (147-894G-9R-2, Piece 5B, 88-93 cm) also was heated to $1000^{\circ} \mathrm{C}$ under identical experimental conditions. The very small amount of liberated $\mathrm{CO}_{2}$ (less than $0.1 \mu \mathrm{mol}$ for a sample weight of $331 \mathrm{mg}$ ) implies that the large $\mathrm{CO}_{2}$ concentrations discussed previously are restricted to the shallow mantle peridotites.

\section{Troctolitic and Gabbroic Rocks}

Profiles of the hydrogen and oxygen isotope compositions of mineral separates and vein-fillings from the mafic rocks in Holes 895D and $895 \mathrm{E}$ are shown as a function of depth and lithology in Figures 8 and 9 . With the exception of one prehnite vein, all of the relict and secondary phases are depleted in ${ }^{18} \mathrm{O}$ relative to unaltered reference values. Oxygen isotope ratios of relict plagioclase are the most variable and range from $3.7 \%$ in the least altered olivine gabbro sample to $6.3 \%$ in highly altered, coarse-grained gabbros cut by discrete prehnite veins. Plagioclase in moderately altered samples with predominantly greenschist facies assemblages exhibit less variations and cluster about a value of $5 \pm 0.3 \%$ (Table 11). Tremolite forms two groups, with $\delta^{18} \mathrm{O}$ values of 5.1 in the olivine gabbros and values of

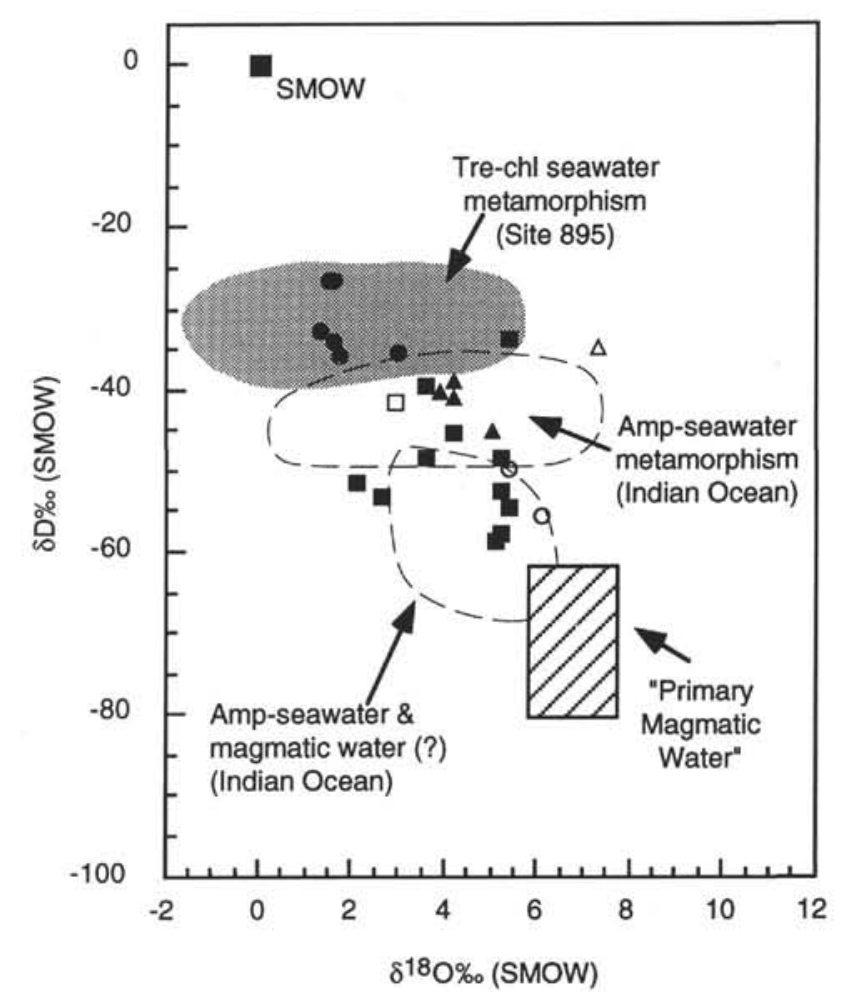

Figure 11. Hydrogen and oxygen isotope ratios of chlorite (solid circles) and tremolite (solid triangles) at Site 895 compared with isotopic compositions of (1) amphibole (solid squares), amphibole vein (open square), smectite veins (open triangles), and corrensite (open circles) from Site 894 (Früh-Green et al., this volume) and (2) equilibrium fields for hornblende during seawater metamorphism and seawater + magmatic water(?) metamorphism, defined by Stakes (1991) from Indian Ocean plutonic rocks. Shaded region is range of calculated isotopic compositions of chlorite and tremolite in equilibrium with modified seawater $\left(-1 \%_{0}\right.$ to $\left.4 \%{ }_{0} \delta^{18} \mathrm{O} ; 0 \% \%_{0} \pm 5 \%_{0} \delta \mathrm{D}\right)$ at temperatures of $350^{\circ}-600^{\circ} \mathrm{C}$ (see text for discussion and references).

approximately $4.3 \%$ in the coarse-grained diallage gabbros. The latter values are indistinguishable from the pyroxene compositions. Chlorites show the most negative $\delta^{18} \mathrm{O}$ values, ranging from $1.4 \%$ to $3.1 \%$ (Table 11 ).

A depletion in ${ }^{18} \mathrm{O}$ relative to unaltered oceanic crust is consistent with the serpentine data and clearly reflects exchange with hydrothermal fluids at temperatures greater than $350^{\circ}-400^{\circ} \mathrm{C}$ (Lécuyer et al., this volume; Früh-Green et al., this volume; Agrinier et al., in press). Since plagioclase is more sensitive to isotopic exchange than coexisting olivine or pyroxene, the variations in $\delta^{18} \mathrm{O}$ observed in plagioclase may record multiple phases of hydrothermal alteration over a large range in temperatures and varying fluid/rock ratios. The most depleted $\delta^{18} \mathrm{O}_{\text {plg }}$ value of 3.7 is interpreted as resulting from exchange with seawater at high temperatures. Although the more positive $\delta^{18} \mathrm{O}$ values in the highly altered gabbros are close to the range of primary magmatic plagioclase $(6.3 \pm 0.1 \%$; Dunn, 1986$)$, it is more likely that these values reflect progressive retrograde exchange at lower temperatures. Initiation of hydrothermal alteration at temperatures greater than $400^{\circ} \mathrm{C}$ is consistent with petrologic constraints on the greenschist-facies alteration and with results from the serpentinized peridotites discussed above and from gabbros at Site 894. Similar variations in plagioclase $\delta^{18} \mathrm{O}$ values in the Site 894 isotropic gabbros have been interpreted as reflecting high-temperature alteration $\left(450^{\circ}-\right.$ $600^{\circ} \mathrm{C}$ ) at low fluid/rock ratios, producing a general ${ }^{18} \mathrm{O}$-depletion, followed by varying degrees of retrograde exchange with lower temperature fluids, resulting in a shift back to positive values similar to 
Table 12. Amounts and $\delta^{13} \mathrm{C}$ values of $\mathrm{CO}_{2}$ extracted from Hole 895 peridotites by heating under vacuum.

\begin{tabular}{lrcc}
\hline \multicolumn{1}{c}{ Sample } & $\begin{array}{c}\text { Peak } \mathrm{T} \\
\left({ }^{\circ} \mathrm{C}\right)\end{array}$ & $\begin{array}{c}\mathrm{CO}_{2} \\
(\mu \text { mol/gm rock })\end{array}$ & $\begin{array}{c}\delta^{13} \mathrm{C} \\
(\mathrm{PDB})\end{array}$ \\
\hline Dunite & & & \\
147-895E-2R-2, 62-65 cm & 600 & 91 & -6.2 \\
& 800 & 102 & -6.3 \\
& 800 & 105 & -5.8 \\
Harzburgite & & & \\
147-895E-8R-2, 73-77 cm & 600 & 64 & -5.9 \\
& 800 & 82 & -7.8 \\
& 800 & 78 & -4.5 \\
Aragonite & 1000 & 77 & -6.1 \\
147-895E-3R-2, 96-98 cm & 600 & 392 & -1.7 \\
& 800 & 10,313 & 1.1 \\
\hline
\end{tabular}

Notes: Peak temperatures represent the maximum of temperature reached by the sample and maintained during 10 minutes.

or higher than the original magmatic signatures (Lécuyer et al., 1993; Lécuyer et al., this volume; Früh-Green et al., this volume). Variations in the bulk rock oxygen isotope ratios of altered gabbros from Hole $894 \mathrm{G}$ are largely a function of the modal abundance and isotopic composition of plagioclase (Lécuyer et al., 1993; Lécuyer et al., this volume). Retrograde isotopic overprinting under lower temperature conditions is consistent with petrographic observations, which indicate that the abundance of late alteration phases is largely related to deformation and the density of microfractures and discrete veins. Interestingly, the two oxygen isotope measurements of prehnite yielded relatively low $\delta^{18} \mathrm{O}$ values of $5.0 \%$ for prehnite alteration of plagioclase and $7.1 \%$ for vein prehnite. Like quartz, prehnite and zeolites tend to be enriched in ${ }^{18} \mathrm{O}$ and can exhibit values of $>10 \%$ in oceanic rocks even at moderate temperatures (e.g., Schiffman et al., 1984). These low ratios in prehnite are consistent with crystallization temperatures greater than $250^{\circ}-300^{\circ} \mathrm{C}$.

Clear isotopic disequilibrium characteristic of open system exchange is evident by the large variations in $\Delta_{\mathrm{plg} \text {-min }}$ (defined as $\delta^{18} \mathrm{O}_{\mathrm{plg}}$ $-\delta^{18} \mathrm{O}_{\text {mineral }}$ ) of the gabbro samples and largely reflects the varying degree of isotopic overprinting in plagioclase. For example, $\Delta_{\text {plg-cpx }} v a r-$ ies from 1 to $2 \%$, and are generally higher than values of $0.5-1.3 \%$ from plagioclase-clinopyroxene pairs from rapidly quenched, unaltered basalts or slowly cooled plutonic gabbros (Taylor, 1968). Although the plagioclase-chlorite fractionations vary considerably, the two least altered samples exhibit a constant fractionation of approximately $2.3 \%$ o. Assuming that this fractionation represents peak metamorphic re-equilibration and assuming that serpentine and chlorite are isotopically identical (Wenner and Taylor, 1971), approximate equilibrium temperatures of $400^{\circ}-450^{\circ} \mathrm{C}$ have been calculated by combining equation (2) above for the oxygen isotope fractionation between chlorite and water and the semi-empirical plagioclase-water fractionation curves of Bottinga and Javoy (1973). Interestingly, the fluids in equilibrium with plagioclase at these temperatures would have had an oxygen isotope composition of approximately $0.6 \%$ o$1 \%$, which is consistent with positive shifts in the oxygen isotope composition of hydrothermal fluids inferred from other studies of altered oceanic crust and ophiolites (e.g., Muehlenbachs and Clayton, 1976; Gregory and Taylor, 1981; Alt et al., 1986b; Kawahata et al., 1987; Muehlenbachs, 1987).

Hydrogen isotope ratios of chlorite lie in a narrow range between $-36 \%$ and $-27 \%$, with no clear distinction between the relatively fresh and highly altered and deformed samples. Tremolite is slightly more enriched in hydrogen, with $\delta \mathrm{D}$ values ranging from $-46 \%$ to $-39 \%$. Although the hydrogen isotope ratios of the serpentine in the surrounding ultramafic rocks at Hole $895 \mathrm{E}$ exhibit a slight trend towards more negative $\delta \mathrm{D}$ values (discussed above), no clear variation with depth is observed in the hydrous phases in the altered gabbros.
Experimental data on the $\mathrm{D} / \mathrm{H}$ fractionation between hydrous minerals and water is scarce and commonly contradictory; however, the $\delta \mathrm{D}$ values of the Site 895 chlorites are consistent with fractionations predicted by empirical and experimental $\mathrm{D} / \mathrm{H}$ fractionation curves for chlorite-water at temperatures greater than $350^{\circ}-400^{\circ} \mathrm{C}$ and assuming a seawater-dominated hydrothermal fluid (Wenner and Taylor, 1973; Graham et al., 1987), as shown in Figure 11. The D/H ratios of chlorite are similar to or slightly lower than values determined on bulk rock and chlorite mineral separates from greenschist-facies metamorphic basalts and sheeted dikes recovered at depths below 890 mbsf in Hole 504B (alteration zones III and IV; Kawahata et al., 1987). Hydrogen, strontium, and oxygen isotope studies from Hole $504 \mathrm{~B}$ indicate that alteration in rocks from the basalt-dike transition zone and sheeted dike sections took place at low fluid/rock ratios and temperatures up to $350^{\circ} \mathrm{C}$ in upflow regions of an axial hydrothermal system (Alt et al., 1986a; Kawahata et al., 1987).

In Figure 11, the hydrogen and oxygen isotope ratios of the hydrous minerals at Site 895 are compared with isotopic compositions of amphibole in Site 894 gabbros and in gabbros from the Indian Ocean (Stakes, 1991; Kempton et al., 1991; Früh-Green et al., this volume). The isotopic ratios of tremolite fall completely within the range of compositions of hornblende interpreted to have formed in equilibrium with seawater at temperatures of $400^{\circ}-500^{\circ} \mathrm{C}$ (Stakes, 1991), and to a large extent have $\mathrm{D} / \mathrm{H}$ ratios that are distinct from those in amphibole from Site 894 gabbros. However, one important complication to a simple scenario of seawater metamorphism arises from the dependence of isotopic fractionation on amphibole chemistry and from contradictions in hydrogen fractionation factors of tremolite-water. Experimental studies of Graham et al. (1984) indicate that the tremolite-water fractionation is relatively constant at $-22 \pm$ $2 \%$ over a temperature range of $350^{\circ}-650^{\circ} \mathrm{C}$ and is significantly different than the predicted fractionation curve using the octahedral cation model of Suzuoki and Epstein (1976). As the chemical composition of tremolite used in the experiments of Graham et al. (1984) is similar to the Site 895 tremolite compositions (in particular, with regard to $\mathrm{Na}(\mathrm{A})$ and $\mathrm{Al}^{\mathrm{V} 1}$ contents), we suggest that the calibration of Graham et al. is a better estimate of tremolite-water hydrogen isotope fractionation in our system. Thus, based on this assumption, the range of isotopic compositions of tremolite in equilibrium with seawater roughly overlaps with those of chlorite at temperatures of $350^{\circ}-$ $500^{\circ} \mathrm{C}$, as is indicated by the shaded region in Figure 11 . One important distinction becomes apparent: the isotopic signatures of chlorite suggest a seawater-dominant component of the hydrothermal fluid; whereas, the tremolite compositions are distinctly depleted in deuterium relative to equilibrium tremolite-seawater compositions. A deuterium depletion relative to seawater is consistent with the $\mathrm{D} / \mathrm{H}$ ratios observed in serpentine, and suggests that the isotopic composition of the hydrothermal fluids may not have been homogeneous over the entire alteration history. The relative depletion in deuterium in tremolite and serpentine at Site 895 , associated with ${ }^{18} \mathrm{O}$-enrichments in the serpentines, may reflect episodes in which the fluids were more enriched in a low- $\delta \mathrm{D} / \mathrm{high}^{-18} \mathrm{O}$ magmatic or metamorphic component. This is further consistent with carbon isotope ratios of $\mathrm{CO}_{2}$ extracted from the pervasively altered peridotites (Fig. 10).

\section{DISCUSSION}

The petrology and microfabrics of the shallow mantle sequences recovered at Site 895 indicate a complex, polyphase history of fluid infiltration and deformation under greenschist to zeolite facies conditions (Mével and Stamoudi, this volume). Metamorphism in the mafic and ultramafic rocks is dominated by hydration reactions and static replacement resulting in pseudomorphic replacement and coronitic textures. The overall degree of alteration and the distribution of lower temperature mineral assemblages is commonly related to an increase 
in cataclastic deformation and the density of microscopic and macroscopic veins (Kennedy et al., this volume). Cataclastic deformation tends to be more pronounced in the gabbroic rocks and is characterized by fracturing of fresh and pseudomorphed grains, incipient brecciation and the formation of multiple generations of veins filled with prehnite \pm hydrogrossular or zeolites.

\section{Conditions of Serpentinization}

Hydration reactions in the ultramafic lithologies are characterized by the formation of mesh-textured lizardite and magnetite \pm chlorite after olivine and bastite \pm chlorite \pm tremolite \pm talc after orthopyroxene, with lesser amounts of secondary diopside, brucite, clay, and rare antigorite. Serpentinization of anhydrous peridotite may be described by general reactions involving the magnesian end-member phases of reactants and products:

$$
\begin{aligned}
& 2 \mathrm{Mg}_{2} \mathrm{SiO}_{4}+3 \mathrm{H}_{2} \mathrm{O}=\mathrm{Mg}_{3} \mathrm{Si}_{2} \mathrm{O}_{5}(\mathrm{OH})_{4}+\mathrm{Mg}(\mathrm{OH})_{2} ; \\
& \text { Forsterite } \quad \mathrm{Mg} \text {-Serpentine } \mathrm{Mg} \text {-Brucite } \\
& 6 \mathrm{MgSiO}_{3}+3 \mathrm{H}_{2} \mathrm{O}=\mathrm{Mg}_{3} \mathrm{Si}_{2} \mathrm{O}_{5}(\mathrm{OH})_{4}+\mathrm{Mg}_{3} \mathrm{Si}_{4} \mathrm{O}_{10}(\mathrm{OH})_{2} ; \\
& \text { Enstatite } \quad \mathrm{Mg} \text {-Serpentine } \quad \mathrm{Mg} \text {-Talc }
\end{aligned}
$$

and

$$
\mathrm{Mg}_{2} \mathrm{SiO}_{4}+\mathrm{MgSiO}_{3}+2 \mathrm{H}_{2} \mathrm{O}=\mathrm{Mg}_{3} \mathrm{Si}_{2} \mathrm{O}_{5}(\mathrm{OH})_{4} \text {. }
$$

$$
\text { Forsterite Enstatite Mg-Serpentine }
$$

In the $\mathrm{MgO}-\mathrm{FeO}-\mathrm{SiOH}_{2} \mathrm{O}$ system, formation of magnetite accompanying serpentinization can be described by the generalized reaction:

Olivine + Enstatite $+\mathrm{H}_{2} \mathrm{O}=$ Serpentine + Magnetite $+\mathrm{H}_{2}$.

The temperature range at which serpentinization occurs in the oceanic crust has been the subject of considerable debate. Experimental and phase-equilibrium studies indicate that serpentine-forming reactions can occur over a wide range of temperatures up to approximately $500^{\circ} \mathrm{C}$, but depend greatly on the attainment of equilibrium and the presence or absence of excess $\mathrm{H}_{2} \mathrm{O}$ (e.g., Bowen and Tuttle, 1949; Johannes, 1968; and reviews in Moody, 1976, 1979; Elthon, 1981; Janecky and Seyfried, 1986; Chernosky et al., 1988; and O'Hanley et al., 1989). Estimates of peak metamorphic conditions prove difficult because equilibrium conditions may rarely be attained in oceanic peridotites, and multiple phases of serpentinization and varying $\mathrm{P}_{\mathrm{H} 2 \mathrm{O}}$ are common. Furthermore, the stability of lizardite, the dominant serpentine group mineral in oceanic serpentinites, is highly dependent on $\mathrm{Al}$ content (see review in Chernosky et al., 1988); and chrysotile and lizardite may persist metastably well into the antigorite stability field. Recent experiments by Janecky and Seyfried (1986) indicate that reactions (4) through (7) to produce the assemblage serpentine \pm brucite \pm talc proceed spontaneously at $300^{\circ} \mathrm{C}$ ( 500 bars), with variable intermediate assemblages and reaction paths. Although talc may be produced during serpentinization by reaction (5), it also forms together with olivine and vapor \pm chlorite during breakdown reactions of lizardite at temperatures greater than approximately $450^{\circ} \mathrm{C}$ in regionally metamorphic serpentinites (Evans and Trommsdorff, 1970; Trommsdorff and Evans, 1972, 1974; Caruso and Chernosky, 1979; Chernosky et al., 1988; Evans and Guggenheim, 1988). The experiments of Janecky and Seyfried (1986) have shown that formation of talc at temperatures below $300^{\circ} \mathrm{C}$ is limited and is controlled by fluid composition and high activities of $\mathrm{SiO}_{2}, \mathrm{MgO}$, and $\mathrm{H}^{+}$. The formation of tremolite is also associated with the breakdown of serpentine and diopside and can be described by the general reaction:

$$
\begin{array}{ccc}
\mathrm{Mg}_{3} \mathrm{Si}_{2} \mathrm{O}_{5}(\mathrm{OH})_{4}+8 \mathrm{CaMgSi}_{2} \mathrm{O}_{6}=4 \mathrm{Ca}_{2} \mathrm{Mg}_{5} \mathrm{Si}_{8} \mathrm{O}_{22}(\mathrm{OH})_{2} \\
\text { Serpentine } & \text { Diopside } & \text { Tremolite } \\
+8 \mathrm{Mg}_{2} \mathrm{SiO}_{4}+27 \mathrm{H}_{2} \mathrm{O} &
\end{array}
$$

Forsterite

This reaction is characteristic of medium-grade regional metamorphism of ultramafic rocks (Trommsdorff and Evans, 1972, 1974; Evans, 1977) and has been calibrated at $415^{\circ} \mathrm{C}$ and $2 \mathrm{~kb} \mathrm{P}_{\mathrm{H} 2 \mathrm{O}}$ (Evans and Trommsdorff, 1970; Peacock, 1987). The assemblage chlorite + tremolite \pm talc is favored by calcium-bearing fluids and high water/ rock ratios (Elthon, 1981).

Serpentine + talc \pm magnetite assemblages in the Hess Deep peridotites tend to be restricted to the presence of orthopyroxene, and is more common in the harzburgites. Alteration to serpentine + brucite \pm magnetite occurs in both dunite and harzburgite and is associated with local greater modal abundance of olivine relative to pyroxene (Hemley et al., 1977; Janecky and Seyfried, 1986). Tremolite and chlorite occur as replacement products of clino- and orthopyroxene; whereby chlorite is also a common vein-filling phase and an abundance of tremolite is associated with late aragonite veins. This general association of primary minerals and metamorphic assemblages implies that the distribution and composition of secondary phases in the Site 895 peridotites depends greatly on (1) primary mineral modes, (2) local gradients in the activity $\mathrm{MgO}$ and $\mathrm{SiO}_{2}$ to produce talc and $\mathrm{Al}_{2} \mathrm{O}_{3}$ and $\mathrm{CaO}$ to produce tremolite and chlorite, and (3) fluid chemistries during veining. Local equilibrium conditions limited to a grain-size scale is indicated by the presence of tremolite and brucite, which is typically unstable under geologically relevant P-T conditions and should react to form diopside and chrysotile (Evans and Trommsdorff, 1970; Evans, 1977). Calcium produced during hydration of pyroxene was at least partly consumed by tremolite forming reactions and may largely reflect the original $\mathrm{Ca}$ content of the anhydrous protolith (Bonatti et al., 1984; Komor et al., 1985; Hébert et al., 1990). This may have important consequences when considering mass balance relationships between serpentinization in the peridotites and incipient rodingitization in the gabbroic rocks at Hess Deep (Mével and Stamoudi, this volume). Although the assemblage chlorite + tremolite \pm talc could provide evidence for early serpentine-dehydration reactions, unequivocal evidence for the formation of olivine, for example, as dictated by reaction (8), has not be found in the samples that we examined. However, by combining experimental and field data of serpentinized peridotites, a maximum temperature of approximately $450^{\circ} \mathrm{C}$ with low $\mathrm{X}_{\mathrm{CO}}$, of the fluid is indicated by the assemblage lizardite \pm antigorite, chlorite, tremolite, and talc in the Site 895 harzburgites. It may be surprising that at these temperatures, the thermodynamically stable serpentine-group mineral, antigorite, is not more abundant in the Hess Deep peridotites. Antigorite in oceanic peridotites is relatively rare and is commonly restricted to local shear zones (e.g., Prichard, 1979, 1980; Moody, 1979; Elthon, 1981; Hébert et al., 1990). The low abundance of antigorite in the Hess Deep peridotites and in other oceanic serpentinites does not preclude higher peak metamorphic temperatures, but may reflect a number of factors, such as: low pressures; the low nucleation and reaction rates of antigorite; the expanded stability of Al-rich lizardite; and the metastable growth and persistence of lizardite at higher temperatures (Moody, 1979; Elthon, 1981; Chernosky et al., 1988).

Progressive serpentinization of the ultramafic rocks is associated with multiple generations of macroscopic veins filled with serpentine \pm brucite \pm chlorite \pm talc \pm magnetite. This suggests that hydration of the peridotites was enhanced by multiple phases of fracturing and interaction with water-rich fluids during successive pulses of hydro- 
thermal activity. Enrichments in chlorine in brucite/serpentine mixed phases in the dunites may indicate local infiltration of alkaline, $\mathrm{MgCl}_{2}$-bearing solutions (Janecky and Seyfried, 1986). Channelled fluid flow continued under zeolite facies conditions, as indicated by the presence of serpentine \pm magnetite \pm clay \pm zeolite veins that cross the pervasive serpentine-brucite veins in dunites in Hole $895 \mathrm{E}$. The latest veining event is associated with the influx of oxidizing, $\mathrm{CO}_{2}-$ and Ca-rich fluids, depositing aragonite in discrete fractures and microfractures and producing incipient brecciation of the serpentinized wall rock. The formation of tremolite in discontinuous veinlets, patches, and as pseudomorphs of pyroxene locally accompanies the aragonite veins. Stable isotope analyses of aragonite indicate crystallization at ambient temperatures in the presence of pure seawater (Table 11; Bonatti et al., 1980).

Serpentinization over a temperature range of $350^{\circ}-450^{\circ} \mathrm{C}$ is consistent with the relatively constant and low $\delta^{18} \mathrm{O}$ values of serpentine separates and vein serpentines in the ultramafic rocks (Figs. 7 through 9; Table 11). A depletion in ${ }^{18} \mathrm{O}$ relative to mantle reference values is characteristic of hydrothermal alteration of the lower oceanic crust at temperatures greater than $300^{\circ}$ (Gregory and Taylor, 1981; Cocker et al., 1982; Alt et al., 1986a; Muehlenbachs, 1987). Although oxygen isotope fractionation factors for serpentine are poorly known, the serpentine-magnetite oxygen isotope fractionations provide rough constraints on the conditions of serpentinization and indicate relatively high temperatures of $400^{\circ} \mathrm{C} \pm 50^{\circ}$.

The carbon isotope compositions of $\mathrm{CO}_{2}$ extracted from Hess Deep peridotites (Fig. 10) suggest a mantle origin for the carbon when we compare the range of $\delta^{13} \mathrm{C}$ values $(-7.8$ to $-4.5 \%$ ) found in our samples with the data obtained either from fresh MORBs of the Mid-Atlantic Ridge ( $-9 \%$ to $-4 \%$; Pineau and Javoy, 1983) or from different oceanic environments ( -7.5 to $-4.2 \%$; Mattey et al., 1984). The average $\delta^{13} \mathrm{C}$ value of $-6 \%$ for Hess Deep samples is also in good agreement with the estimate of the mantle carbon value of $-5.5 \%$ based on mass balance considerations between the different terrestrial carbon reservoirs (Hoefs, 1982).

Other origins for the $\mathrm{CO}_{2}$ trapped in Hess Deep peridotites can be considered, but are not supported by our data for the following reasons. A contribution of carbon derived from marine carbonates will produce $\delta^{13} \mathrm{C}$ values close to $0 \%$ as shown by $\mathrm{CO}_{2}$ produced by the decarbonation of aragonite veins (Fig. 10). Moreover, in the range of experimental temperatures $\left(600^{\circ}-1000^{\circ} \mathrm{C}\right)$, strong variations in the amounts of $\mathrm{CO}_{2}$ would be expected to accompany incomplete decarbonation processes at $600^{\circ} \mathrm{C}$ (Fig. 10), as seen in the experiments of the aragonite vein. $\mathrm{CO}_{2}$ could also result from oxidation by $\mathrm{H}_{2} \mathrm{O}$ of a reduced form of carbon (for example, as $\mathrm{CH}_{4}$ ) during the heating procedure, but the $\delta^{13} \mathrm{C}$ values should be lower, in the range of $-20 \%$ to $-35 \%$ o (Hoefs, 1982). Thus, the analyzed $\mathrm{CO}_{2}$ is more compatible with a magmatic origin, occurring as $\mathrm{CO}_{2}$ trapped in metamorphic minerals such as serpentine or chlorite. It remains to determine how this $\mathrm{CO}_{2}$ is trapped, either in inclusions or as substitution for other species such as $\mathrm{H}_{2} \mathrm{O}$ in hydrous minerals. It is unlikely that $\mathrm{CO}_{2}$ trapped in inclusions within magmatic minerals such as olivine or pyroxenes could survive the metamorphic reactions and pervasive serpentinization. We, therefore propose that $\mathrm{CO}_{2}$ is trapped during the serpentinization of the peridotites. This interpretation is consistent with the oxygen and hydrogen ratios in the Site 895 serpentines, and further supports the hypothesis of a contribution of magmatic volatiles, at least episodically, to the seawater-dominated hydrothermal fluids responsible for serpentinization. The hydrogen isotope ratios of serpentines in the Hess Deep peridotites, together with mass balance considerations, suggest that $\mathrm{H}_{2}$ produced during serpentinization reactions was a further component of the hydrothermal fluids. Therefore, volatiles released during hydration of peridotite at lower levels than those drilled during Leg 147 as well as during magma-degassing and melt-rock interactions associated with the emplacement of mafic melts in the peridotites at Site 895, may have played a critical role in the serpentinization of the shallow mantle at Hess Deep.

\section{Metamorphism in the Mafic Rocks and Conditions of Incipient Rodingitization}

Early static metamorphism in the troctolitic and gabbroic rocks at Site 895 is characterized by greenschist facies assemblages of tremolite, chlorite, secondary diopsidic clinopyroxene, and secondary anorthitic plagioclase, with rare talc and epidote (Mével and Stamoudi, this volume). Hydration reactions involved olivine and plagioclase to produce the distinct coronas of chlorite and diopsidic clinopyroxene, as well as the pseudomorphic replacement of olivine by tremolite and chlorite \pm talc and anorthitic plagioclase \pm chlorite \pm tremolite after plagioclase. Zoned $\mathrm{Al}$ concentrations in the tremolite and chlorite as well as a dependence of chlorite composition on precursor mineralogies suggest that these reactions involved local equilibrium and were controlled by diffusion of $\mathrm{Mg}$ from olivine and $\mathrm{Al}$ and $\mathrm{Ca}$ from plagioclase. The relatively high $\mathrm{Al}$ and $\mathrm{Mg}$ contents and the nearly linear relationship between the Tschermak substitution and $\mathrm{X}_{\mathrm{Mg}}$ observed in the chlorites (Fig. 6B) may to a large extent reflect these general disequilibrium conditions in a non-buffered system rather than indicate progressive equilibrium crystallization from epidote amphibolite to greenschist facies conditions in a buffered chemical system.

Progressive phases of fluid infiltration and overprinting to zeolite facies conditions is related to an increase in brittle deformation and resulted in the formation of hydrous $\mathrm{Ca}-\mathrm{Al}$ silicate and $\mathrm{Al}$-rich phases characteristic of incipient rodingitization. Hydration reactions produced prehnite, tremolite, chlorite, hydrogrossular, thomsonite, kaolinite, and boehmite after plagioclase, and tremolite, secondary diopside, chlorite, mixed layered chlorite/smectite ( \pm serpentine), and iron hydroxides after olivine and/or primary pyroxene. Lower temperature alteration clearly coincides with an increase in the density of microfractures and veins, and it is associated with incipient brecciation of the plagioclase grains and brittle fracturing of coarse-grained clinopyroxene. The extremely low Fe contents in the prehnite and the relative paucity of epidote in the Hess Deep mafic rocks suggests that the hydrothermal fluids had low $\mathrm{fO}_{2}$ (Liou et al., 1983; Rose and Bird, 1987).

The temperature and pressure conditions during the alteration history of the Site 895 troctolitic and gabbroic rocks are difficult to estimate due to a lack of characteristic mineral paragenesis and the overall tendency towards chemical disequilibrium. However, general constraints on the physical conditions of metamorphism can be made by comparing phase equilibria in the end-member $\mathrm{CaO}-\mathrm{MgO}-\mathrm{Al}_{2} \mathrm{O}_{3}$ $\mathrm{SiO}_{2}-\mathrm{H}_{2} \mathrm{O}$ system, taking into account Tschermak solution in chlorite (Fig. 12). Equilibrium thermodynamic calculations indicate that the reaction anorthite + forsterite $+\mathrm{H}_{2} \mathrm{O}=$ tremolite + diopside + chlorite occurs between $640^{\circ}-700^{\circ} \mathrm{C}$ at pressures of $1-2.5 \mathrm{~kb}$ (Connolly, 1990). The composition of chlorite at these conditions is $75 \%$ clinochlore and $25 \%$ amesite (chl75). The equilibrium assemblage diopside + anorthite + clinochlore (chl75) $+\mathrm{H}_{2} \mathrm{O}=$ tremolite + clinozoisite has an upper stability limit of approximately $430^{\circ}-540^{\circ} \mathrm{C}$ at pressures of 0.5-5 kb (Fig. 12; Connolly, 1990; Frey et al., 1991) and provides a minimum temperature estimate for the early hydrothermal alteration in the Site 895 gabbros. This range in temperatures is consistent with oxygen isotope fractionation temperatures for plagioclase-chlorite pairs (discussed above) and the temperature constraints deduced for the formation of antigorite, lizardite, chlorite, tremolite and talc in the ultramafic rocks. Experimental and thermodynamic data indicate that prehnite has a stability range of approximately $220^{\circ}-420^{\circ} \mathrm{C}$ (Liou et al., 1983; Frey et al., 1991; Connolly and Kerrick, 1985) and essentially overlaps the (poorly constrained) stability field of hydrogrossular (Yoder, 1950; Pistorius and Kennedy, 1960; Coleman, 1967). Phase equilibria in the pure $\mathrm{CaO}-\mathrm{MgO}-\mathrm{Al}_{2} \mathrm{O}_{3}-\mathrm{SiO}_{2}-$ $\mathrm{H}_{2} \mathrm{O}$ system predicts that prehnite is stable at low pressures below the invariant point for quartz-clinozoisite-garnet-prehnite- $\mathrm{H}_{2} \mathrm{O}$ at 1.35 $\mathrm{kb}, 420^{\circ} \mathrm{C}$, and that prehnite-forming reactions occur at distinctly lower temperatures than for tremolite-diopside-chlorite assemblages (Fig. 12; Connolly, 1990). The minor modal abundancies of charac- 


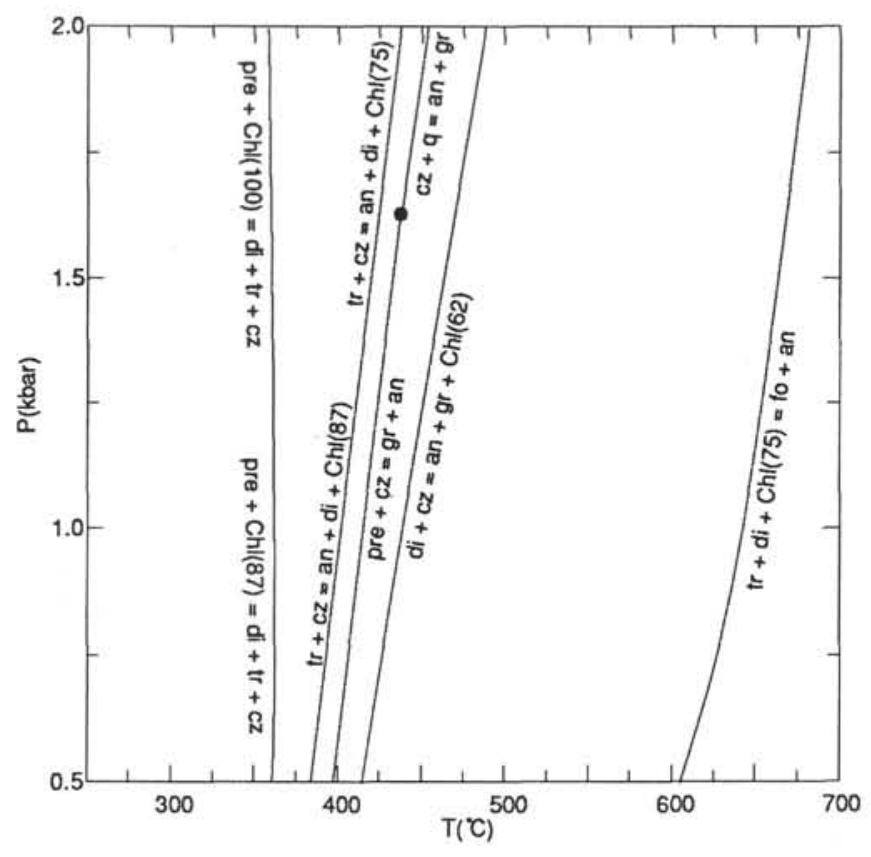

Figure 12. Calculated phase equilibria in the end-member $\mathrm{CaO}-\mathrm{MgO}-\mathrm{Al}_{2} \mathrm{O}_{3}$ $\mathrm{SiO}_{2}-\mathrm{H}_{2} \mathrm{O}$ system, taking into account Tschermak solution in chlorite, using the program PERPLEX (Connolly, 1990). Reaction equations are written such that the high-temperature assemblage is on the right of the $=$ sign. Abbreviations: $\mathrm{an}=$ anorthite $; \mathrm{chl}=$ chlorite solid solution, with the number in parentheses giving the modal percent of clinochlore; $\mathrm{cz}=$ clinozoisite; $\mathrm{di}=$ diopside; fo = forsterite; $\mathrm{gr}=$ grossular; $\mathrm{pre}=$ prehnite; $\mathrm{q}=$ quartz; $\mathrm{tr}=$ tremolite.

teristic low-temperature chlorite/smectite clays and the relatively low $\delta^{18} \mathrm{O}$ values of plagioclase in the highly altered gabbros suggests that the majority of the hydrothermal alteration took place at temperatures above approximately $200^{\circ}-250^{\circ} \mathrm{C}$. Microfabrics suggest that multiple phases of fluid-infiltration under greenschist to zeolite facies conditions were pre-, syn- and post-kinematic.

The close association between calc-silicate bearing mafic rocks and serpentinized peridotites has commonly been interpreted as a result of metasomatic processes involving the mobilization of $\mathrm{Ca}$-rich fluids released by serpentinization of ultramafic rocks (e.g., Honnorez and Kirst, 1975; Bideau et al., 1991; Hekinian et al., 1993). Although there is no doubt that the two processes are related, the crystallization of calc-silicate phases during hydrothermal alteration of the Hess Deep shallow mantle sequences may not merely be the result of infiltration of Ca-rich fluids generated during serpentinization. A predominance of $\mathrm{Ca}$-rich phases, as well as the $\mathrm{Mg}$-rich compositions of early chlorite, could also reflect (1) an original, relatively Caand $\mathrm{Mg}$-rich /Si-poor bulk composition of the mafic rocks and/or (2) circulation of evolved hydrothermal, high $\mathrm{pH}$ aqueous fluids enriched in calcium (and possibly Al?) during alteration of the overlying basaltic dikes and high level gabbros (Evans, 1977; Mottl and Holland, 1978; Mottl, 1983; Alt et al, 1986a; Seyfried, 1987; Seyfried et al., 1988). Calcium released during serpentinization may have played a negligible role in the process of incipient rodingitization at Hess Deep, due to the fact that the primary peridotite compositions are relatively clinopyroxene-poor (and thus Ca-poor). Furthermore, microtextural relationships suggest that the original Ca was bound in tremolite and secondary diopsidic pyroxene in the peridotites during the early phase of hydrous alteration of the ultramafic rocks.
The stable isotope data from the shallow mantle sequences at Site 895 suggests that the isotopic composition of the hydrothermal fluids may not have been homogeneous over the entire alteration history and that contributions of magmatic and/or metamorphic volatiles to the hydrothermal fluids may have been important. The presence of discontinuous diopside veinlets in plagioclase and the coronitic nature of chlorite and diopsidic clinopyroxene, in contrast to the static radiating replacement habit of tremolite after olivine in the gabbroic rocks could reflect slight differences in timing and mechanism of the early phases of alteration (Pl. 2, Figs. 1 through 3 ). The difference in hydrogen isotope ratios of chlorite and tremolite, and their inferred constraints on the $\mathrm{D} / \mathrm{H}$ ratios of the hydrothermal fluids, implies that seawater penetrated into the shallow mantle sequence at an early stage in the metamorphic history, and that later pulses of hydrother$\mathrm{mal}$ activity were influenced by processes of magma-degassing and/ or hydrogen production during serpentinization. The presence of $\mathrm{CO}_{2}$ with carbon of mantle origin in the Hess Deep serpentinites is strong evidence for the existence of a magmatic component in the hydrothermal fluids circulating in the deep layers of the oceanic crust.

The metamorphic mineral assemblages and vein types in the peridotites and gabbroic rocks at Site 895 are similar to those dredged from the ultrafast Garret Transform that intersects the East Pacific Rise near $13^{\circ} 25^{\prime} \mathrm{S}$ (Bideau et al., 1991). Both areas show a close association between processes of serpentinization and the formation of $\mathrm{Ca}$-rich assemblages in troctolitic and olivine-bearing gabbroic rocks under greenschist facies to zeolite facies conditions. The background and vein mineral parageneses observed in the Site 895 peridotites are also broadly similar to those reported from ODP Site 670, on the west flank of the slow-spreading Mid-Atlantic Ridge Rift Valley (Hébert et al., 1990). However, the Hole 670A serpentinites are interpreted as a diapiric intrusion of upper mantle rocks (Hébert et al., 1990); whereas at Hess Deep, the relatively low degree of deformation in the serpentinized peridotites and the static nature of alteration in the ultramafic and mafic rocks suggest that processes of crustal attenuation and faulting associated with the formation of the rift valley were the dominant mechanisms of uplift and emplacement of deep crustal rocks at shallow levels in the oceanic crust (compare Francheteau et al., 1990). Extension and faulting would have enhanced permeabilities and allowed the penetration of seawater and/or evolved hydrothermal fluids, possibly mixed with volatile components released during magma degassing, producing the static metamorphic and vein assemblages observed in the Site 895 shallow mantle sequences. Together with mass balance considerations, the trend towards higher $\delta^{18} \mathrm{O}$ values and lower $\delta \mathrm{D}$ values observed in Hole $895 \mathrm{E}$ (Fig. 9) may provide evidence for a flux not only of mantle-derived volatiles from the mantle but also $\mathrm{H}_{2}\left( \pm \mathrm{CH}_{4}\right)$ released during serpentinization of the underlying peridotite. An extension model for the formation of the Hess Deep Rift Valley is further supported by restored orientations of veins and magmatic foliations (MacLeod et al., 1993; MacLeod et al., this volume) and by paleomagnetic measurements in Hole $895 \mathrm{E}$, which indicate relatively consistent tectonic rotations of $\approx 36^{\circ}$ subsequent to the major serpentinization event that produced the magnetite that contributes to the bulk magnetization of the rocks (Gillis, Mével, Allan, et al., 1993; Shipboard Scientific Party, 1993a, 1993b; Kelso et al., this volume).

\section{SUMMARY AND CONCLUSIONS}

The harzburgites, dunites, and associated troctolitic and gabbroic rocks recovered at the Hess Deep Valley represent shallow mantle sequences created at the East Pacific Rise. These rocks record a polyphase history of hydrothermal alteration and provide constraints on the depth and mechanisms of hydrothermal circulation. The complex sequence of harzburgite-dunite-troctolite-gabbro at Site 895 is considered to be the result of multiple generations of partial melting 
and competing processes of melt migration, wall-rock reaction and cumulus olivine precipitation close to the mantle/crust boundary (Gillis, Mével, Allan, et al., 1993; Dick et al., 1993; Mével et al., 1993; Arai and Matsukage, this volume; Allan and Dick, this volume; Dick and Natland, this volume; Kennedy et al., this volume). Petrologic and stable isotope data as well as microtextural relationships suggest that the initiation of static metamorphism, dominated by hydration reactions in the peridotites and gabbroic rocks, occurred at temperatures of approximately $450^{\circ}-550^{\circ} \mathrm{C}$, and may indicate that uplift and penetration of seawater-dominated fluids occurred at an early stage during the propagation of the Cocos-Nazca rift. Progressive serpentinization of the ultramafic rocks is associated with multiple generations of macroscopic veins filled with serpentine \pm brucite \pm chlorite \pm talc \pm magnetite. This suggests that hydration of the peridotites was enhanced by multiple phases of fracturing and interaction with water-rich fluids during successive pulses of hydrothermal activity. Progressive hydrothermal alteration from greenschist to zeolite facies and incipient rodingitization of the gabbroic rocks is indicated by tremolite + chlorite + secondary diopside + secondary anorthitic plagioclase \pm talc \pm prehnite \pm epidote assemblages in the least altered samples, with increasing proportions of prehnite, hydrogrossular, zeolites, clays, and $\mathrm{Al}$ - and $\mathrm{Fe}$-hydroxides as cataclastic deformation and veining increases.

Oxygen isotope ratios of mineral separates from the gabbros and peridotites from Site 895 show a depletion in $\delta^{18} \mathrm{O}$ relative to mantle values and are consistent with high-temperature exchange with aqueous fluids. $\mathrm{D} / \mathrm{H}$ ratios of chlorite, serpentine, and tremolite ratios, together with $\delta^{13} \mathrm{C}$ values of $\mathrm{CO}_{2}$ extracted from completely serpentinized dunites, suggest at least two, but possibly three, components of the hydrothermal fluids: hydrothermally altered seawater; magmatic volatiles; and $\mathrm{H}_{2}$ released during serpentinization. These data combined with structural data suggest that penetration of seawater and high temperatures $\left(>350^{\circ} \mathrm{C}\right)$ hydrothermal alteration produced a low${ }^{18} \mathrm{O}$ shallow mantle sequence at some distance off-axis of the EPR, but at an early stage in the propagation of the Cocos-Nazca rift and formation of Hess Deep. The predominance of $\mathrm{Ca}-\mathrm{Al}$ silicate assemblages in the gabbroic rocks may be controlled by primary bulk compositions or the compositions of evolved hydrothermal fluids, rather than solely reflecting the generation and mobilization of Ca-rich fluids during serpentinization processes. The relatively low degree of deformation in the serpentinized peridotites and the static nature of alteration in the ultramafic and mafic rocks suggest that processes of crustal attenuation and faulting associated with the formation of the rift valley were the dominant mechanisms of uplift and emplacement of deep crustal rocks at shallow levels in the oceanic crust (compare Francheteau et al., 1990).

The results of this study suggest that seawater-peridotite interactions and high-temperature serpentinization processes may be an important contribution to the overall ${ }^{18} \mathrm{O}$-budget in the oceanic lithosphere, producing ${ }^{18} \mathrm{O}$-depleted sequences which balance the ${ }^{18} \mathrm{O}$-enrichments during low temperature alteration of the upper oceanic crust. This process may play a more significant role in buffering the oxygen isotope composition of seawater than has been considered previously. Our study also suggests that serpentinized peridotites may be an important sink for mantle-derived $\mathrm{CO}_{2}$ and a significant source of $\mathrm{H}_{2}$ (in addition to $\mathrm{CH}_{4}$ ) in hydrothermal systems. Furthermore, previous studies at Hess Deep, as well as the overall results of Leg 147 provide evidence that serpentinization processes may be important in the formation of marine magnetic anomalies (Gillis, Mével, Allan, et al., 1993; Shipboard Scientific Party, 1993a, 1993b; Richter et al., 1993; Kelso et al., this volume).

\section{ACKNOWLEDGMENTS}

This study was supported by ETH grant No. 0-20-710-93. We would like to thank E. Reusser and P. Ulmer for assistance with mi- croprobe analyses and interpretation of the data, J. Connolly for calculating the phase equilibria and for many helpful discussions, and D. Pozzorini for running the lab while G.F-G. was at sea. The help of A. Lamair and T. Gianettoni in sample preparation and mineral separation was invaluable for the completion of this project. Assistance of Z. Sharp during laser microanalytical extractions is gratefully acknowledged. G.F-G. and C.L. would particularly like to acknowledge the enormous efforts of the Co-Chief Scientists, K. Gillis and C. Mével, during and after the cruise, and would like to thank the staff and crew of the JOIDES Resolution for their perseverance, hard work, and friendliness during the tri-holiday cruise. We would also like to thank P. J. Saccocia and D. A. O'Hanley for their constructive and helpful reviews of our rather long manuscript.

\section{REFERENCES}

Abrajano, T.A., Sturchio, N.C., Bohlke, J.K., Lyon, G.L., Poreda, R.J., and Stevens, C.M., 1988. Methane-hydrogen gas seeps, Zambales Ophiolite, Philippines: deep or shallow origin? Chem. Geol., 71:211-222.

Agrinier, P., Hekinian, R., Bideau, D., and Javoy, M., in press. Stable isotope compositions $\left({ }^{18} \mathrm{O} /{ }^{16} \mathrm{O}, \mathrm{D} / \mathrm{H}\right.$, and $\left.{ }^{13} \mathrm{C} /{ }^{12} \mathrm{C}\right)$ of oceanic crust and upper mantle rocks exposed in the Hess Deep near the Galapagos Triple Junction. Earth Planet. Sci. Lett.

Agrinier, P., Javoy, M., and Girardeau, J., 1988. Hydrothermal activity in a peculiar oceanic ridge: oxygen and hydrogen isotope evidence in the $\mathrm{Xi}$ gaze ophiolite (Tibet, China). Chem. Geol., 71:313-335.

Allan, J.F., and Leg 147 Scientific Party, 1993. Leg 147 Cr-spinel: petrogenetic inferences regarding melt impregnation of Hess Deep peridotites. Eos, 74 (Suppl.):661.

Alt, J.C., Honnorez, J., Laverne, C., and Emmermann, R., 1986a. Hydrothermal alteration of a $1 \mathrm{~km}$ section through the upper oceanic crust, Deep Sea Drilling Project Hole 504B: mineralogy, chemistry, and evolution of seawater-basalt interactions. J. Geophys. Res., 91:10309-10335.

Alt, J.C., Muehlenbachs, K., and Honnorez, J., 1986b. An oxygen isotopic profile through the upper kilometer of the oceanic crust, DSDP Hole 504B. Earth Planet. Sci. Lett., 80:217-229.

Bideau, D., Hebert, R., Hekinian, R., and Cannat, M., 1991. Metamorphism of deep-seated rocks from the Garret Ultrafast Transform (East Pacific Rise near $13^{\circ} 25^{\prime}$ S). J. Geophys. Res., 96:10079-10099.

Boillot, G., Grimaud, S., Mauffret, A., Mougenot, D., Kornprobst, J., Mergoil-Daniel, J., and Torrent, G., 1980. Ocean-continent boundary off the Iberian margin: a serpentinite diapir west of the Galicia Bank. Earth Planet. Sci. Lett., 48:23-34.

Bonatti, E., 1976. Serpentinite protrusions in the oceanic crust. Earth Planet. Sci. Lett., 32:107-113.

Bonatti, E., Lawrence, J.R., Hamlyn, P.R., and Breger, D., 1980. Aragonite from deep sea ultramafic rocks. Geochim. Cosmochim. Acta, 44:12071214.

Bonatti, E., Lawrence, J.R., and Morandi, N., 1984. Serpentinization of ocean-floor peridotites. Earth Planet. Sci. Lett., 70:88-94.

Bonatti, E., and Michael, P.J., 1989. Mantle peridotites from continental rifts to ocean basins to subduction zones. Earth Planet. Sci. Lett., 91:297311.

Bonatti, E., Seyler, M., Channell, J., Giraudeau, J., and Mascle, G., 1990. Peridotites drilled from the Tyrrhenian Sea, ODP Leg 107. In Kastens, K.A., Mascle, J., et al., Proc. ODP, Sci. Results, 107: College Station, TX (Ocean Drilling Program), 37-47.

Borthwick, J., and Harmon, R.S., 1982. A note regarding $\mathrm{CIF}_{3}$ as an alternative to $\mathrm{BrF}_{5}$ for oxygen isotope analysis. Geochim. Cosmochim. Acta, 46:1665-1668.

Bottinga, Y., and Javoy, M., 1973. Comments on oxygen isotope geothermometry. Earth Planet. Sci. Lett., 20:250-265.

Bougault, H., Charlou, J.L., Fouquet, Y., Needham, H.D., Vaslet, N., Appriou, P., Baptiste, P.J., Rona, P.A., Dmitriev, L., and Silantiev, S., 1993. Fast and slow spreading ridges: structure and hydrothermal activity, ultramafic topographic highs, and $\mathrm{CH}_{4}$ output. J. Geophys. Res., 98:96439651 .

Bowen, N.L., and Tuttle, O.F., 1949. The system MgO-SiO $\mathrm{O}_{2}-\mathrm{H}_{2} \mathrm{O}$. Geol. Soc. Am. Bull., 60:439-460.

Burkhard, D.J., and O'Neil, J.R., 1988. Contrasting serpentinization processes in the eastern Central Alps. Contrib. Mineral. Petrol., 99:498-506.

Caruso, L.G., and Chernosky, J.V., Jr., 1979. The stability of lizardite. Can. Mineral., 17:757-769. 
Charlou, J.L., Bougault, H., Appriou, P., Jean-Baptiste, P., Etoubleau, J., and Birolleau, A., 1991a. Water column anomalies associated with hydrothermal activity between $11^{\circ} 40^{\prime}$ and $13^{\circ} \mathrm{N}$ on the East Pacific Rise: discrepancies between tracers. Deep-Sea Res., 38:569-596.

Charlou, J.L., Bougault, H., Appriou, P., Nelsen, T., and Rona, P., 1991 b. Different TDM/ $\mathrm{CH}_{4}$ hydrothermal plume signatures: TAG site at $26^{\circ} \mathrm{N}$ and serpentinized ultrabasic diapir at $15^{\circ} 05^{\prime} \mathrm{N}$ on the Mid-Atlantic Ridge. Geochim. Cosmochim. Acta, 55:3209-3222.

Charlou, J.L., and Donval, J.-P., 1993. Hydrothermal methane venting between $12^{\circ} \mathrm{N}$ and $26^{\circ} \mathrm{N}$ along the mid-Atlantic ridge. J. Geophys. Res. 98:9625--9642.

Chernosky, J.V., Jr., Berman, R.G., and Bryndzia, L.T., 1988. Stability, phase relations, and thermodynamic properties of chlorite and serpentine group minerals. Rev. Mineral., 19:295-346.

Clayton, R.N., O'Neil, J., and Mayeda, T.K., 1972. Oxygen isotope exchange between quartz and water. J. Geophys. Res., 77:3057-3067.

Cocker, J.D., Griffin, B.J., and Muehlenbachs, K., 1982. Oxygen and carbon isotope evidence for seawater-hydrothermal alteration of the Macquarie Island ophiolite. Earth Planet. Sci. Lett., 61:112-122.

Coleman, R.G., 1967. Low-temperature reaction zones and alpine ultramafic rocks of California, Oregon and Washington. U.S. Geol. Surv. Bull., 1247:49.

Connolly, J.A.D., 1990. Calculation of multivariable phase diagrams: an algorithm based on generalized thermodynamics. Am. J. Sci., 290:666718

Connolly, J.A.D., and Kerrick, D.M., 1985. Experimental and thermodynamic data on the stability of prehnite. Eos, 66:388. (Abstract)

Dick, H.J.B., and Bullen, T., 1984. Chromian spinel as a petrogenetic indicator in abyssal and alpine-type peridotites and spatially associated lavas. Contrib. Mineral. Petrol., 86:54-76.

Dick, H.J.B., Natland, J., and Leg 147 Scientific Party, 1993. Site 895 sheeted dunites and melt transport in the shallow mantle beneath the East Pacific Rise. Eos, 74 (Suppl.):661.

Dunn, T., 1986. An investigation of the oxygen isotope geochemistry of the Stillwater Complex. J. Petrol., 27:987-997.

Elthon, D., 1981. Metamorphism in oceanic spreading centers. In Emiliani, C. (Ed.), The Sea (Vol. 7): The Oceanic Lithosphere; New York (Wiley), 285-303.

Evans, B.W., 1977. Metamorphism of alpine peridotite and serpentinite. Annu. Rev, Earth Planet. Sci., 5:398-447.

Evans, B.W., and Guggenheim, S., 1988. Talc, pyrophyllite, and related minerals. Rev. Mineral., 19:225-294.

Evans, B.W., and Trommsdorff, V., 1970. Regional metamorphism of ultramafic rocks in the Central Alps: parageneses in the system $\mathrm{CaO}-\mathrm{MgO}$ $\mathrm{SiO}_{2}-\mathrm{H}_{2}$ O. Schweiz. Mineral. Petrogr. Mitt., 50:481-492.

Francheteau, J., Armijo, R., Cheminée, J.L., Hekinian, R., Lonsdale, P.F., and Blum, N., 1990. 1 Ma East Pacific Rise oceanic crust and uppermost mantle exposed by rifting in Hess Deep (equatorial Pacific Ocean). Earth Planet. Sci. Lett., 101:281-295.

, 1992. Dyke complex of the East Pacific Rise exposed in the walls of Hess Deep and the structure of the upper oceanic crust. Earth Planet. Sci. Lett., 111:109-121.

Freund, F., Wengeler, H., and Martens, R., 1982. A deuterium-hydrogen fractionation mechanism in magnesium oxide. Geochim. Cosmochim. Acta, 46:1821-1829.

Frey, M., de Capitani, C., and Liou, J.G., 1991. A new petrogenetic grid for low-grade metabasites. J. Metamorph. Geol., 9:497-509.

Friedman, I., and O'Neil, J.R., 1977. Compilation of stable isotope fractionation factors of geochemical interest. In Fleischer, M. (Ed.), Data of Geochemistry (6th ed.). Geol. Surv. Prof. Pap. U.S., 440-KK:1-12.

Frost, B.R., 1985. On the stability of sulfides, oxides, and native metals in serpentine. J. Petrol., 26:31-63.

Früh-Green, G.L., Plas, A., Grobéty, B., and Lécuyer, C., 1995. Multi-stage hydrothermal alteration and antigorite serpentenisation of EPR shallow mantle at Hess Deep. Terra Abstr., 7:208.

Früh-Green, G.L., Weissert, H., and Bernoulli, D., 1990. A multiple fluid history recorded in Alpine ophiolites. J. Geol. Soc. London, 147:959970 .

Gillis, K., Mével, C., Allan, J., et al., 1993, Proc, ODP, Init. Repts., 147: College Station, TX (Ocean Drilling Program).

Graham, C.M., Harmon, R.S., and Sheppard, S.M.F., 1984. Experimental hydrogen isotope studies: hydrogen isotope exchange between amphibole and water. Am. Mineral., 69:128-138.
Graham, C.M., Viglino, J.A., and Harmon, R.S., 1987. Experimental study of hydrogen-isotope exchange between aluminous chlorite and water and of hydrogen diffusion in chlorite. Am. Mineral., 72:566-579.

Gregory, R.T., and Taylor, H.P., Jr., 1981. An oxygen isotope profile in a section of Cretaceous oceanic crust, Samail ophiolite, Oman: evidence for $\delta^{18} \mathrm{O}$-buffering of the oceans by deep ( $>5 \mathrm{~km}$ ) seawater-hydrothermal circulation at mid-ocean ridges. J. Geophys. Res., 86:2737-2755.

Hammarstrom, J.M., and Zen, E.-A., 1986. Aluminium in hornblende: an empirical igneous geobarometer. Am. Mineral., 71:1297-1313.

Heaton, T.H.E., and Sheppard, S.M.F., 1977. Oxygen and hydrogen isotopic evidence for sea-water hydrothermal alteration and ore deposition, Troodos complex, Cyprus. In Volcanic Processes in Ore Genesis, Inst. Min. Metall., Geol. Soc. Spec. Publ., 7:42-57.

Hébert, R., Adamson, A.C., and Komor, S.C., 1990. Metamorphic petrology of ODP Leg 109, Hole 670A serpentinized peridotites: serpentinization processes at a slow spreading ridge environment. In Detrick, R., Honnorez, J., Bryan, W.B., Juteau, T., et al., Proc. ODP, Sci. Results, 106/ 109: College Station, TX (Ocean Drilling Program), 103-115.

Hekinian, R., Bideau, D., Francheteau, J., Cheminée, J.L., Armijo, R., Lonsdale, P., and Blum, N., 1993. Petrology of the East Pacific Rise crust and upper mantle exposed in the Hess Deep (eastern equatorial Pacific). $J$. Geophys. Res., 98:8069-8094.

Hemley, J.J., Montoya, J.W., Christ, C.L., and Hostetler, P.B., 1977. Mineral equilibria in the $\mathrm{MgO}-\mathrm{SiO}_{2}-\mathrm{H}_{2} \mathrm{O}$ system: talc-chrysotile-forsterite-brucite stability relations. Am. J. Sci., 277:322-351.

Hey, M.H., 1954. A new review of the chlorites. Mineral. Mag., 30:227-292.

Hoefs, J., 1982. Isotope geochemistry of carbon. In Schmidt, H.-L., Förstel, H., and Heinzinger, K. (Eds.), Stable Isotopes: Amsterdam (Elsevier), $103-113$.

Honnorez, J., and Kirst, P., 1975. Petrology of rodingites from the Equatorial Mid-Atlantic fracture zones and their geotectonic significance. Contrib. Mineral. Petrol., 49:233-257.

Ito, E., and Clayton, R.N., 1983. Submarine metamorphism of gabbros from the Mid-Cayman Rise: an oxygen isotopic study. Geochim. Cosmochim. Acta, 47:535-546.

Janecky, D.R., and Seyfried, W.E., Jr., 1986. Hydrothermal serpentinization of peridotite within the oceanic crust: experimental investigations of mineralogy and major element chemistry. Geochim. Cosmochim. Acta, 50:1357-1378.

Johannes, W.. 1968. Experimental investigation of the reaction forsterite + $\mathrm{H}_{2} \mathrm{O}=$ serpentine + brucite. Contrib. Mineral. Petrol., 19:309-315.

Karson, J.A., Hurst, S.D., and Lonsdale, P.F., 1992. Tectonic rotations of dikes in fast-spread oceanic crust exposed near Hess Deep. Geology, 20:685-688.

Karson, J.A., Thompson, G., Humphris, S.E., Edmond, J.M., Bryan, W.B., Brown, J.R., Winters, A.T., Pockalny, R.A., Casey, J.F., Campbell, A.C., Klinkhammer, G., Palmer, M.R., Kinzler, R.J., and Sulanowska, M.M., 1987. Along-axis variations in seafloor spreading in the MARK area. $\mathrm{Na}$ ture, 328:681-685.

Kawahata, H., Kusakabe, M., and Kikuchi, Y., 1987. Strontium, oxygen and hydrogen isotope geochemistry of hydrothermally altered and weathered rocks in DSDP Hole 504B, Costa Rica Rift. Earth Planet. Sci. Lett., $85: 343-355$

Kelley, D.S., 1994. The influence of spreading rate on fluid evolution in submarine magma-hydrothermal systems. Eos, 75:649.

Kelley, D.S., and Früh-Green, G.L., 1995. Methane concentrations and isotopic compositions in layer 3 of the oceanic crust. Eos, 76:44.

Kelley, D.S., Hoering, T., and Frantz, J.D., 1994. Methane-rich fluids in gabbroic rocks from the Southwest Indian Ridge: results from mass spectrometric analyses. Eos, 44:657.

Kempton, P.D., Hawkesworth, C.J., and Fowler, M., 1991. Geochemistry and isotopic composition of gabbros from layer 3 of the Indian Ocean crust, Hole 735B. In Von Herzen, R.P., Robinson, P.T., et al., Proc. ODP, Sci. Results, 118: College Station, TX (Ocean Drilling Program), 127-143.

Kennedy, L.A., Boudier, F., Kronenberg, A.K., and the Leg 147 Scientific Party, 1993. Deformation and recovery in the upper mantle and microstructural constraints on melt impregnations. Eos, 74:661.

Komor, S.C., Elthon, D.E., and Casey, J.F., 1985. Serpentinization of cumulate ultramafic rocks from the North Arm Mountain massif of the Bay of Islands ophiolite. Geochim. Cosmochim. Acta, 49:2331-2338.

Kyser, T.K., 1987. Stable isotope variations in the mantle. Rev. Mineral., $16: 141-164$. 
Kyser, T.K., and Kerrich, R., 1991. Retrograde exchange of hydrogen isotopes between hydrous minerals and water at low temperatures. In Taylor, H.P., Jr., O'Neil, J.R., and Kaplan, I.R. (Eds.), Stable Isotope Geochemistry: A Tribute to Samuel Epstein, Spec. Publ. Geochem. Soc., 3:409-422.

Laird, J., 1988. Chlorites: metamorphic petrology. Rev. Mineral., 19:405447.

Lécuyer, C., and Fourcade, S., 1991. Oxygen isotope evidence for multistage hydrothermal alteration at a fossil slow-spreading center: the Silurian Trinity Ophiolite (California, U.S.A.). Chem. Geol. (Isot. Geosci. Sect.), 87:231-246.

Lécuyer, C., Gruau, G., Reynard, B., Mével, C., and the Leg 147 Scientific Party, 1993. High-temperature diffusion of seawater through gabbroic rocks at Hess Deep Rift Valley, Leg 147. Eos, 74:653. (Abstract)

Liou, J.G., Kim, H.S., and Maruyama, S., 1983. Prehnite-epidote equilibria and their petrologic applications. J. Petrol., 24:321-342.

Lonsdale, P., 1988. Structural pattern of the Galapagos microplate and evolution of the Galapagos triple junctions. J. Geophys. Res., 93:1355113574.

MacLeod, C.J., Boudier, F., Célérier, B., Kennedy, L.A., and Leg 147 Scientific Party, 1993. Structure of the lower oceanic crust in Hess Deep from ODP Leg 147 cores and borehole electrical images. Eos, 74:653-654.

Maruyama, S., Suzuki, K., and Liou, J.G., 1983. Greenschist-amphibolite transition equilibria at low pressures. J. Petrol., 24:583-604.

Mattey, D.P., Carr, R.H., Wright, I.P., and Pillinger, C.T., 1984. Carbon isotopes in submarine basalts. Earth Planet. Sci. Lett., 70:196-206.

McCrea, J.M., 1950. On the isotopic chemistry of carbonates and a paleotemperature scale. J. Chem. Phys., 18:849-857.

Mellini, M., Trommsdorff, V., and Compagnoni, R., 1987. Antigorite polysomatism: behavior during progressive metamorphism. Contrib. Mineral. Petrol., 97:147-155.

Mével, C., Cannat, M., and Leg 147 Scientific Party, 1993. Two types of magmatic intrusions in oceanic peridotites. Eos, 74:661.

Moody, J.B., 1976. Sepentinization: a review. Lithos, 9:125-138.

1979. Serpentines, spilites and ophiolite metamorphism. Can. Mineral., 17:871-887.

Mottl, M.J., 1983. Metabasalts, axial hot sprigs, and the structure of hydrothermal systems at mid-ocean ridges. Geol. Soc. Am. Bull., 94:161-180.

Mottl, M.J., and Holland, H.D., 1978. Chemical exchange during hydrothermal alteration of basalt by seawater, I. Experimental results for major and minor components of seawater. Geochim. Cosmochim. Acta, 42:11031115.

Muehlenbachs, K., 1987. Alteration of the oceanic crust and the ${ }^{18} \mathrm{O}$ history of seawater. Rev. Mineral., 16:425-444.

Muehlenbachs, K., and Clayton, R.N., 1976. Oxygen isotope composition of the oceanic crust and its bearing on seawater. J. Geophys. Res., 81:43654369.

O'Hanley, D.S., Chernosky, J.V., Jr., and Wicks, F.J., 1989. The stability of lizardite and chrysotile. Can. Mineral., 27:483-493.

O'Neil, J.R., and Taylor, H.P., Jr., 1969. Oxygen isotope fractionation between muscovite and water. J. Geophys. Res., 74:6012-6022.

Peacock, S.M., 1987. Serpentinization and infiltration metasomatism in the Trinity peridotite, Klamath province, northern California: implications for subduction zones. Contrib. Mineral. Petrol., 95:55-70.

Pineau, F., and Javoy, M., 1983. Carbon isotopes and concentrations in midoceanic ridge basalts. Earth Planet. Sci. Lett., 62:239-257.

, 1994. Strong degassing at ridge crests: the behaviour of dissolved carbon and water in basalt glasses at $14^{\circ} \mathrm{N}$, Mid-Atlantic Ridge. Earth Planet. Sci. Lett., 123:179-198.

Pistorius, C.W.S.T., and Kennedy, G.C., 1960. Stability relations of grossularite and hydrogrossularite at high temperatures and pressures. Am. J. Sci., 258:247-257.

Prichard, H.M., 1979. A petrographic study of the process of serpentinization in ophiolites and the ocean crust. Contrib. Mineral. Petrol., 68:231-241.

1980. The petrology and genesis of oceanic serpentines and gabbros [Ph.D. thesis]. Univ. Newcastle-Upon-Tyne.

Richter, C., Kelso, P., Pariso, J.E., and Leg 147 Shipboard Party, 1993. Magnetic properties and anisotropies of upper mantle and lower crustal rocks (ODP Sites 894 and 895). Eos, 74:660.

Rona, P., Bougault, H., Charlou, J.L., Appriou, P., Nelsen, T., Tefry, T.H., Eberhart, G.L., Barone, A., and Needham, H.D., 1992. Hydrothermal cir- culation, serpentinization, and degassing at a rift valley-fracture zone intersection: Mid-Atlantic Ridge near $15^{\circ} \mathrm{N}, 45^{\circ} \mathrm{W}$. Geology, 20:783-786.

Rose, N.M., and Bird, D.K., 1987. Prehnite-epidote phase relations in the Nordre Aputiteq and Kruuse Fjord layered gabbros. J. Petrol., 28:11931218.

Sakai, H., and Tsutsumi, M., 1978. D/H fractionation factors between serpentine and water at $100^{\circ} \mathrm{C}$ to $500^{\circ} \mathrm{C}$ and 2000 bar pressure, and the $\mathrm{D} / \mathrm{H}$ ratios of natural serpentines. Earth Planet. Sci. Lett., 40:231-242.

Sakai, R., Kusakabe, M., Noto, M., and Ishii, T., 1990. Origin of waters responsible for serpentinization of the Izu-Ogasawara-Mariana forearc seamounts in view of hydrogen and oxygen isotope ratios. Earth Planet. Sci. Lett., 100:291-303.

Schiffman, P., Williams, A.E., and Evarts, R.C., 1984. Oxygen isotope evidence for submarine hydrothermal alteration of the Del Puerto ophiolite, California. Earth Planet. Sci. Lett., 70:207-220.

Seyfried, W.E., and Dibble, W.E., 1980. Seawater-peridotite interaction at $300^{\circ} \mathrm{C}$ and 500 bars: implications for the origin of oceanic serpentinites. Geochim. Cosmochim. Acta, 44:309-321.

Seyfried, W.E., Jr., 1987. Experimental and theoretical constraints on hydrothermal alteration processes at mid-ocean ridges. Annu. Rev. Earth Planet. Sci., 15:317-335.

Seyfried, W.E., Jr., Berndt, M.E., and Seewald, J.S., 1988. Hydrothermal alteration processes at mid-ocean ridges: constraints from diabase alteration experiments, hot-spring fluids and composition of the oceanic crust. Can. Mineral., 26:787-804.

Sharp, Z., and Kirschner, D., 1994. Quartz-calcite oxygen isotope thermometry: a calibration based on natural isotopic variations. Geochim. Cosmochim. Acta, 58:4491-4501.

Sharp, Z.D., 1990. A laser-based microanalytical method for the in situ determination of oxygen isotope ratios of silicates and oxides. Geochim. Cosmochim. Acta, 54:1353-1357.

Sheppard, S.M.F., 1980. Isotopic evidence for the origins of water during metamorphic processes in oceanic crust and ophiolite complexes. Colloq. Int. C.N.R.S., 272:135-147.

Shipboard Scientific Party [ODP Leg 147], 1993a. Offset drilling pierces the Hess Deep Rift Valley. Geotimes, 93:19-21.

1993b. Pacific lower crustal and shallow mantle sections recovered. Eos, 74:297, 307.

Stakes, D.S., 1991. Oxygen and hydrogen isotope compositions of oceanic plutonic rocks: high-temperature deformation and metamorphism of oceanic layer 3. In Taylor, H.P., Jr., O'Neil, J.R., and Kaplan, I.R. (Eds.), Stable Isotope Geochemistry: A Tribute to Samuel Epstein. Spec. Publ. Geochem. Soc., 3:77-90.

Stakes, D.S., and Taylor, H.P., Jr., 1992. The northern Samail Ophiolite: an oxygen isotope, microprobe, and field study. J. Geophys. Res., 97:70437080 .

Suzuoki, T., and Epstein, S., 1976. Hydrogen isotope fractionation between $\mathrm{OH}$-bearing minerals and water. Geochim. Cosmochim. Acta, 40:12291240.

Taylor, H.P., Jr., 1968. The oxygen isotope geochemistry of igneous rocks. Contrib. Mineral. Petrol., 19:1-71.

1977. Water/rock interactions and the origin of $\mathrm{H}_{2} \mathrm{O}$ in granitic batholiths. J. Geol. Soc. London, 133:509-558.

, 1984. Oxygen and hydrogen isotope studies of hydrothermal interactions at submarine and subaerial spreading centers. In Rona, P.A., Boström, K., Laubier, L., and Smith, K.L., Jr. (Eds.), Hydrothermal Processes at Seafloor Spreading Centers: New York (Plenum Press), 83139.

Thompson, G., 1983. Basalt-seawater interaction. In Rona, P.A., Boström, K., Laubier, L., and Smith, K.L., Jr. (Eds.), Hydrothermal Processes at Seafloor Spreading Centers: New York (Plenum), 225-278.

Trommsdorff, V., and Evans, B.W., 1972. Progressive metamorphism of antigorite schist in the Bergell tonalite aureole (Italy). Am. J. Sci., 272:423437.

, 1974. Alpine metamorphism of peridotitic rocks. Schweiz. Mineral. Petrogr. Mitt., 54:33-352.

1977. Antigorite-ophicarbonates: contact metamorphism in Valmalenco, Italy. Contrib. Mineral. Petrol., 62:301-312.

Von Damm, K.L., Edmond, J.M., Grant, B., Measures, C.I., Walden, B., and Weiss, R.F., 1985. Chemistry of submarine hydrothermal solutions at $21^{\circ} \mathrm{N}$, East Pacific Rise. Geochim. Cosmochim. Acta, 49:2197-2220. 
Wenner, D.B., and Taylor, H.P., 1971. Temperatures of serpentinization of ultramafic rocks based on $\mathrm{O}^{18} / \mathrm{O}^{16}$ fractionation between coexisting serpentine and magnetite. Contrib. Mineral. Petrol., 32:165-185.

- 1973. Oxygen and hydrogen isotope studies of the serpentinization of ultramafic rocks in oceanic environments and continental ophiolitic complexes. Am. J. Sci., 273:202-239.

1974. $\mathrm{D} / \mathrm{H}$ and $\mathrm{O}^{18} / \mathrm{O}^{16}$ studies of serpentinization of ultramafic rocks. Geochim. Cosmochim. Acta, 38:1255-1286.

Whittaker, E.J.W., and Zussman, J., 1956. The characterization of serpentine minerals by X-ray diffraction. Mineral. Mag., 31:107-125.
Wicks, F.J., and O'Hanley, D.S., 1988. Serpentine minerals: structure and petrology. Rev. Mineral., 19:91-101.

Wicks, F.J., and Plant, A.G., 1979. Electron microprobe and X-ray microbeam studies of serpentine minerals. Can. Mineral., 17:785-830.

Yoder, H.S., 1950. Stability relations of grossularite. J. Geol., 58:221-253.

Date of initial receipt: 3 August 1994

Date of acceptance: 27 April 1995

Ms 147SR-016 

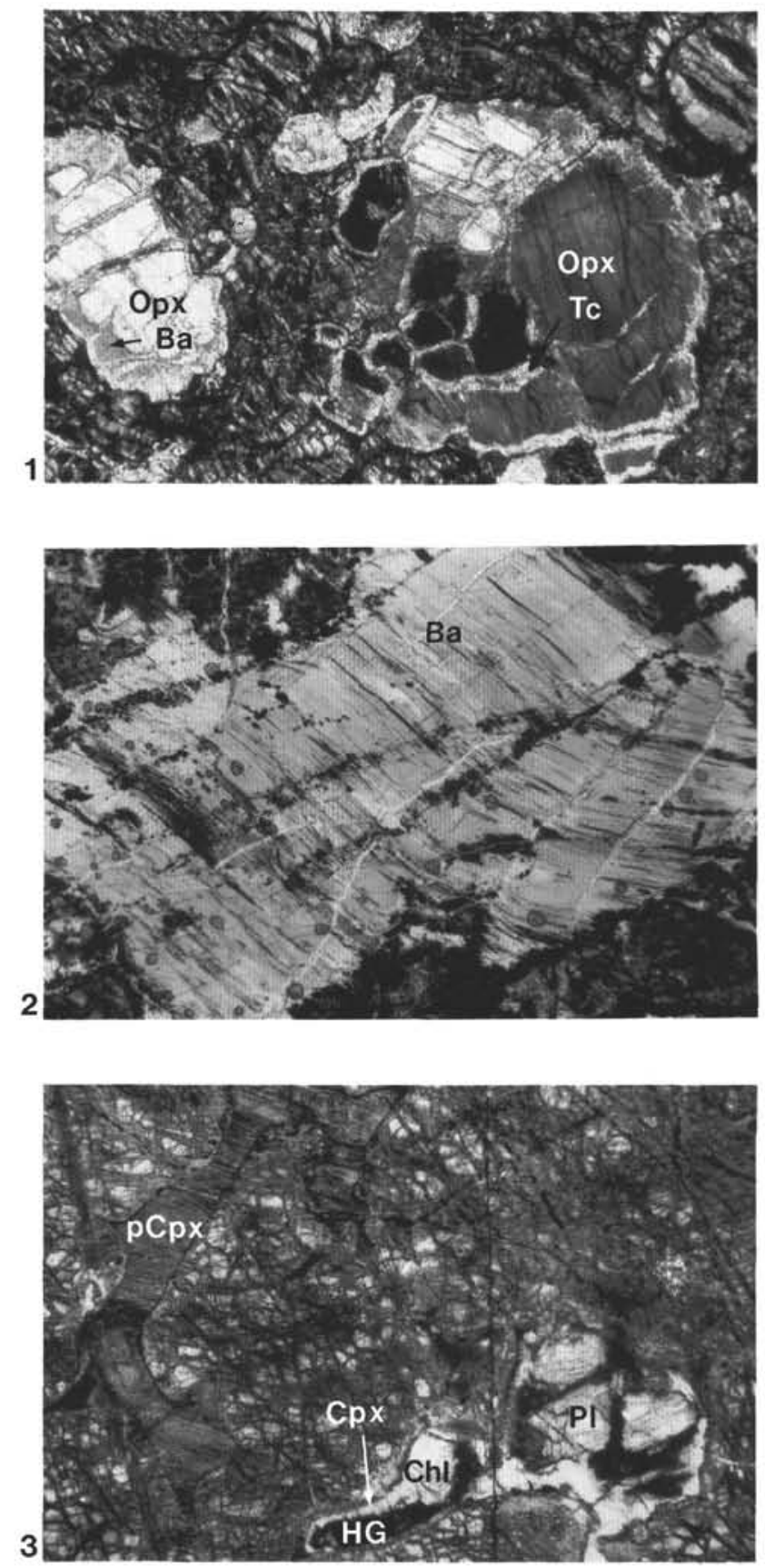

Plate 1. Photomicrographs of primary mineral alteration in Site 895 ultramafic rocks. The width of all photographs represents $3.6 \mathrm{~mm}$. 1. Harzburgite Sample 147-895E-1R-3 (Piece 3B, 71-73 cm). Relatively fresh orthopyroxene grains (Opx) in pervasively serpentinized olivine groundmass. Alteration of orthopyroxene consists of thin talc rims and fracture fillings (Tc) and less developed bastite replacement (Ba). 2. Harzburgite Sample 147-895D-4R-4 (Piece 14, 96-99 $\mathrm{cm}$ ). Pseudomorphic serpentine replacement after primary orthopyroxene. The bastite (Ba) is kinked. 3. Impregnated dunite Sample 147-895C-4R-3 (Piece 2A, $13-16 \mathrm{~cm})$. Plagioclase $(\mathrm{Pl})$ alteration phases and coarse-grained poikilitic clinopyroxene $(\mathrm{pCpx})$ in serpentine-olivine groundmass. Irregular hydrogrossular (HG) and chlorite $(\mathrm{Chl})$ replacements after plagioclase are outlined by a thin primary clinopyroxene $(\mathrm{Cpx})$ rim. 

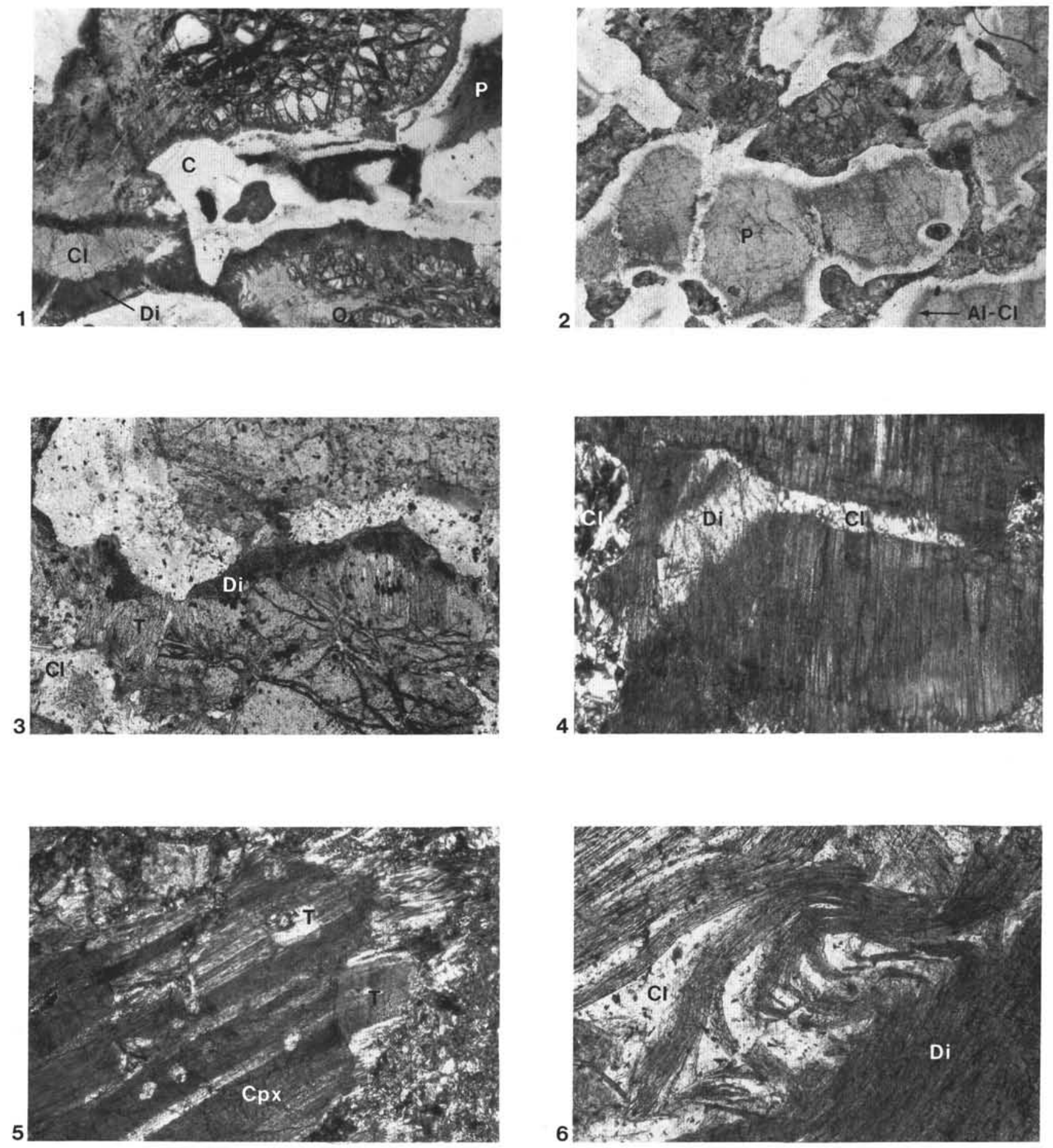

Plate 2. Photomicrographs of reaction and deformation microtextures in Site 895 mafic rocks. The width of Figures 1, 2, 4, and 5 represents 3.6 mm, whereas the width of Figures 3 and 6 represents $1.4 \mathrm{~mm}$. 1. Troctolite Sample 147-895D-7R-1 (Piece 13,81-84 cm). Typical coronas of colorless chlorite (C) between plagioclase $(\mathrm{P})$ and olivine $(\mathrm{O})$. Pseudomorphic replacement of olivine characterized by diopsidic (clinopyroxene \pm tremolite) rims (Di) at contact to chlorite corona, grading into tremolite and light green chlorite $(\mathrm{Cl})$ and mesh serpentine + magnetite (at top of photograph). Plagioclase replacement by dusty brown calc-silicate phases. Discontinuous microveins of diopsidic clinopyroxene crosscut pervasively altered olivine grains. 2. Moderately altered olivine gabbro Sample 147-895E-1 (Piece 9, 83-87 cm) with colorless chlorite forming coronas and filling cracks in relatively fresh plagioclase (P). Light green, Al-rich chlorite $(\mathrm{Al}-\mathrm{Cl})$ occurs at the rim of plagioclase relicts. 3. Close-up of zone pseudomorphic replacement textures of olivine, with clinopyroxene rims (Di) and radiating tremolite $(\mathrm{T})$ and chlorite $(\mathrm{Cl}) .4$. Highly altered coarse-grained diallage gabbro Sample 147-895D-8R-1 (Piece 8, 52-59 cm). Secondary diopsidic clinopyroxene and chlorite fills pull-apart cracks and replaces grain boundaries in kinked primary clinopyroxene. 5. Pervasively altered, coarse-grained diallage gabbro Sample 147-895E-4R-3 (Piece 4, 29-31 cm). Tremolite replaces kinked primary clinopyroxene (Cpx) along partings, in patches and along grain boundaries. 6. Pervasively altered, coarse-grained diallage gabbro Sample 147-895E-5R-1 (Piece 11, 56-60 cm). Deformation has resulted in microfolding of displaced clinopyroxene lamellae along $\{100\}$ partings filled by colorless chlorite. 

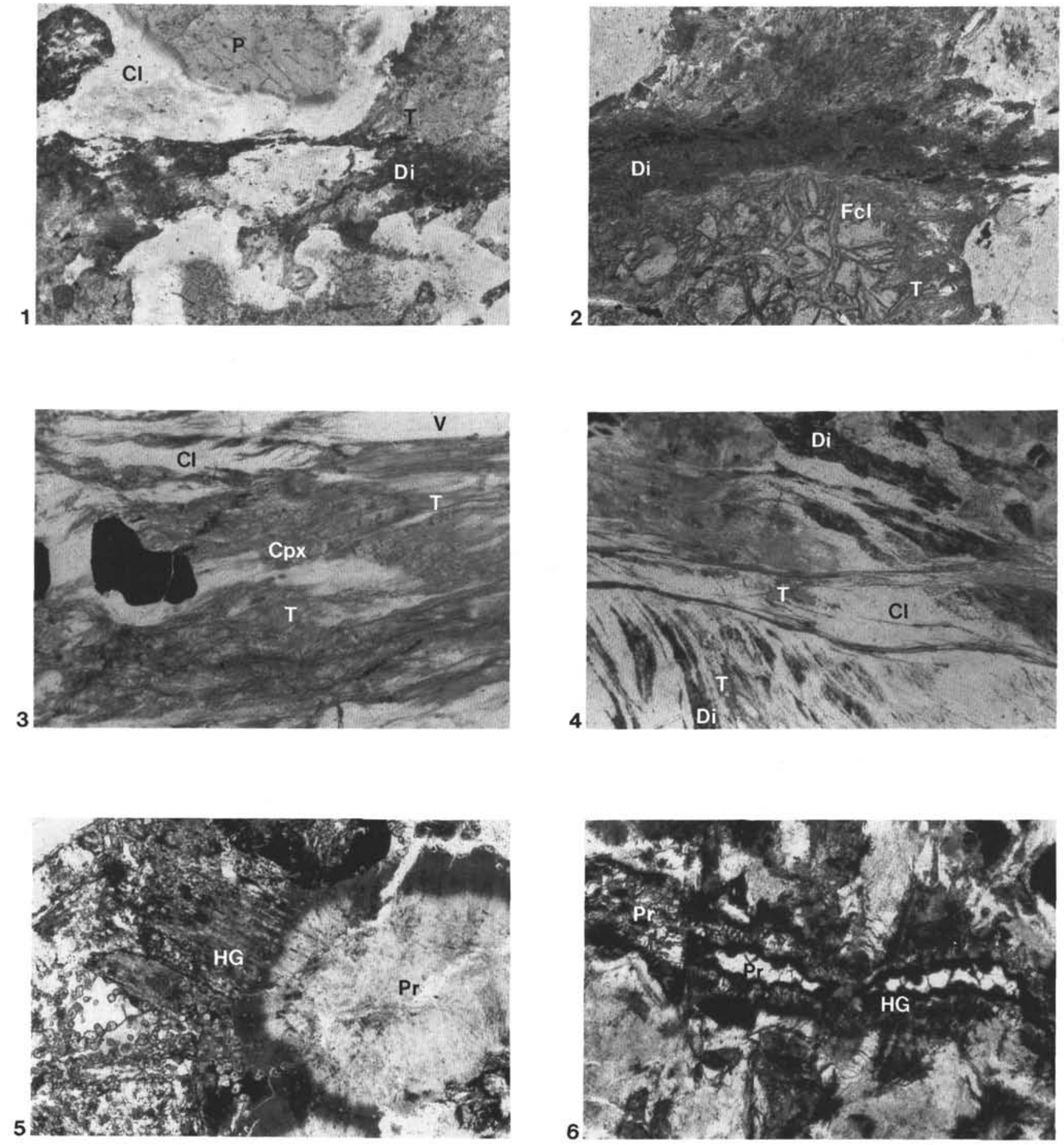

Plate 3. Deformation microfabrics in Site 895 gabbroic rocks. The width of Figures 1 and 3 through 6 represents 3.6 mm, whereas the width of Figure 2 represents $1.4 \mathrm{~mm} .1$ and 2. Localized microshear zone in Sample 147-895E-1R-1 (Piece 9, 83-87 cm). Penetrative deformation characterized by the alignment of chlorite $(\mathrm{Cl})+$ tremolite $(\mathrm{T})+$ diopsidic clinopyroxene $(\mathrm{Di})$ after olivine and new growth of clinopyroxene. Directly near the shear zone olivine is replaced by fibrous tremolite and yellow-green, pleochroic (Fe-rich) chlorite (Fcl). 3. Sheared metagabbro Sample 147-895E-6R-1 (Piece 14, 78-80 cm) with foliation defined by clinopyroxene $(\mathrm{Cpx})$; replaced by light brown, weakly pleochroic tremolitic amphibole $(\mathrm{T})$, transparent tremolite and chlorite $(\mathrm{Cl})$ with anomalous blue-violet interference colors. Black spinel grains are broken and have pressure shadows of chlorite. Foliation crosscuts and deforms a 4-mm-thick chlorite + diopside vein (V), indicating that high-strain deformation post-dated an earlier veining event. 4. In Sample 147-895D-10R-1 (Piece 8, 63-70 cm), light green chlorite with stringers of tremolite and secondary oxides form millimeter-thick shear bands, separated by highly elongated thinner bands of turbid, brownish tremolite and secondary oxides, 5. and 6. Characteristic boytrioidal texture of prehnite ( $\mathrm{Pr})$ and hydrogrossular (HG) veins. In Figure 5, prehnite forms clear, radiating blades in the centers of spherulites with reddish brown hydrogrossular phases along the rims (Sample 147-895E-2R-1, Piece 1, 91-125 cm). Finegrained aggregates of isotropic, granular hydrogrossular is associated with the replacement of plagioclase. In Figure 6, prehnite forms blocky crystals in the center of the vein with dark anhedral hydrogrossular + clay ( \pm oxides?) along the rims (Sample 147-895E-4R-2, Piece 17, 139-141 cm). 